\title{
REGULARITY AND STABILITY OF EQUILIBRIA IN AN OVERLAPPING GENERATIONS GROWTH MODEL
}

\author{
JEAN-FRANÇOIS MERTENS ${ }^{\dagger}$ AND ANNA RUBINCHIK ${ }^{\ddagger}$
}

\begin{abstract}
In an exogenous-growth economy with overlapping generations we analyse local stability of a balanced growth equilibrium (BGE) with respect to changes in consumption endowments, which could be interpreted as a transfer policy. We show that generically, in the space of parameters, equilibria around $\mathrm{BGE}$ are locally unique and are locally differentiable functions of endowments, with derivatives given by kernels. Further, those equilibria are stable in the sense that the effects of temporary changes decay exponentially towards $\pm \infty$. J.E.L. Classification numbers. D50, H43.
\end{abstract}

Date: January 21, 2014

Key words and phrases. Regularity of Infinite Economies, Policy Evaluation, Overlapping Generations, Exogenous Growth.

We would like to thank for its hospitality the Center for Rationality in Jerusalem, for many useful references A. Gorokhovsky, and for their comments K. Arrow, Cl. d'Aspremont, and participants of PET'08, SED'08, NBER General Equilibrium Conference in Iowa and Economic Theory Conference in Lawrence, KS, European General Equilibrium Conference at Warwick, Conference in honour of E. Kalai in Jerusalem, as well as the seminar participants at Boulder, Brussels, Cornell, Haifa, Northwestern, Roma, Salerno, Stony-Brook, UPenn, Yale and the "Séminaire de Jeux" in Paris.

This paper presents research results of the Belgian Program on Interuniversity Pôles of Attraction initiated by the Belgian State, Prime Minister's Office, Science Policy Programming. The scientific responsibility is assumed by the authors.

${ }^{\dagger}$ CORE, Université Catholique de Louvain; 34, Voie du Roman-Pays; B-1348 Louvain-la-Neuve; Belgique.

${ }^{\ddagger}$ Corresponding author. Dept. of Economics; University of Haifa; Mount Carmel, Haifa, 31905; Israel. E-mail: arubinchik@econ.haifa.ac.il. 


\section{Contents}

1. Introduction 2

1.1. Motivation and some related literature 2

1.2. A guide for an impatient reader 4

2. The model 6

$\begin{array}{ll}3 . & \text { The equilibrium system }\end{array}$

$\begin{array}{lll}\text { 3.1. Basic definitions } & 7\end{array}$

3.2. Fixed points of $\Upsilon$ (solutions) and equilibrium characterisation 8

4. Basic Tools: Implicit Function theorem and Wiener's theorem 10

4.1. Banach families and "smoothness", $S^{1} \quad 10$

4.2. The spaces $L_{p, q}^{\lambda}$ and Wiener's theorem 11

5. $\Upsilon$ is $S^{1} \quad 13$

6. Generic invertibility of $\frac{\partial F}{\partial k}$ at a BGE 19

6.1. BGE and the equilibrium graph 19

6.2. Derivative of the equilibrium map $\frac{\partial \Upsilon}{\partial k}$ at a BGE 21

6.3. Generic invertibility 23

6.3.1. Illustrating spectra of $\frac{\partial \Upsilon}{\partial k}$ at a BGE $\quad 23$

6.3.2. $\frac{\partial F}{\partial k}$ is generically invertible at a BGE 24

$\begin{array}{ll}7 . & \text { Speed of convergence }\end{array}$

7.1. Establishing the speed of convergence $\quad 29$

7.2. Illustrating the speed of convergence 30

8. Using the implicit function theorem 31

9. Derivatives are given by kernels 33

9.1. Kernel representation of $\left(\frac{\partial F}{\partial k}\right)^{-1}$ at a BGE 33

9.2. Kernel representations of the derivatives of $\varpi \quad 35$

10. Main Results 37

10.1. Regularity and stability of solutions $\quad 37$

10.2. From solutions to equilibria 39

10.3. Technical remarks (on the choice of the underlying spaces). 39

11. Conclusions 40

Appendix A. Auxiliary statements: concepts and tools 41

A.1. Kernels 41

A.2. Elementary operations 43

A.3. Composition with $C^{1}$ functions on $L_{\infty} \quad 44$

A.4. Simpler norms for $\mathbb{L}_{\Lambda}\left(C_{\infty}, C_{\infty}\right)$ and $\mathbb{L}_{\Lambda}\left(L_{\infty, 1}, C_{\infty}\right)$. 45

A.5. The weak topologies $\quad 46$

Appendix B. Discontinuity, in terms of $\lambda$, of the inversion 47

Appendix C. Zeros of a multivariate analytic function 47

References $\quad 48$

\section{INTRODUCTION}

1.1. Motivation and some related literature. Whether one is to analyse a pension reform, a change in the social security system, or an environmental project with a life longer than that of a human, one has to apply a model that distinguishes between generations and does not rely too heavily on an (at times convenient) assumption that individual lives have infinite span. Policy analysis in overlapping generations models brings new insights that are lacking in a static or a "representative" long-lived agents models, [7], [10]. The basic variant of such models was 
introduced in [1], [22], see [7], [15] for an overview. Working with overlapping generations models has a cost however, since they are prone to multiplicity (possibly continua) of equilibria ([14, 11], even in the presence of capital accumulation [21]), therefore potentially invalidating 'comparative dynamics' exercises.

Our main goal is to provide a first step to enable 'comparative dynamics' in an overlapping generations model: we show it is possible when time is taken as a real line (as opposed to the models where indeterminacy results are established - there time proceeds in discrete jumps and is being truncated at 0). A particular variant of such a model comes back to [3], and has experienced some recent development $([6],[5])$. Probably, the closest contribution (based on the defined objectives) is by Burke [2], who considers a discrete-time, but "eternal" economy and establishes determinacy of equilibria, in contrast to the previous literature [14]. Burke observes that only in a world where time is not truncated can one model truly perfect foresight. Fully anticipated policies in such a model can lead to a predictable "smooth" change in equilibrium. More "good news" in this respect appears in [8] demonstrating that indeterminacy (as in [14]) disappears in an exchange model (with no production) when time is extended infinitely far into the past and the gap in time between transactions tends to zero.

This is the main reason we work with a continuous time (taken as $\mathbb{R}$ ) overlapping generations model to perform a classical (à la Debreu) comparative statics exercise asking when one should expect a smooth response of equilibrium variables to a "small" change in endowment perturbations, interpreted as a policy (transfers of consumption goods across individuals, including a net transfer in the economy). In contrast to the existing literature on overlapping generations, we do not seek to describe the equilibrium system as a dynamic system (with a finite memory), rather, we view equilibrium variables as elements of a vector space, very much like we traditionally do in a finite economy. The advantage is the ability to analyse policy changes enacted over some interval of time, as opposed to just one-period changes ("impulses") traditionally dealt with in dynamic systems, and to identify the speed of convergence of the perturbed equilibrium to the status-quo (which should eventually yield differentiability of welfare). However, this requires some ground work, i.e., describing the spaces hosting the policy changes and the resulting equilibrium responses, done in [20], as well as characterising equilibria of the model, done in [19].

Known analysis of regularity of infinite economies (local uniqueness and differentiability of the equilibria) with a compact set of commodities or a finite number of consumers $[4,23]^{1}$ is based on extensions of Sard's theorem, that are not applicable here. Instead, we use Wiener's theorem on the spectrum of convolution operators to assure generic invertibility of the derivative of the equilibrium map required by the implicit function theorem. The new approach should help to identify a tractable way to verify regularity for a wide class of infinite economies.

The most exciting part of the new approach is the ability to evaluate analytically the first-order (approximated) response of the equilibrium variables to the policy change, i.e., we provide a way to calculate it. Although we work with a fully parameterised (yet classical) model, the analysis is broken into very small steps and each is generalised to the extent possible within the scope of a single paper. All the tools presented here can be used for a wide range of other models except for our proof of genericity that relies on a specific 'trick': yet even that might shed light on a way to generalise.

Let us stress that the approach we offer is in no way competing with a numerical one, rather, it might appear to be complementary. Verging to find an equilibrium

\footnotetext{
${ }^{1}$ Shannon and Zame [23] also provide a detailed overview of the literature.
} 
corresponding to a change in policy might be futile in the absence of any regularity results, especially with indeterminacy lurking. But even when regularity is established, constructing a good algorithm to find a new equilibrium is a tremendous challenge, since the map that has to be at its fixed point in equilibrium is not necessarily a contraction, and so starting with a guess for, say, a capital path in the vicinity of an equilibrium and applying the map recursively might lead away from the equilibrium. On the other hand, some of the steps in the approach we suggest can potentially be done using numerical methods.

1.2. A guide for an impatient reader. We start in section 2 by briefly describing the overlapping generations model from [19]. Borrowed from there also is the characterisation of its equilibria, interior with respect to ir-reversibility constraints, ${ }^{2}$ presented in section 3, where our task is formulated.

An exogenous (policy) variable is an endowment $E:(t, s) \mapsto E_{t, s}$, a locally integrable function of two real variables specifying (normalised) endowment transfers at time $t$ to an individual who is of age $s$ at that time. Aggregate endowment at any point in time is not restricted to be zero, it can have either sign. Given $E$, an equilibrium capital path (in terms of productive labour units), $k$ should satisfy the system

$$
F(k, E) \stackrel{\text { def }}{=} \Upsilon(k, E)-k=0
$$

where $\Upsilon$ is defined in prop. $1 .^{3}$ The status quo is a BGE (an equilibrium where capital is growing exponentially or $k$ is constant) and consumers have no endowment of consumption goods $(E=0)$, i.e., a manna-free BGE. We denote by $\varpi$ an equilibrium selection, a profile of equilibrium capital, output, consumption, interest rate and investment paths.

The main results, in section 10, provide regularity (existence, uniqueness, differentiability) and stability properties of $\varpi$ : it can be viewed then as an implicit function of $E$ in the neighbourhood of zero, returning values close to the original BGE (the status-quo). In addition, it is also differentiable in that neighbourhood. In particular, this implies that one can calculate the derivative of, say, capital with respect to the endowment perturbation at a given $\mathrm{BGE}$

$$
\varpi_{k}^{\prime}=-\left(\frac{\partial F}{\partial k}\right)^{-1} \circ \frac{\partial F}{\partial E}
$$

We also show that in that neighbourhood the derivatives of all equilibrium variables with respect to an exogenous $E$ are, basically, given by kernels (as functions of three real variables: $x, t, u(=t-s)$ ) which, roughly, measure the first-order change in the equilibrium variable at a given time $(x)$ as a result of the "shock" (a unit mass) at time $t$ in the exogenous variable $(E)$ for the generation of individuals who are of age $s$ at $t$. Based on the latter one can construct an algorithm to explicitly calculate the "impulse response" of all equilibrium variables.

The argument proceeds in several steps.

Step 1. Differentiability of $\Upsilon$ and hence of $F$.

The result is stated in lemma 1 in section 5 using the notion of differentiability from section 4, which relies on the tools developed in [20]. The stronger notion of

\footnotetext{
${ }^{2}$ With three potentially different physical goods: output, consumption, investment, while we assume that output can always be transformed into investment and consumption, the opposite transformation might or might not be feasible.

${ }^{3}$ For a solution of the fixed point system to be an equilibrium, it has to satisfy additional inequalities (irreversibility constraints and positivity of consumption), but let us focus on the solutions for this short introduction.
} 
differentiability we us allows to prove both regularity and stability using a single (implicit function) theorem.

Step 2. Invertibility of $\frac{\partial F}{\partial k}$ at a $B G E$.

The result is established in prop. 3 in section 6 . At first we show (lemma 2) that the kernel of the derivative of $\Upsilon$ with respect to $k$ at a BGE is a convolution kernel (roughly: the magnitude of the first-order effect of a perturbation at a BGE depends only on the distance in time from the perturbation and so is time-invariant). The result is based on lemma 1 containing the general form of the derivatives (at any interior equilibrium) and the characterisation of BGE (cor. 3).

Then, as a second step we can use Wiener's theorem (its extension is prop. 2) to get the generic (in the space of parameters) invertibility of the derivative: this amounts to verifying that 1 (generically) is not in the range of the Fourier transform of the (convolution) kernel of the derivative of $\Upsilon$ with respect to $k$ at a BGE (prop. 3 ). The same statement also provides a way to compute the Fourier transform of the inverse of the derivative $\frac{\partial F}{\partial k}$ at a BGE. Some heuristics for the result are given in section 6.1.

Step 3. The speed (exponential rates) of convergence.

The kernel of the derivative $\frac{\partial \Upsilon}{\partial k}$ at a BGE appears to be integrable even when multiplied by an exponential $\left(e^{\lambda t}\right)$ for $\lambda$ in an interval $\Lambda$ containing zero. This interval can be computed from cor. 4 , in section 7 , as another application of Wiener's theorem, prop. 2 .

In section 7.2 we compute interval $\Lambda$ for a few BGE in a range of economies and plot them in figures 13-20. The main result (in section 10) implies that such $\Lambda$ also determines the highest rates of exponential convergence of the equilibrium variables back to the status-quo in both directions in time.

Step 4. Derivatives are (mainly) given by kernels.

Whenever the implicit function theorem (lemma 3 in sect. 8 , based on $[20$, thm.3]) applies, the derivatives of all equilibrium variables (but investment) are given by kernels, as is shown in lemma 5 in section 9 . For investment, it is a kernel and a "shock" at the time when the aggregate endowment is changed, so that if the policy is a "pure transfer" (all transfers sum up to zero at any point in time), all derivatives are given just by kernels.

Step 5. Main result: regularity and stability.

In section 10 we formulate the regularity and stability results first for solutions of the fixed point, $\Upsilon(k, E)=k$, thm. 1 and then impose additional sufficient conditions for the results to apply to equilibria, thm. 2 .

Perhaps the most surprising result is the (uniform over $\Lambda$ ) Lipschitz property of the derivatives, thm. 1.iia, yielding exponential convergence of two non-BGE equilibria in the neighbourhood of a BGE to each other (thm.1.iic), i.e., the stability result in its strong form.

\section{THE MODEL}

For the analysis we use the general variant of the model in [19] (with CobbDouglas production) and the characterisation of the competitive equilibrium there.

The life span of any individual born at $x \in \mathbb{R}$ is $[0,1]$;

$$
U_{x}=\int_{0}^{1} e^{-\beta s} u\left(\hat{c}_{x, s}\right) d s, \text { with } u(z)=\frac{z^{1-\frac{1}{\sigma}}}{1-\frac{1}{\sigma}}, \text { for } \sigma \neq 1,{ }^{4}
$$

\footnotetext{
${ }^{4} u$ is extended by continuity to $[0,+\infty]$
} 
is his life-time utility defined over the set of individual consumption plans $\hat{c}_{x, s}$, $\overline{\mathbb{R}}_{+}$-valued Lebesgue-measurable functions of age $s$ for every $x$.

Individual derives income from renting labour and receiving transfers. ${ }^{5}$ Efficiency of labour varies with age according to a non-null integrable function $\zeta_{s} \geq 0$ over the life-span, $[0,1]$, and zero elsewhere. His time sells for $\int_{0}^{1} w_{x+s} \zeta_{s} d s$, where $x$ is his birth-date and $w_{t}$ is the (per unit-efficiency) wage rate at time $t$. His initial endowment of consumption goods is $\omega_{x, s}$ at age $s, \omega$ is locally integrable. ${ }^{6}$ So, with the price of consumption goods denoted by $p^{C}$, the individual's lifetime wealth is the value of his endowment (of consumption goods and of leisure),

$$
M_{x} \stackrel{\text { def }}{=} \int_{0}^{1}\left(p_{x+s}^{C} \omega_{x, s}+w_{x+s} \zeta_{s}\right) d s
$$

provided that integral is well-defined. ${ }^{7}$ In the baseline (status-quo) equilibrium the endowment is null.

The instantaneous production set is a subset of $\mathbb{R}^{5}$ describing feasible transformations of contracted productive labour $L_{t}$, capital $K_{t}$, investment $I_{t}$, consumption $C_{t}$ and an intermediate good $Y_{t}$ called 'output', produced using a Cobb-Douglas technology

$$
Y_{t}=A K_{t}^{\alpha} L_{t}^{1-\alpha}, 0<\alpha<1, A>0
$$

Aggregate total productive labour available at $t$ is:

$$
L_{t}=N_{0} e^{\gamma t} \int_{t-1}^{t} \zeta_{t-x} e^{\nu x} d x=N_{0} e^{(\gamma+\nu) t} \int_{0}^{1} \zeta_{s} e^{-\nu s} d s
$$

where $N_{x} d x \stackrel{\text { def }}{=} N_{0} e^{\nu x} d x\left(N_{0}>0\right)$ individuals get born in $[x, x+d x], \forall x \in \mathbb{R}, \nu$ is the rate of the population growth and $\gamma$ is the per-capita productivity growth. Aggregate capital evolves according to the differential equation ${ }^{8} K_{t}^{\prime}=I_{t}-\delta K_{t}$ with depreciation factor $\delta>0$ and is subject to

Assumption 1 (Initial Condition). For any feasible $K, e^{\delta t} K_{t}$ converges exponentially to 0 at $-\infty$.

The production set is any closed convex cone with free-disposal, containing the graph of the production function and the activities of transforming output into consumption and investment, and contained in the closed convex cone spanned by the production function, free-disposal, and 2-way transformations of output into consumption and investment.

Perfectly competitive firms have finite lives, for profits to be well-defined. Price of output, $p_{t}^{Y}$, is assumed to be finite. Labour $L_{t}$ is bought from individuals at price $w_{t}$, and capital $K_{t}$ is rented from investment firms at rate $r_{t} . C_{t}$ is sold at price $p_{t}^{C}$ to individuals and $I_{t}$ at price $p_{t}^{I}$ to investment firms. ${ }^{9}$

\footnotetext{
${ }^{5}$ The profits will be null due to constant-returns-to-scale production, defined below.

${ }^{6} \mathrm{~A}$ function is locally integrable on a topological space if every point has a measurable neighbourhood where the function is integrable.

${ }^{7}$ Since for any individual consumption bundle $c$ any equivalent function (coinciding with $c$ a.e.) has the same utility and the same budget, so we will think of it as an equivalence class of $\overline{\mathbb{R}}_{+}$-valued measurable functions. The same applies to all flows, $Y_{t}, I_{t}, C_{t}$, labour- and capital-services, and to their prices $p^{Y}, p^{I}, p^{C}, w$, and $r$. On the other hand, capital is a stock, so it and its price $p_{t}$ are defined pointwise, and no measurability restriction has a reason to apply. By the usual convention in measure theory, define any product of prices and quantities as 0 in case of a product $0 \times \infty$ or $\infty \times 0$. This allows to think of either prices or quantities as measures.

${ }^{8} \mathrm{In}$ its integral form the equation is $K_{t}=e^{-\delta\left(t-t_{0}\right)} K_{t_{0}}+\int_{t_{0}}^{t} e^{-\delta(t-s)} I_{s} d s$, where the integral is a Lebesgue integral (without loss of generality in this model, cf. [19, cor. 2]).

${ }^{9}$ For detailed description of the technology available to investment firms and the appropriate definition of their profits see [19, sect. 2.2.3].
} 
Notation 2.1. $\eta=(\gamma+\nu)(1-\sigma)+\beta \sigma, R=\gamma+\nu+\delta$. Intensive variables: $k_{t}=\frac{K_{t}}{L_{t}}$, $y_{t}=\frac{Y_{t}}{L_{t}}, i_{t}=\frac{I_{t}}{L_{t}}, c_{t}=\frac{C_{t}}{L_{t}}, \varphi_{s}=\frac{e^{-\nu s} \zeta_{s}}{\int_{0}^{1} e^{-\nu u} \zeta_{u} d u}, E_{t, s}=\frac{N_{t-s} \omega_{t-s, s}}{L_{t}},{ }^{10} \Omega_{t}=\int_{0}^{1} E_{t, s} d s$.

\section{The EQUILIBRIUM SYSTEM}

3.1. Basic definitions. A competitive equilibrium is defined in the classical way. Its characterisation, derived in [19], implies that, first, it is a solution of the equilibrium system, or a fixed point of the map defined below as $\Upsilon$, and, second, it satisfies inequalities, which assure that the solution yields non-negative consumption and does not violate (possible) irreversibility constraints imposed by technology (hence $0<i_{t}<y_{t}$ a.e.). Here we focus on two types of equilibria. The first is the statusquo, prevailing in the absence of a "policy intervention", so that $E=0$ (cf. notation 2.1) there. We assume it is a balanced growth equilibrium, i.e., an equilibrium where capital grows exponentially. The second is a (possibly non-stationary) equilibrium that emerges as a result of a perturbation of consumption endowments, or transfers, $E$, we will refer to it as a perturbed equilibrium.

Recall, our task is to represent an equilibrium (in the vicinity of the status-quo) as a smooth map $(\varpi)$ from the space of exogenous policy variable, $E$, into the spaces of equilibrium quantities and prices. Naturally, equilibrium conditions as well as assumptions imposed on the exogenous variable, $E$, determine the spaces where the equilibrium variables belong. The transfers, $E$, specify the "amount" of consumption good given to (or taken from) a person who is of age $s$ at time $t$, hence it, itself, is a map $\mathbb{R} \times[0,1] \rightarrow \mathbb{R}$. Our very first take on this problem [17] was written assuming $E$ is uniformly bounded, which we found restrictive. To allow for a rich set of transfer policies and yet work with tractable spaces, we derived and compiled the relevant properties of the so-called amalgams in [20]. Here is the definition adapted for the analysis of this model.

Definition 1 (amalgams). $\quad$ (i) Given a relatively compact measurable subset $H$ of $\mathbb{R}^{n}$ with non-empty interior,

$L_{p, q} \stackrel{\text { def }}{=}\left\{f\right.$ measurable: $\left.\mathbb{R}^{n} \rightarrow \overline{\mathbb{R}} \mid\|f\|_{p, q} \stackrel{\text { def }}{=}\|x \mapsto\| \mathbb{1}_{x+H} f\left\|_{q}\right\|_{p}<\infty\right\}$

$\bmod$ null functions, for $1 \leq p, q \leq \infty$.

(ii) For $1 \leq p \leq \infty, C_{p}$ is the subspace of continuous functions in $L_{p, \infty}$.

For example, for $h: \mathbb{R} \rightarrow \mathbb{R}$, let $H=[0,1]$, then $\|h\|_{\infty, 1}=\sup _{x} \int_{x-1}^{x}|h(t)| d t$. The policy variable $E$ is defined on $\mathbb{R} \times[0,1]$ and so $\|E\|_{\infty, 1}=\sup _{x} \int_{x-1}^{x} \int_{0}^{1}\left|E_{t, s}\right| d s d t$.

Assumption 2. $\|E\|_{\infty, 1}<\infty$.

Note that the assumption does not require the transfers to be uniformly bounded nor even totally summable over time.

3.2. Fixed points of $\Upsilon$ (solutions) and equilibrium characterisation. Since the technology allows for irreversibility, the prices of consumption, investment and output are not necessarily equal in an equilibrium, however the equilibria that will be characterised for our analysis here ${ }^{11}$ are interior with respect to such constraints and so the physical good has the same price, $p_{t}$, at any $t$, in all of its forms.

Proposition 1. Given an endowment $E_{t, s} \in L_{\infty, 1}$, define $\Upsilon:(k, E) \mapsto \tilde{k}$, from $C_{\infty} \times L_{\infty, 1}$ to $C_{\infty}$ as the composition of:

(i) $k \mapsto y: y_{t}=A k_{t}^{\alpha}$

\footnotetext{
${ }^{10} E_{t, s}$ is the normalised [per unit of productive labour at time $t$ ] aggregate endowment at time $t$ of individuals of age $s$.

${ }^{11}$ For an extended characterisation see [19].
} 
(ii) $k \mapsto \mathfrak{r}: \mathfrak{r}_{t}=R-\alpha A k_{t}^{\alpha-1}\left(=R-\frac{\alpha y_{t}}{k_{t}},=\gamma+\nu+\frac{p_{t}^{\prime}}{p_{t}}\right)$

(iii) $(\mathfrak{r}, E) \mapsto \mathscr{N}_{1}: \mathscr{N}_{1, x}=\int_{0}^{1} e^{\int_{x}^{x+s} \mathfrak{r}_{t} d t} E_{x+s, s} d s$

(iv) $(y, \mathfrak{r}) \mapsto \mathscr{N}_{2}: \mathscr{N}_{2, x}=\int_{0}^{1} e^{\int_{x}^{x+s}} \mathfrak{r}_{t} d t \varphi_{s} y_{x+s} d s$

(v) $\left(\mathscr{N}_{1}, \mathscr{N}_{2}\right) \mapsto \mathscr{N}: \mathscr{N}=\mathscr{N}_{1}+(1-\alpha) \mathscr{N}_{2}, \mathscr{N} \geq 0$

(vi) $\mathfrak{r} \mapsto \mathscr{D}: \mathscr{D}_{x}=\int_{0}^{1} e^{-\eta s+(1-\sigma) \int_{x}^{x+s} \mathfrak{r}_{t} d t} d s$

(vii) $(\mathscr{N}, \mathscr{D}) \mapsto \mathscr{B}: \mathscr{B}=\frac{\mathscr{N}}{\mathscr{D}}, \mathscr{B}_{j}=\frac{\mathscr{N}_{j}}{\mathscr{D}}(j=1,2), \mathscr{B}=\mathscr{B}_{1}+(1-\alpha) \mathscr{B}_{2}$

(viii) $(\mathfrak{r}, \mathscr{B}) \mapsto c: c_{t}=\int_{0}^{1} e^{-\eta u-\sigma \int_{t-u}^{t} \mathfrak{r}_{s} d s} \mathscr{B}_{t-u} d u$

(ix) $(y, E, c) \mapsto i: i_{t}=y_{t}+\Omega_{t}-c_{t}$

(x) $i \mapsto \tilde{k}: \tilde{k}_{t}=e^{-R t} \int_{-\infty}^{t} e^{R s} i_{s} d s>0$.

The prices then can be computed as follows,

$$
\begin{aligned}
& p_{t}=p_{0} e^{\int_{0}^{t}\left(\delta-R+\mathfrak{r}_{s}\right) d s} \\
& r_{t}=p_{t}\left(R-\mathfrak{r}_{t}\right) \\
& w_{t}=(1-\alpha) e^{\gamma t} p_{t}
\end{aligned}
$$

The zeros of $F(k, E) \stackrel{\text { def }}{=} \Upsilon(k, E)-k$, i.e., the fixed points of $\Upsilon$ (with implied values for $y, i, c$, etc.) characterize

Interior Equilibria: all equilibria where $0<i_{t}<y_{t}$ a.e., provided the zeros satisfy $0<i_{t}<y_{t}$;

BGE: if $K_{t}$ is exponential, all BGE with $\omega=0$.

Proof. The characterisation of each of the equilibria is by $\left[19\right.$, cor. $12 . c$, e]. $k_{t}$ is uniformly bounded by [19, prop. 1.a], $i_{t} \in L_{\infty, 1}$ by [19, prop. 1.b] and hence by equation $\mathrm{x}$ here, $k$ is continuous, thus the range.

Note that the prices $p_{t}, r_{t}, w_{t}$ are determined fully by the rest of the variables, in fact, it is enough to know the (adjusted) rate of change of prices, $\mathfrak{r}_{t}$, to determine the three. Clearly, also none of the variables determined by the conditions $\mathrm{i}-\mathrm{x}$ in prop. 1, together with $F(k, E)=0$ is affected by the three prices. Hence we can easily drop the three from the list of the equilibrium variables to lighten the notation and the analysis.

By [19, thm. 3] the number of BGE is finite in this economy and we denote by $\varpi$ an equilibrium selection for a given $E$. So, first, at $E=0, \varpi$ has to return one of the BGE. More generally, we want to establish existence of solutions

$$
(k, y, c, \mathfrak{r}, i) \in P \stackrel{\text { def }}{=} C_{\infty}^{4} \times L_{\infty, 1}
$$

of the equation system $\mathrm{i}-\mathrm{x}$ in prop. 1 , together with $F(k, E)=0$, as functions of $E \in L_{\infty, 1}\left(\mathbb{R}^{2}\right)$ (in a neighbourhood of 0 ). By 'equation system' we mean all equations there, but excluding the inequality $\mathscr{N}_{x} \geq 0$ sub v.

Further, for a solution to be an equilibrium it has to satisfy, in addition, the inequality $\mathscr{N} \geq 0$, thus assuring that individual consumption is non-negative, ${ }^{12}$ and, for all interior equilibria, the constraints $0<i_{t}<y_{t}$, implying that capital is positive as required by sub $(\mathrm{x})$. For the BGE with zero transfers this condition is satisfied by [19, cor. 9].

We will say that a BGE is regular if $\varpi$ can be viewed as a "smooth" (in the sense defined below) map from an endowment $E$ in some open $\|\cdot\|_{\infty, 1}$ neighbourhood of zero to the corresponding perturbed equilibrium. It is stable if each perturbed equilibrium converges exponentially fast to the status-quo path.

A natural way to establish regularity is to use an implicit function theorem (IFT). Heuristically, since any equilibrium has to be a solution to the fixed point problem

\footnotetext{
$12_{\mathscr{B}}$ has the same sign as the equilibrium consumption of an individual at age $u$, born at $t-u$ (cf. [19, comment 21]), which is also the sign of $\mathscr{N}$ by sub (vii), since $\mathscr{D} \geq 0$, which follows from sub (vi).
} 
$F(k, E) \stackrel{\text { def }}{=} \Upsilon(k, E)-k=0$, if such solution is an equilibrium, the derivative (in the sense defined below) with respect to $E$ of, say, equilibrium capital path, has to be determined by an IFT:

$$
\varpi_{k}^{\prime}=-\left(\frac{\partial F}{\partial k}\right)^{-1} \circ \frac{\partial F}{\partial E}
$$

Hence, from here our task is two-fold: one is to prove that $F$ is "smooth" and second, show that $\frac{\partial F}{\partial k}$ is invertible. As we will see this last claim is true only for a generic economy in the following sense.

Definition 2. The parameter space, or the space of economies, is $\wp=\{(R, \alpha, \eta, \sigma, \varphi(d s)) \mid$ $\left.(R, \sigma) \in \mathbb{R}_{++}^{2}, \alpha \in\right] 0,1[, \varphi(d s) \in \Delta([0,1])\}$, with the weak*-topology on $\Delta([0,1])$, the probabilities on $[0,1]$.

Definition 3. A subset of $\wp$ is negligible if its section for any fixed probability distribution $\varphi(d s)$ in $\Delta([0,1])$ has Lebesgue measure 0 .

A subset is generic if its complement is contained in a countable union of closed negligible sets.

\section{Basic Tools: Implicit Function theorem and Wiener's theorem}

First key insight we offer is a way to establish regularity and stability with one (implicit function) theorem, IFT. The idea is to show that it holds "uniformly" over a family of spaces indexed by the exponential speed of convergence of the perturbed equilibrium to the status quo.

The general IFT for families of Banach spaces is formulated and proved in [20, thm. 3], here we provide just the basic definitions needed to proceed.

\subsection{Banach families and "smoothness", $S^{1}$.}

Notation 4.1. For Banach spaces $X$ and $Y, \mathbb{L}(X, Y)$ is the Banach space of continuous linear maps from $X$ to $Y$; so $\mathbb{L}(X, X)$ is the Banach algebra of operators on $X . X \times Y$ and $X \cap Y$ have by default the maximum norm.

Definition 4. Fix a pointed set $A$, i.e. a pair $\left(A, \alpha_{0}\right)$ with $\alpha_{0} \in A$. Fix also a class of subsets $\mathcal{A}$ of $A$ with $\alpha_{0} \in Z \forall Z \in \mathcal{A}$.

A Banach family $(B, \mathcal{A})$, or shortly $B$, is a Banach space $(B,\|\cdot\|)$ endowed with a collection of pseudo-norms $\left(\|\cdot\|_{\alpha}\right)_{\alpha \in A}$, s.t. $\|\cdot\|_{\alpha_{0}}=\|\cdot\|$, where we allow pseudonorms to take infinite values. $B_{\alpha}=\left\{x \in B \mid\|x\|_{\alpha}<\infty\right\}$.

For 2 Banach families $X$ and $Y$, and a linear map $\varphi$ from $X$ to $Y$, let $\|\varphi\|_{\alpha}=$ $\sup \left\{\|\varphi(x)\|_{\alpha} \mid\|x\|_{\alpha} \leq 1\right\}$, and, for $Z \in \mathcal{A},\|\varphi\|_{Z}=\sup _{\alpha \in Z}\|\varphi\|_{\alpha}$; and let $\mathbb{L}_{\mathcal{A}}(X, Y)=$ $\left\{\varphi \in \mathbb{L}(X, Y) \mid \forall Z \in \mathcal{A},\|\varphi\|_{Z}<\infty\right\}$, endowed with the family of norms $\left(\|\cdot\|_{Z}\right)_{Z \in \mathcal{A}}$.

Definition 5. For Banach families $(X, \mathcal{A})$ and $(Y, \mathcal{A})$, and $O$ open in $X, F: O \rightarrow Y$ is $S^{1}$ (or: $S_{\mathcal{A}}^{1}$ ) if $F$ is Gâteaux-differentiable at each $x \in O$, with derivative $F_{x}^{\prime} \in$ $\mathbb{L}_{\mathcal{A}}(X, Y)$, and if, $\forall\|\cdot\|_{Z}(Z \in \mathcal{A}), x \mapsto F_{x}^{\prime}$ is locally Lipschitz on $O$.

To index the Banach families by the power of the exponential, as mentioned at the beginning of the section, we introduce operator $\phi_{\lambda}$ :

Notation 4.2. For $\boldsymbol{\lambda} \in \mathbb{R}^{n}, \phi_{\lambda}$ is the multiplication operator by $e^{\langle\boldsymbol{\lambda}, t\rangle}$ on functions of $t \in \mathbb{R}^{n}$ to a vector space; i.e., $\phi_{\lambda}(f)=\left[t \mapsto e^{\langle\boldsymbol{\lambda}, t\rangle} f(t)\right] .{ }^{13}$ For our purposes it will be sufficient to consider just $\lambda=(\lambda, 0, \ldots, 0) \in \mathbb{R}^{n}$, with $\lambda \in \mathbb{R}$.

$L_{p}^{\lambda} \stackrel{\text { def }}{=} \phi_{-\lambda}\left(L_{p}\right)$, with $\|f\|_{p}^{\lambda} \stackrel{\text { def }}{=}\left\|\phi_{\lambda}(f)\right\|_{p}$. Similarly for $L_{p, q}$ and $C_{p}$.

\footnotetext{
${ }^{13}$ So $\boldsymbol{\lambda} \rightarrow \phi_{\lambda}$ is a group isomorphism.
} 
Recall that the equilibrium variables belong either to $L_{\infty, 1}$ or to $C_{\infty} \subset L_{\infty}$. Since the endowment perturbation, $E$ is defined on a subset of $\mathbb{R}^{2}$ (time and age), we will take $\mathbb{R}^{n}$ as domain for all. So, for our analysis here we consider the three Banach families defined below.

Definition 6. Fix the pointed set $\left(A, \alpha_{0}\right) \stackrel{\text { def }}{=}(\{(\lambda, p) \in \Lambda \times[1, \infty]\},(0, \infty))$, with $\Lambda$ open in $\mathbb{R}, 0 \in \Lambda$. Initially $\Lambda=\mathbb{R} . \mathcal{A} \stackrel{\text { def }}{=}\{J \times[1, \infty]\}$ where $J$ is a compact subinterval of $\Lambda$ with 0 interior. The 3 Banach spaces, $L_{\infty, 1}\left(\mathbb{R}^{n}\right), L_{\infty}\left(\mathbb{R}^{n}\right)$, and $C_{\infty}\left(\mathbb{R}^{n}\right)$ are endowed with $\left(\|\cdot\|_{p}^{\lambda}\right)_{\lambda \in \Lambda}$ as family, meaning $\|\cdot\|_{p, 1}^{\lambda}$ in the first case and $\|\cdot\|_{p, \infty}^{\lambda}$ else.

Observe that $\|\cdot\|_{\alpha_{0}}=\|\cdot\|$ in all 3 cases, ${ }^{14}$ as required by definition 4 .

Notation 4.3. $\mathbb{L}^{\Lambda}$ will stand more explicitly for the corresponding $\mathbb{L}_{\mathcal{A}}$, and $\|\cdot\|_{J}$ for $\|\cdot\|_{J \times[1, \infty]}$.

4.2. The spaces $L_{p, q}^{\lambda}$ and Wiener's theorem. Recall that in order to use IFT one has to verify invertibility of $\partial F / \partial k$ [cf. heuristic equation (1)]. So, now we have to learn how to invert linear operators defined on the families of spaces indexed by $\lambda$, which, first of all, requires to define the corresponding algebra.

Notation 4.4. Let $M$ be the set of bounded measures (on $\mathbb{R}^{n}$ ). $\phi_{\lambda}$ acts also on measures $\mu$ by $\left(\phi_{\lambda}(\mu)\right)(d t)=e^{\langle\lambda, t\rangle} \mu(d t)$.

For a function $X$ on (subset of) $\mathbb{R}^{n}$ let $\bar{X}(x)=X(-x)$. $\Re(z)$ denotes the real part and $\Im(z)$ the imaginary part of a complex number $z$.

Definition 7. For $f \geq 0$ Lebesgue-measurable and $\mu \in M_{+}$the convolution $\mu \star f$ is the equivalence class of $t \mapsto \int \tilde{f}(t-s) \mu(d s)$ for any $\tilde{f} \in f$.

For $\mu \in M$, its Fourier transform (FT) $\widehat{\mu}(\omega)=\int e^{\mathrm{i} \omega t} \mu(d t)\left(\widehat{g}\right.$ for $\left.g \in L_{1}\right)$.

Take 2 Radon measures $\mu$ and $\nu$ s.t. $|\mu \times \nu|(\{x, y \mid x+y \in K\})<\infty \forall K \in \mathcal{K}$. Define their convolution as the measure $\mu \star \nu: f \mapsto \int f(s+t) \mu(d s) \nu(d t)$ for every continuous function $f$ with compact support.

The Banach algebra (Wiener algebra) $\mathcal{W}$ is the subspace of $M$ spanned by $L_{1},{ }^{15}$ with multiplication given by convolution and the identity being $\epsilon_{0}$, the unit mass at $0 . \mathcal{W}^{\lambda}=\phi_{-\lambda}(\mathcal{W})$.

Remark 1. (i) As is shown in [20, thm.2.i], convolution turns $M$ into a commutative Banach algebra (of convolution operators) on $L_{p, q}$ and $C_{p}$ for $1 \leq p, q \leq \infty$.

(ii) $\widehat{\mu \star \nu}=\widehat{\mu} \widehat{\nu}$, so the FT is an injective algebra homomorphism of norm 1 from $M$ to $C_{\infty}$.

Finally, we can formulate the following proposition based on the Wiener's theorem that provides an explicit way to calculate the convolution inverse of $f \in L_{1}^{\lambda}$ (for $\lambda$ in an interval) in $\mathcal{W}^{\lambda}$. Note that the inverse is the same for all $\lambda$ in that interval.

Proposition 2. Let $f: \mathbb{R} \rightarrow \overline{\mathbb{R}}$ be Lebesgue-measurable and $J=\left\{\lambda \mid f \in L_{1}^{\lambda}\right\}$. Then $J$ is connected; denote its interior by $J^{\circ}$. Let, for $\Re(z) \in J, h(z)=\int e^{z t} f(t) d t$, so $h$ is analytic for $\Re(z) \in J^{\circ}$, and let $D=\{\Re(z) \in J \mid h(z)=1\}$. D is closed in $J$, and $D \cap J^{\circ}$ discrete. For any connected component $\Lambda$ of $J \backslash D, f-\mathbf{1}$ has a convolution inverse $g-\mathbf{1}$ in $\bigcap_{\Lambda} \mathcal{W}^{\lambda}$, and $\int e^{z t} g(t) d t=\bar{h}(z) \stackrel{\text { def }}{=} \frac{1}{1-\frac{1}{h(z)}}$ for $\Re(z) \in \Lambda$.

Remark 2. There can be several distinct sets $\Lambda$ with different convolution inverses $g-1$ in each (cor. 2), as illustrated in app. B (and by Figs. 13-20).

\footnotetext{
${ }^{14} L_{\infty, \infty}=L_{\infty}$ by [20, thm. 1.vi].

${ }^{15}$ So any function $f \in L_{1}$ is associated with a measure $f d \mu \in M$ for the Lebesgue measure $\mu$ on $\mathbb{R}^{n}$.
} 
Proof. $J$ is connected: for any $\lambda \in\left[\lambda_{1}, \lambda_{2}\right] e^{\lambda} \leq e^{\lambda_{1}}+e^{\lambda_{2}}$, so with $\lambda_{i} \in J$, $\int e^{\lambda t}|f(t)| d t \leq \int\left(e^{\lambda_{1} t}+e^{\lambda_{2} t}\right)|f(t)| d t<\infty$, so $f \in L_{1}^{\lambda}$.

Observe that $h(z)$ is analytic on $\Re(z) \in J^{\circ}$, since the integral under $\left|e^{z t} f(t)\right| d t$ of the power series of $e^{a t}$ converges absolutely for sufficiently small $|a|$.

Next we show that $h(\lambda+\mathrm{i} \omega)$ converges when $\omega \rightarrow \pm \infty$ uniformly to 0 for $\lambda$ in compact subsets of $J$. Indeed, this compact subset can be taken as an interval $\left[\lambda_{1}, \lambda_{2}\right]$; with $\varphi(t)=e^{\lambda_{1} t}+e^{\lambda_{2} t}$, approximate now $\varphi f$ up to $\varepsilon$ in $L_{1}$ by $\varphi \psi$, where $\psi$ is a linear combination of indicator functions of intervals: so it suffices to prove the claim when $f$ is such an indicator function, where it results e.g. by direct calculation.

The same proves also continuity of $h$, so $h$ is bounded on compact subsets of $J$.

By continuity, $S=\{z \mid \Re(z) \in J, h(z)=1\}$ is closed in $\{z \mid \Re(z) \in J\}$, and, by the above uniform convergence on compact subsets, the projection to $J$ is proper (compact sets have compact inverse images), so $D$ is closed in $J$. The analyticity of $h$ implies that $\left\{z \in S \mid \Re(z) \in J^{\circ}\right\}$ is discrete, thus so is $D \cap J^{\circ}$, again by properness.

By lemma $6 . \mathrm{i}$ (in app. A), to compute the inverse of $f-\mathbf{1}$ in $\mathcal{W}^{\lambda}$, map everything to $\mathcal{W}\left(=\mathcal{W}^{0}\right)$, and use there Wiener's theorem [13, thm. 32 p. 340], ${ }^{16}$ that, for $\phi_{\lambda}(f)=r \in L_{1}, \mathbf{1}-r$ is invertible in $\mathcal{W}$ iff $\widehat{r}$ does not take the value 1 (i.e., $1 \notin$ the closure of $\{\widehat{r}(\omega)\}$, since $\left.\widehat{r} \in C_{0}\right)$. Then the inverse must be of the form $\mathbf{1}-r^{\prime}$, with FT's $\widehat{r}$ and $\widehat{r}^{\prime}$ satisfying $\widehat{r}^{\prime}=\frac{1}{1-1 / \hat{r}}$; the inverse of $f-\mathbf{1}$ in $\mathcal{W}^{\lambda}$ is then $g_{\lambda}-\mathbf{1}$ with $g_{\lambda}=\phi_{-\lambda}\left(r^{\prime}\right)$.

By definition, $h(\lambda+\mathrm{i} \omega)=\widehat{\phi_{\lambda}(f)}(\omega)=\widehat{r}(\omega)$. So, $\mathbf{1}-r$ is invertible iff $h(z) \neq 1$ for $\Re(z)=\lambda$, with as inverse $\mathbf{1}-r^{\prime}$ where $\widehat{r}^{\prime}(\omega)=\bar{h}(\lambda+\mathrm{i} \omega)$. Now, since $g_{\lambda}=\phi_{-\lambda}\left(r^{\prime}\right)$, Parseval's formula [12, II.2] yields, for $\varphi \in K, \int \varphi(t) g_{\lambda}(t) d t=$ $\int \varphi(t) e^{-\lambda t} r^{\prime}(t) d t=\frac{1}{2 \pi} \int \bar{h}(\lambda+\mathrm{i} \omega)\left[\int \varphi(t) e^{-(\lambda+\mathrm{i} \omega) t} d t\right] d \omega$. The bracket is integrable in $\omega$ and $\bar{h}$ bounded. Let now $r_{T}^{\prime}(t)=\frac{1}{2 \pi} \int_{-T}^{T} e^{-(\lambda+\mathrm{i} \omega) t} \bar{h}(\lambda+\mathrm{i} \omega) d \omega$; then we get $\int \varphi(t) r_{T}^{\prime}(t) d t=\frac{1}{2 \pi} \int_{-T}^{T} \bar{h}(\lambda+\mathrm{i} \omega)\left[\int \varphi(t) e^{-(\lambda+\mathrm{i} \omega) t} d t\right] d \omega$; by the integrability of the integrand this converges for $T \rightarrow \infty$ to our previous formula for $\int \varphi(t) g_{\lambda}(t) d t$ : so $r_{T}^{\prime} \rightarrow g_{\lambda}$ in $K^{*}$.

For $\lambda_{1}, \lambda_{2} \in \Lambda \cap J^{\circ}$, the integrand in $r_{T}^{\prime}, e^{-z t} \bar{h}(z)$, is analytic for $\lambda_{1} \leq \Re(z) \leq \lambda_{2}$, so $r_{T}^{\prime}$ at $\lambda_{1}$ and $\lambda_{2}$ differs by $\int_{\lambda_{1}}^{\lambda_{2}} e^{-(x-\mathrm{i} T) t} \bar{h}(x-\mathrm{i} T) d x-\int_{\lambda_{1}}^{\lambda_{2}} e^{-(x+\mathrm{i} T) t} \bar{h}(x+\mathrm{i} T) d x$. $\bar{h}$ converges, as $h$, uniformly for $\lambda \in\left[\lambda_{1}, \lambda_{2}\right]$ to 0 when $\omega \rightarrow \infty$, so each of those 2 integrals is bounded in norm by $\left|\lambda_{1}-\lambda_{2}\right| e^{\max \left|\lambda_{1} t\right|,\left|\lambda_{2} t\right|} O(T)$. By the dominated convergence theorem, this bound for the difference of the $r_{T}^{\prime}$ remains valid $\forall \lambda_{1}, \lambda_{2} \in \Lambda$. It tends to 0 in $K^{*}$ as $T \rightarrow \infty$. So $g_{\lambda}$, the limit in $K^{*}$, is independent of $\lambda \in \Lambda$; call it $g$ : $g \in \bigcap_{\Lambda} L_{1}^{\lambda}$, and $r^{\prime}=\phi_{\lambda}(g), \widehat{r}^{\prime}(\omega)=\bar{h}(\lambda+\mathrm{i} \omega) \Rightarrow \int e^{z t} g(t) d t=\bar{h}(z)$ for $\Re(z) \in \Lambda$.

Corollary 1. For $\lambda \in J \backslash \Lambda, g \notin L_{1}^{\lambda}$.

Proof. Else let $\Lambda_{0}$ be the smallest interval containing $\lambda$ and $\Lambda: g \in \cap_{\Lambda_{0}} L_{1}^{\lambda}$, and $f$ too; so $(\mathbf{1}-f) \star(\mathbf{1}-g)=\mathbf{1}$ yields $(1-h)(1-\bar{h})=1$, which is impossible when $h(z)=1, \bar{h}$ being finite. Thus $\Lambda_{0} \cap D=\emptyset$, contradiction.

Corollary 2. The inverses $g$ in 2 different connected components $\Lambda$ differ.

Prop. 2 will be later applied to the kernel of the derivative of $\Upsilon$ with respect to $k$, see sect. 7 . Since the kernel depends solely on the primitives and the initial BGE chosen, so will be the interval $\Lambda$ and the main results will establish that this interval determines the exponential speed of convergence of equilibrium variables back to the status-quo as a result of a temporary policy change. Thus one can determine just how stable the equilibria are.

Moreover, in contrast to Sard's theorem (which is also not applicable here), Wiener's theorem (and hence prop. 2) gives us a way to explicitly calculate (the Fourier transform of) the inverse of $\partial F / \partial k$ at a BGE and hence the full equilibrium

\footnotetext{
${ }^{16}$ The theorem also states that $\mathcal{W}$ is inverse-closed in $\mathbb{L}\left(L_{p}, L_{p}\right)$.
} 
response, $\varpi_{k}^{\prime}$ using the IFT. Such calculation should be feasible since, as we show in sect. 9, the derivatives of $\Upsilon$ with respect to endogenous $k$ and exogenous $E$ are given by kernels and at a fixed point corresponding to a BGE they are convolution kernels (and Fourier transform of a convolution is easy to compute).

Now with the tools in place, we can move to the first item on the agenda: prove that $\Upsilon$ (and hence $F$ ) is smooth, i.e., it is $S^{1}$.

\section{5. $\Upsilon$ IS $S^{1}$}

Notation 5.1. For $\mathfrak{r}_{t}$ satisfying the equilibrium condition $\mathfrak{r}_{t}=R-\alpha A k_{t}^{\alpha-1}$,

$$
\begin{array}{ll}
X_{x, t} \stackrel{\text { def }}{=} \mathbb{1}_{0 \leq t-x \leq 1} e^{\int_{x}^{t} \mathfrak{r}_{s} d s} & Z_{x, t} \stackrel{\text { def }}{=} \mathbb{1}_{0 \leq t-x \leq 1} e^{\int_{x}^{t}\left(-\eta-\sigma \mathfrak{r}_{s}\right) d s} \\
\mathrm{k}_{E}^{c}(t ; x, u) \stackrel{\text { def }}{=} \frac{Z_{u, t} X_{u, x}}{\int X_{u, u+z} Z_{u, u+z} d z} & \xi_{R}(x) \stackrel{\text { def }}{=} \mathbb{1}_{x \geq 0} e^{-R x} \\
\mathrm{k}_{E}^{k d}(t, x) \stackrel{\text { def }}{=} \int \xi_{R}(t-z) \epsilon_{x}(z) d z, & \mathrm{k}_{k}^{k d}(t ; x) \stackrel{\text { def }}{=} \int \xi_{R}(t-z) \alpha \frac{y_{z}}{k_{z}} \epsilon_{x}(z) d z \\
\mathrm{k}_{E}^{k c}(t, x, u) \stackrel{\text { def }}{=} \int \xi_{R}(t-z) \mathrm{k}_{E}^{c}(z ; x, u) d z & \mathrm{k}_{k}^{k c}(t ; x) \stackrel{\text { def }}{=} \int \xi_{R}(t-z) \mathrm{k}_{k}^{c}(z ; x) d z \\
\mathrm{k}_{E}^{k}(t ; x, u) \stackrel{\text { def }}{=} \mathrm{k}_{E}^{k d}(t ; x)-\mathrm{k}_{E}^{k c}(t ; x, u) & \mathrm{k}_{k}^{k}(t ; x) \stackrel{\text { def }}{=} \mathrm{k}_{k}^{k d}(t ; x)-\mathrm{k}_{k}^{k c}(t ; x)
\end{array}
$$

Lemma 1. (i) For $E, i, \in L_{\infty, 1}, c, \mathfrak{r}, k, y \in C_{\infty}$, and $k_{t}$ : $\inf _{t} k_{t}>0$,

(a) $k \mapsto y$ is $S^{1}$ with derivative $\delta y_{t}=\alpha A k_{t}^{\alpha-1} \delta k_{t}$.

(b) $k \mapsto \mathfrak{r}$ is $S^{1}$ with $\delta \mathfrak{r}_{t}=\alpha(1-\alpha) A k_{t}^{\alpha-2} \delta k_{t}$. So $\delta \mathfrak{r}=(1-\alpha) \frac{\delta y}{k}$.

(c) $(k, E) \mapsto c$ is $S^{1}$ with derivative

$$
\delta c_{t}=\iint \mathrm{k}_{E}^{c}(t, x, x-s) \delta E_{x, s} d s d x+\int \mathrm{k}_{k}^{c}(t, z) \delta k_{z} d z
$$

for a bounded, continuous $\mathrm{k}_{k}^{c}(t, z)$, zero for $t-z \notin[-1,1]$;

$\mathrm{k}_{E}^{c}$ is bounded too.

It is even $S^{1}$ from $L_{\infty, 1}^{2}$ to $C_{\infty}$.

(d) $E \mapsto \Omega$ is $S^{1}$ with derivative $\delta \Omega_{t}=\int \delta E_{t, s} d s$

(e) $(y, E, c) \mapsto i$ is $S^{1}$ with derivative $\delta i_{t}=\delta y_{t}+\delta \Omega_{t}-\delta c_{t}$

(f) $i \mapsto \tilde{k}$ is $S^{1}$, with derivative $\delta \tilde{k}_{t}=\int_{0}^{\infty} e^{-R s} \delta i_{t-s} d s$.

(ii) $\Upsilon:(k, E) \rightarrow \tilde{k}$ is $S^{1}$ from $C_{\infty} \times L_{\infty, 1}$ to $C_{\infty}$ on $\left\{\right.$ inf $\left.k_{t}>0\right\}$.

(iii) $\frac{\partial \Upsilon}{\partial E}=\delta E \mapsto\left[t \curvearrowright \int \mathrm{k}_{E}^{k}(t ; x, x-s) \delta E_{x, s} d s d x\right]$

(iv) $\frac{\partial \Upsilon}{\partial k}=\delta k \mapsto\left[t \curvearrowright \int \mathrm{k}_{k}^{k}(t ; x) \delta k_{x} d x\right]$

(v) Both $\mathrm{k}_{k}^{k c}$ and $\mathrm{k}_{k}^{k d}$ are locally Lipschitz from $C_{\infty} \times L_{\infty, 1}$ to $\|\cdot\|_{J}^{L C} \forall J \subset \Lambda$, where $\|\mathrm{k}\|_{J}^{L C}=\sup _{x} \operatorname{ess~sup~}_{s, t} \psi_{J}(x-t)|\mathrm{k}(x ; t, u)|, \psi_{\lambda, \lambda^{\prime}}(z)=e^{\lambda z}+e^{\lambda^{\prime} z}$, and for a compact interval $J, \psi_{J}=\psi_{\min J, \max J} \cdot{ }^{17}$

(vi) $\mathrm{k}_{k}^{k c}(t ; x)$ is continuous and is Lipschitz in $t$ to $C_{\infty}$, and bounded by a constant times $\xi_{R}(t-x+1), \mathrm{k}_{k}^{k}$ is bounded.

(vii) $\mathrm{k}_{E}^{k c}(t, x, x-s)$ is Lipschitz on $s \in[0,1]$, and bounded by a constant times $\mathbb{1}_{s \in[0,1]} \xi_{R}(t-x+s)$.

(viii) For $\mu \in M, \mu \mapsto \varrho_{E}^{k}(\mu ; \cdot, \cdot) \stackrel{\text { def }}{=} \int \mathrm{k}_{E}^{k}(x ; t, u) \mu(d x)$ is continuous and bounded from bounded subsets of $\left(M, \sigma\left(M, C_{\infty}\right)\right)$ to $\left(L_{1, \infty}, \tau\left(L_{1, \infty}, L_{\infty, 1}\right)\right)$.

(ix) At non-atomic $\mu$, it is continuous from $M_{+}$to $L_{1, \infty}$, and with values in $C_{1}$. In particular, it is continuous from $L_{1}$ to $C_{1}$.

(x) For a measure space $(\Omega, \mathcal{A}, \nu)$ and $\mu(\omega)$ measurable s.t. $\|\mu(\omega)\| \in L_{1}(\nu)$, $\iint \varrho_{E}^{k}(\mu(\omega) ; t, t-s) f_{t, s} d s d t \nu(d \omega)=\int \varrho_{E}^{k}\left(\int \mu(\omega) \nu(d \omega) ; t, t-s\right) f_{t, s} d s d t \forall f \in$ $L_{\infty, 1}$. In particular, $\int \varrho_{E}^{k}(\mu ; t, t-s) f_{t, s} d s d t=\iint \varrho_{E}^{k}(x ; t, t-s) f_{t, s} d s d t \mu(d x)$.

(xi) The same conclusions hold for $\varrho_{E}^{k c}(\mu ; t, u) \stackrel{\text { def }}{=} \int \mathrm{k}_{E}^{k c}(x ; t, u) \mu(d x)$ and $\varrho_{E}^{k d}(\mu ; t, u) \stackrel{\text { def }}{=}$ $\int \mathrm{k}_{E}^{k d}(x ; t, u) \mu(d x)$ defined for $\mu \in M$.

(xii) $\varrho_{E}^{k c}(\mu, x, x-s)$ is continuous to $C_{1}$, and is, for some $K$ and $\forall \mu$, Lipschitz in $(x, s)$ with constant $K\|\mu\|$.

\footnotetext{
${ }^{17}$ A superscript like LC stands for "from $L_{\infty, 1}\left(\mathbb{R}^{2}\right)$ to $C_{\infty}$ ".
} 
Remark 3. If a uniformly continuous function $h$ on $\mathbb{R}^{n}$ is bounded by $\xi_{R} \circ \phi$, where $\phi$ is affine, then $e^{\lambda \phi(x)} h(x)$ is uniformly continuous $\forall \lambda<R$.

Proof. ia and ib: by lemma 16.

ic: First, let $\mathscr{N}_{0}=\mathscr{N}$. Then $(\mathfrak{r}, E, y) \mapsto \mathscr{N}_{i}$ is $S^{1}$ with derivative

$$
\delta \mathscr{N}_{i, x}=\int\left[H_{i}(x, z) \delta \mathfrak{r}_{z}+h_{i}\left(x, z, \delta y_{z}, \delta E_{z, z-x}\right)\right] d z \quad \text { where }
$$

$h_{1}(x, s, u, v)=v X_{x, s}, h_{2}(x, s, u, v)=h_{1}\left(x, s, 0, u \varphi_{s-x}\right), h_{0}=h_{1}+(1-\alpha) h_{2}$,

$H_{i}(x, z)=\mathbb{1}_{x \leq z} \int_{z}^{\infty} h_{i}\left(x, s, y_{s}, E_{s, s-x}\right) d s$.

Indeed, apply lemma 18 , with $g_{t}=\mathfrak{r}_{t}, \vartheta=\mathbb{1}_{[0,1]}$, and $h_{t, s}=E_{t, s}+(1-\alpha) y_{t} \varphi_{s}$, and cor. 11 for $y \mapsto y \varphi$.

Further, $(\mathfrak{r}, E) \mapsto \mathscr{N}_{1}$ is even $S^{1}$ from $L_{\infty, 1}^{2}$ to $L_{\infty, 1}$, and $(\mathfrak{r}, y) \mapsto \mathscr{N}_{2}$ from $L_{\infty, 1}^{2}$ to $L_{\infty, 1}$ and from $L_{\infty, 1} \times C_{\infty}$ to $C_{\infty}$. Indeed, use lemma 17 with $\vartheta=\mathbb{1}_{[0,1]}$ for the exponential term, then lemma 10 implies that $(\mathfrak{r}, y) \mapsto \mathbb{1}_{[0,1]}(s) e^{\int_{x}^{x+s} \mathfrak{r}_{t} d t} y_{x+s}$ is $S^{1}$ from $L_{\infty, 1} \times L_{\infty}$ to $L_{\infty}$, so, continuity being obvious, from $L_{\infty, 1} \times C_{\infty}$ to $C_{\infty}(\mathbb{R} \times[0,1])$. Use then lemma 12 .

Next, $\mathfrak{r} \mapsto \mathscr{D}$ is $S^{1}$ with derivative

$$
\delta \mathscr{D}_{x}=(1-\sigma) \int_{x}^{x+1} \delta \mathfrak{r}_{s} \int_{s}^{x+1} X_{x, z} Z_{x, z} d z d s
$$

This follows from lemma 19 , setting $g_{t}=(1-\sigma) \mathfrak{r}_{t}-\eta, \vartheta=\mathbb{1}_{[0,1]}$ and $z_{t}=1$. It is even $S^{1}$ from $L_{\infty, 1}$ to $C_{\infty}$.

Next, $\mathscr{D} \mapsto \frac{1}{\mathscr{D}}$ is $S^{1}$ by lemma 16 , so by lemmas 9 and $10(\mathscr{N}, \mathscr{D}) \mapsto \mathscr{B}$ is $S^{1}$ with derivative $\delta \mathscr{B}_{t}=\frac{\delta \mathscr{N}_{t}}{\mathscr{D}_{t}}-\frac{\mathscr{N}_{t} \delta \mathscr{D}_{t}}{\mathscr{D}_{t}^{2}}$, if inf $\mathscr{D}_{t}>0$.

Similarly for $\mathscr{N}_{i}$ and $\mathscr{B}_{i}$.

Finally, by lemma 19 , setting $z_{t}=\mathscr{B}_{t}, \vartheta=-\mathbb{1}_{[-1,0]}$ and $g_{t}=\eta+\sigma \mathfrak{r}_{t},(\mathscr{B}, \mathfrak{r}) \mapsto c$ is $S^{1}$ with derivative

$$
\delta c_{t}=\int_{t-1}^{t} \delta \mathscr{B}_{x} Z_{x, t} d x-\sigma \int_{t-1}^{t} \delta \mathfrak{r}_{x} \int_{t-1}^{x} \mathscr{B}_{s} Z_{s, t} d s d x
$$

Substituting for $\delta \mathscr{N}$ and $\delta \mathscr{D}$,

$$
\begin{aligned}
& \delta c_{t}=\int_{t-1}^{t} \frac{Z_{x, t}}{\mathscr{D}_{x}} \int\left[\left(\mathbb{1}_{x \leq z} \int_{z}^{\infty} X_{x, s} E_{s, s-x} d s\right) \delta \mathfrak{r}_{z}+X_{x, z} \delta E_{z, z-x}\right] d z d x+ \\
& \quad+\int_{t-1}^{t} \frac{Z_{x, t}}{\mathscr{D}_{x}}(1-\alpha) \int\left[\left(\mathbb{1}_{x \leq z} \int_{z}^{\infty} X_{x, s} y_{s} \varphi_{s-x} d s\right) \delta \mathfrak{r}_{z}+X_{x, z} \delta y_{z} \varphi_{z-x}\right] d z d x \\
& \quad+\int_{t-1}^{t} \frac{(\sigma-1) \mathscr{N}_{x} \int_{x}^{x+1} \delta \mathfrak{r}_{s} \int_{s}^{x+1} X_{x, z} Z_{x, z} d z d s}{\mathscr{D}_{x}^{2}} Z_{x, t} d x \\
& \quad+\int_{t-1}^{t} \delta \mathfrak{r}_{x} \int_{t-1}^{x}(-\sigma) \mathscr{B}_{s} Z_{s, t} d s d x
\end{aligned}
$$

$X_{x, t}$ and $Z_{x, t}$ are positive, bounded (as $\inf _{t} k_{t}>0$ implies $\mathfrak{r}$ is bounded) and, on $0 \leq t-x \leq 1$, are bounded away from 0 , thus also implying $\mathscr{D}_{x}$ is bounded away from zero, $E$ is locally integrable by assumption. It follows that $\mathrm{k}_{E}^{c}(t, x, u)$ is bounded. Also, since $\int_{z}^{\infty} X_{x, s} E_{s, s-x} d s=\int \mathbb{1}_{\max \{x, z\} \leq s \leq x+1} X_{x, s} E_{s, s-x} d s$ by definition of $X$, the left hand side of the equality is finite, continuous in $x, z$ and is zero for $z \geq x+1$. Hence it will remain unchanged when multiplied by $\mathbb{1}_{z \leq x+1}$. Then the second integral is equal to

$$
\int \mathbb{1}_{x \leq z \leq x+1}\left[\int \mathbb{1}_{\max \{x, z\} \leq s \leq x+1} X_{x, s} E_{s, s-x} d s \delta \mathfrak{r}_{z}+X_{x, z} \delta E_{z, z-x}\right] d z
$$

and is $<\infty$ by (ib). Hence, the outer integral in the first term (over $x \in[t-1, t]$ ) is finite too. Thus, Fubini applies to the first term (and to both of its summands).

Similarly, it applies to the second term since $\varphi$ is a measure with finite support and $y$ is bounded by feasibility [19, prop. 1.a]. Also, it applies to the rest of the terms since all the integrands there are locally integrable and are integrated over compact intervals. 
Now one can apply Fubini to each of the terms. Further, replacing $s$ with $u=s-x$, then $x$ with $v=t-x$, and $v$ with $x=t-v+u$ and $u$ with $s$,

$$
\iint \mathbb{1}_{x \leq z \leq s} \frac{Z_{x, t} X_{x, s}}{\mathscr{D}_{x}} E_{s, s-x} d s d x=\iint \mathbb{1}_{z \leq x \leq z+s} \frac{Z_{x-s, t} X_{x-s, x}}{\mathscr{D}_{x-s}} E_{x, s} d s d x
$$

The same transformation can be applied to the term containing $y_{s} \varphi_{s-x}$ (used in place of $E_{s, s-x}$ ) and to the terms containing $\delta E_{z, z-x}$ and $\delta y_{z} \varphi_{z-x}$ (without the indicator function).

Note also, that $\frac{Z_{x-s, t} X_{x-s, x}}{\mathscr{D}_{x-s}}=\mathrm{k}_{E}^{c}(t, x, x-s)$ by notation 5.1 .

Using points (ia), (ib), the notation for $\mathrm{k}_{E}^{c}$ and $\mathscr{B}$, and combining terms,

$$
\begin{aligned}
& \delta c_{t}=\iint \mathrm{k}_{E}^{c}(t, x, x-s) \delta E_{x, s} d s d x+ \\
& \quad+\alpha A(1-\alpha)\left(\iiint \mathbb{1}_{z \leq x \leq z+s} \mathrm{k}_{E}^{c}(t, x, x-s)\left[E_{x, s}+(1-\alpha) \varphi_{s} y_{x}\right] d s d x k_{z}^{\alpha-2} \delta k_{z} d z+\right. \\
& \quad+\iint \mathrm{k}_{E}^{c}(t, x, x-s) \varphi_{s} k_{x}^{\alpha-1} d s \delta k_{x} d x \\
& \quad+\iiint(\sigma-1) \mathbb{1}_{0 \leq t-x \leq 1} \mathbb{1}_{x \leq s \leq x+1} \mathbb{1}_{s \leq z \leq x+1} \mathscr{B}_{x} \mathrm{k}_{E}^{c}(t, z, x) Z_{x, z} d x d z \delta k_{s}^{\alpha-2} \delta k_{s} d s \\
& \left.\quad-\iint \sigma \mathbb{1}_{0 \leq t-x \leq 1} \mathbb{1}_{t-1 \leq s \leq x} \mathscr{B}_{s} Z_{s, t} d s k_{x}^{\alpha-2} \delta k_{x} d x\right)
\end{aligned}
$$

Using

$$
\begin{aligned}
& \text { (ב) } \quad \mathrm{k}_{1}^{c}(t, z) \stackrel{\text { def }}{=} \iint \mathbb{1}_{z \leq x \leq z+s} \mathrm{k}_{E}^{c}(t, x, x-s)\left[E_{x, s}+(1-\alpha) \varphi_{s} y_{x}\right] d s d x \\
& \mathrm{k}_{2}^{c}(t, x) \stackrel{\text { def }}{=} k_{x} \int \mathrm{k}_{E}^{c}(t, x, x-s) \varphi_{s} d s \\
& \mathrm{k}_{3}^{c}(t, s) \stackrel{\text { def }}{=} \iint(\sigma-1) \mathbb{1}_{0 \leq t-x \leq 1} \mathbb{1}_{x \leq s \leq x+1} \mathbb{1}_{s \leq z \leq x+1} \mathscr{B}_{x} \mathrm{k}_{E}^{c}(t, z, x) Z_{x, z} d x d z \\
& \mathrm{k}_{4}^{c}(t, x) \stackrel{\text { def }}{=}-\int \sigma \mathbb{1}_{0 \leq t-x \leq 1} \mathbb{1}_{t-1 \leq s \leq x} \mathscr{B}_{s} Z_{s, t} d s \\
& \mathrm{k}_{k}^{c}(t, x) \stackrel{\text { def }}{=} \alpha A(1-\alpha) k_{x}^{\alpha-2} \sum_{j=1}^{4} \mathrm{k}_{j}^{c}(t, x)=\left(R-\mathfrak{r}_{x}\right) \frac{1-\alpha}{k_{x}} \sum_{j=1}^{4} \mathrm{k}_{j}^{c}(t, x)
\end{aligned}
$$

get, finally,

$$
\delta c_{t}=\iint \mathrm{k}_{E}^{c}(t, x, x-s) \delta E_{x, s} d s d x+\int \mathrm{k}_{k}^{c}(t, v) \delta k_{v} d v
$$

$\mathrm{k}_{1}^{c}$ is bounded, since $\mathrm{k}_{E}^{c}$ and $y$ are, $E \in L_{\infty, 1}$ and $\varphi$ is a finite measure. Then also $\mathrm{k}_{2}^{c}$ is bounded because $k$ is so (by feasibility, [19, prop. 1.a]). In addition, it follows that $\mathscr{N}_{1, x}=\int X_{x, x+s} E_{x, s} d s, \mathscr{N}_{2, x}=\int X_{x, x+s} \varphi_{s} y_{x+s} d s$ and $Z$ are also bounded. Since $\mathscr{D}_{x}$ is bounded and bounded away from zero ( $\inf _{t} k_{t}>0, \mathfrak{r}$ bounded), it then follows that $\mathscr{B}$ is so. Hence $\mathrm{k}_{3}^{c}$ and $\mathrm{k}_{4}^{c}$ are bounded too, which then implies boundedness of $\mathrm{k}_{k}^{c}$ (again, since $\inf _{t} k_{t}>0$ ).

Finally, we show that $\mathrm{k}_{k}^{c}$ is continuous, which follows from continuity of $\mathrm{k}_{j}^{c}$ for $j=1, \ldots, 4$ (cf. display $\beth$ ) shown below. All but one of them depend on $\mathrm{k}_{E}^{c}(z, x, u)$, which is non-zero only if $z-u \in[0,1]$ and $x-u \in[0,1]$. It is discontinuous only at $z=u$ and $x=u$.

$\mathrm{k}_{1}^{c}(z, x)=\iint \mathbb{1}_{x \leq u \leq x+s} \mathrm{k}_{E}^{c}(z, u, u-s)\left[E_{u, s}+(1-\alpha) \varphi_{s} y_{u}\right] d s d u$ : the integrand, without changing its value, can be multiplied by $\mathbb{1}_{0 \leq z-u+s \leq 1}$ and by $\mathbb{1}_{0 \leq s \leq 1}$ by definition of $\mathrm{k}_{E}^{c}$. It implies that $\mathrm{k}_{1}^{c}(z, x)$ is zero for $z-x \notin[-1,1]$. The term $\left[E_{u, s}+(1-\alpha) \varphi_{s} y_{u}\right]$ is in $L_{\infty, 1}$, and is integrated over a bounded region, $u \in$ $[\max \{x, z+s-1\}, \min \{x+s, z+s\}], s \in[0,1]$ for any fixed $x, z \cdot \mathrm{k}_{E}^{c}(z, u, u-s)$ is continuous in (the interior of) that region and is bounded by point (ic). Equivalently, since Fubini is applicable, the first integral could be taken over $s \in[\max \{u-$ $x, u-z\}, \min \{1,1+u-z\}]$, while the second one over $u \geq x$. Hence the first integral is a continuous function of the boundary, $\max \{u-x, u-z\} \geq 0$ and therefore is a continuous function of $u, z, x$. Similarly, the outer integral is the continuous function of its boundary, $x$, and of $z$ (as the bounded integrand is so).

$\mathrm{k}_{2}^{c}(z, x)=k_{x} \int \mathrm{k}_{E}^{c}(z, x, x-s) \varphi_{s} d s$ : again the integrand, without changing its value, can be multiplied by $\mathbb{1}_{0 \leq z-x+s \leq 1}$ and by $\mathbb{1}_{0 \leq s \leq 1}$ by definition of $\mathrm{k}_{E}^{c}$. It 
implies that $\mathrm{k}_{2}^{c}(z, x)$ is zero for $z-x \notin[-1,1] . \varphi$ is a finite measure integrated over its support, $[0,1]$, and $\mathrm{k}_{E}^{c}$ is bounded (point (ic)) and is continuous inside the bounds of integration: $s \in[\max \{0, x-z\}, \min \{1,1+x-z\}]$. Hence, since $k_{x} \in C_{\infty}$, $\mathrm{k}_{2}^{c}(z, x)$ is continuous in both arguments.

$\mathrm{k}_{3}^{c}(z, x)=\iint(\sigma-1) \mathbb{1}_{0 \leq z-u \leq 1} \mathbb{1}_{u \leq x \leq u+1} \mathbb{1}_{x \leq s \leq u+1} \mathscr{B}_{u} \mathrm{k}_{E}^{c}(z, s, u) Z_{u, s} d u d s$ : the integrand is bounded, as each term $\left(\mathscr{B}, \mathrm{k}_{E}^{c}, Z\right)$ is so by above (cf. the proof of (ic)). $\mathscr{B}, Z$ are continuous and $\mathrm{k}_{E}^{c}(z, s, u)$ is so in the interior of the region $z-u \in[0,1]$ and $s-u \in[0,1]$, beyond which it is zero, and hence is continuous within the bounds of integration. This along with the boundedness of the integrand and the bounds of the inner integral: $u \in[\max \{z-1, x-1, s-1\}, \min \{z, x, s\}]$ implies that the inner integral is continuous in $x, z, s$, while the outer integral is over $s \geq x$ and hence is continuous in $x, z$. It also follows that $\mathrm{k}_{3}^{c}(z, x)=0$ for $x-z \notin[-1,1]$.

$\mathrm{k}_{4}^{c}(z, x)=-\sigma \int \mathbb{1}_{0 \leq z-x \leq 1} \mathbb{1}_{z-1 \leq s \leq x} \mathscr{B}_{s} Z_{s, z} d s$ : similarly, the integrand is bounded and continuous and the integral is a continuous function of the boundaries and hence of $x, z$.

It follows $\mathrm{k}_{k}^{c}(z ; x)$ is continuous and is zero for $z-x \notin[-1,1]$.

id: by lemma 13 .

ie: by $[20$, cor. 8$]$.

if: by lemma 20 .

ii: By [20, prop. 5].

v: One can consider partial derivatives for each of the maps (ia)-(if) (e.g., $\delta k \mapsto\left[t \curvearrowright \int \mathrm{k}_{k}^{c}(t, z) \delta k_{z} d z\right]$ for (ic)) separately, because by definition each satisfies the Lipschitz condition implied by the $S^{1}$ property to the appropriate $\mathbb{L}$ space, and the sums are valid in those spaces. To get to the simpler norm employed in the proposition, use then prop. 6 .

iii and iv: By points (ic)-(if),

$$
\begin{aligned}
\delta \tilde{k}_{t} & =\int_{-\infty}^{t} e^{-R(t-z)}\left(\delta y_{z}+\int \delta E_{z, s} d s-\delta c_{z}\right) d z= \\
& =\int_{-\infty}^{t} e^{-R(t-z)}\left(\int \delta E_{z, s} d s-\iint \mathrm{k}_{E}^{c}(z ; x, x-s) \delta E_{x, s} d x d s\right) d z+ \\
& +\int_{-\infty}^{t} e^{-R(t-z)}\left(\alpha A k_{z}^{\alpha-1} \delta k_{z}-\int \mathrm{k}_{k}^{c}(z, x) \delta k_{x} d x\right) d z
\end{aligned}
$$

Since $\xi_{R}(x)$ is a positive finite measure, its convolution with a bounded function is finite, and since its convolution with $\delta E \in L_{\infty, 1}$ can be majorised by a constant times $\sum_{n=1}^{+\infty} e^{-R n}<\infty$, Fubini is applicable to all the terms. Then, denoting by $\epsilon_{x}$ the unit mass at $x$,

$$
\begin{aligned}
\delta \tilde{k}_{t} & =\iiint_{-\infty}^{t} e^{-R(t-z)}\left(\epsilon_{x}(z)-\mathrm{k}_{E}^{c}(z ; x, x-s)\right) d z \delta E_{x, s} d x d s+ \\
& +\iint_{-\infty}^{t} e^{-R(t-z)}\left(\alpha A k_{z}^{\alpha-1} \epsilon_{x}(z)-\mathrm{k}_{k}^{c}(z ; x)\right) d z \delta k_{x} d x
\end{aligned}
$$

So, with

$$
\begin{aligned}
& \xi_{R}(x) \stackrel{\text { def }}{=} \mathbb{1}_{x \geq 0} e^{-R x}, \\
& \mathrm{k}_{E}^{k d}(t, x) \stackrel{\text { def }}{=} \int \xi_{R}(t-z) \epsilon_{x}(z) d z, \quad \mathrm{k}_{k}^{k d}(t ; x) \stackrel{\text { def }}{=} \int \xi_{R}(t-z) \alpha A k_{z}^{\alpha-1} \epsilon_{x}(z) d z \\
& \mathrm{k}_{E}^{k c}(t, x, u) \stackrel{\text { def }}{=} \int \xi_{R}(t-z) \mathrm{k}_{E}^{c}(z ; x, u) d z \quad \mathrm{k}_{k}^{k c}(t ; x) \stackrel{\text { def }}{=} \int \xi_{R}(t-z) \mathrm{k}_{k}^{c}(z ; x) d z \\
& \mathrm{k}_{E}^{k}(t ; x, u) \stackrel{\text { def }}{=} \mathrm{k}_{k}^{k d}(t ; x)-\mathrm{k}_{k}^{k c}(t ; x, u), \quad \mathrm{k}_{k}^{k}(t ; x) \stackrel{\text { def }}{=} \mathrm{k}_{k}^{k d}(t ; x)-\mathrm{k}_{k}^{k c}(t ; x)
\end{aligned}
$$


we get

$$
\delta \tilde{k}_{t}=\iint \mathrm{k}_{E}^{k}(t ; x, x-s) \delta E_{x, s} d x d s+\int \mathrm{k}_{k}^{k}(t ; x) \delta k_{x} d x
$$

Hence the formulae in (iii) and (iv).

vi: $\mathrm{k}_{k}^{k c}$ is Lipschitz in $t$ to $L_{\infty}$ by cor. 10 . It is continuous, since by point (ic), $\mathrm{k}_{k}^{c}(z ; x)$ is continuous and is zero for $z-x \notin[-1,1]$. The latter along with boundedness of $\mathrm{k}_{k}^{c}(z ; x)$ (point (ic)), say, by some $Q$, implies $\mathrm{k}_{k}^{k c}(t ; x) \leq Q \int_{x-1}^{x+1} \xi_{R}(t-z) d z$. $\xi$ being a non-decreasing function, the bound for $\mathrm{k}_{k}^{k c}$ is as stated.

Since $\xi$ is bounded, it then follows that $\mathrm{k}_{k}^{k c}$ is bounded and since $\frac{y}{k}$ is bounded, $\mathrm{k}_{k}^{k d}$ and hence $\mathrm{k}_{k}^{k}$ are bounded too.

vii: Similarly, since $\mathrm{k}_{E}^{k c}(t, x, x-s)=\int \xi_{R}(t-z) \mathrm{k}_{E}^{c}(z ; x, x-s) d z$ and $\mathrm{k}_{E}^{c}(z ; x, x-s)$ is bounded (by some $D$ ) and is zero for $z-x \notin[0,1]$ and $s \notin[0,1], \mathrm{k}_{E}^{k c}(t, x, x-s) \leq$ $\mathbb{1}_{s \in[0,1]} D \int_{x}^{x+1} \xi_{R}(t-z) d z$ and by monotonicity of $\xi$ the bound can be taken as claimed. Also, similarly to the previous point, Lipschitz property in $t$ follows from corollary 10 .

Now let us show the Lipschitz property of $\mathrm{k}_{E}^{k c}(t, x, u)$ with respect to $u$. Since it is non-zero only for $t-u \in[0,1]$, take $h \in[0,1]$ then, $k_{E}^{c}$ being bounded (ic) (by $D)$, and $\xi_{R}(t-z) \leq 1,\left|\mathrm{k}_{E}^{k c}(t, x, u+h)-\mathrm{k}_{E}^{k c}(t, x, u)\right| \leq 2 D h+\int_{u+h}^{\min \{t, u+1\}} \mid \mathrm{k}_{E}^{c}(z ; x, u+$ $h)-\mathrm{k}_{E}^{c}(z ; x, u) \mid d z$, for $(s=) x-u \in[0,1]$. The integral is maximal for $t \geq u+1$ : we have to bound $\int_{u}^{u+1}\left|\mathrm{k}_{E}^{c}(z ; x, u+h)-\mathrm{k}_{E}^{c}(z ; x, u)\right| d z$. Bound the integral by a sup $z$, then for each $x$ the absolute value of the increment between $u$ and $u+h$ is bounded by $h$ times the sup of the derivative between $u$ and $u+h\left(\mathrm{k}_{E}^{c}(z ; x, u+h)\right.$ being obviously differentiable w.r.t. $h$ ), and the derivative is bounded for $x-u \in[0,1]$ by e.g. $\leq 4 A e^{4 A}\left(\frac{1}{\inf _{x} \mathscr{D}_{x}}-\frac{1}{\sup _{x} \mathscr{D}_{x}^{2}}\right)$, where $A=\mid(1-\sigma)$ sup $\mathfrak{r}_{s}-\eta \mid$. The proof of the Lipschitz property of $\mathrm{k}_{E}^{k c}(t, x, u)$ with respect to $x$ is similar.

viii: By (vii), $\mathrm{k}_{E}^{k}(\mu ; \cdot, \cdot)$ is well-defined, and Borel measurable. Also, both terms of $\mathrm{k}_{E}^{k}(x ; t, s)$ are bounded by $\mathbb{1}_{s \in[0,1]} \xi_{R}(x-t+1)$ times a constant; so $\left|\mathrm{k}_{E}^{k}(\mu ; t, s)\right| \leq$ $\int \xi_{R}(x-t+1)|\mu|(d x)=\left(\bar{\xi}_{R} \star|\mu|\right)(t-1)$, and thus $\left\|\mathrm{k}_{E}^{k}(\mu ; \cdot, \cdot)\right\|_{1, \infty} \leq\left\|\bar{\xi}_{R} \star|\mu|\right\|_{1, \infty} \leq$ $\|\mu\|\left\|\xi_{R}\right\|_{1, \infty}$ by $[20$, thm. 2.ii]. Hence the boundedness.

For the continuity, since bounded sets of $M$ are metrisable, consider $\mu_{n} \rightarrow \mu$. For the $\mathrm{k}_{E}^{k}\left(\mu_{n} ; \cdot, \cdot\right)$, the boundedness part in the definition of tightness [20, def. 2] is satisfied, by the above. To verify the second part of the definition, extract a converging subsequence from $\left|\mu_{n}\right|$; the continuity statement of [20, thm. 2.iii] yields, in the above $\left|\mathrm{k}_{E}^{k}\left(\mu_{n} ; t, s\right)\right| \leq\left(\bar{\xi}_{R} \star\left|\mu_{n}\right|\right)(t-1)$, that the right hand member is $\|\cdot\|_{1, \infty}$ convergent, hence tight $\left([20\right.$, prop. 1. iii] $)$; thus so are the $\mathrm{k}_{E}^{k}\left(\mu_{n} ; \cdot, \cdot\right)$. Finally, as to convergence a.e., give $(x, s)$, the only discontinuity in $x$ of $\mathrm{k}_{E}^{k}(t ; x, s)$ stems from the first term involving $\mathrm{k}_{E}^{k d}(t ; x, s)$, and is for $t=x$. Hence, for all $(x, s)$ s.t. $x$ is not an atom of $\mu, \mathrm{k}_{E}^{k}\left(\mu_{n} ; x, s\right) \rightarrow \mathrm{k}_{E}^{k}(\mu ; x, s)$. Hence the continuity, by [20, prop. 1.iv].

xii: The second part follows from (vii). By the above argument, it implies the convergence is uniform. By [20, prop. 1.iv], this together with the tightness implied by Mackey convergence implies norm convergence.

x: viii implies $\left\|\varrho_{E}^{k}(\mu(\omega) ; \cdot, \cdot)\right\|_{1, \infty} \leq K\|\mu(\omega)\| ;$ so $\int\left|\varrho_{E}^{k}(\mu(\omega) ; t, t-s s)\right|\left|f_{t, s}\right| d s d t$ is integrable in $\omega$ by [20, thm. 1.xiii]. Use then Fubini.

For the last item, use $\mu$ there as $\nu$, and $x \mapsto \epsilon_{x}$ on $\mathbb{R}$ as bounded measurable map.

ix: If $\mu_{n} \geq 0$ and $\mu$ is non-atomic, consider $\left(\bar{\xi}_{R} \star \mu_{n}\right)(x)=e^{R t} \int_{x \geq t} e^{-R x} \mu_{n}(d x)$ : the integral is monotone, and converges pointwise to its continuous limit, so by Dini's theorem the convergence is uniform on compact sets: $\bar{\xi}_{R} \star \mu_{n} \rightarrow \bar{\xi}_{R} \star \mu$ uniformly on compact sets. Thus the conclusion, as above. The continuity of $\varrho_{E}^{k}(\mu ; \cdot, \cdot)$ for $\mu$ non-atomic is classical (dominated convergence). 


\section{Generic invertibility of $\frac{\partial F}{\partial k}$ AT A BGE}

Recall, to establish regularity and stability we have to verify invertibility of the derivative of the equilibrium map, $\partial F(k, 0) / \partial k$ at a BGE $k$. So, first, we characterise BGE without transfers, or "status-quo" equilibria.

6.1. BGE and the equilibrium graph. The characterisation is based on cor. 13 and remark 24 in [19], but it can also be viewed (and proven as) a corollary to proposition 1.

Notation 6.1. Let $\Phi(z) \stackrel{\text { def }}{=} \frac{e^{z}-1}{z}, \digamma(\mathfrak{r}) \stackrel{\text { def }}{=} \frac{\Phi(-\mathfrak{r} \sigma-\eta)}{\Phi(\mathfrak{r}(1-\sigma)-\eta)}$.

Corollary 3. The set of BGE is the set of constant solutions $\mathfrak{r}$ of the system

$$
\mathfrak{r}\left(\frac{1-\digamma(\mathfrak{r}) \int e^{\mathfrak{r} s} \varphi(d s)}{\mathfrak{r}}(R-\mathfrak{r})-\frac{\alpha}{1-\alpha}\right)=0
$$

with the rest of the (constant) quantities determined by:
(i) $k=\left[\frac{R-\mathfrak{r}}{A \alpha}\right]^{\frac{1}{\alpha-1}}$
(ii) $y=A k^{\alpha}$
(iii) $i=R k$
(iv) $\mathscr{N}=(1-\alpha) y \int_{0}^{1} e^{\mathfrak{r} s} \varphi_{s} d s$
(v) $c=\digamma(\mathfrak{r}) \mathscr{N}$
(vi) $\mathscr{D}=\Phi(\mathfrak{r}(1-\sigma)-\eta), \mathscr{B}=\frac{\mathscr{N}}{\mathscr{D}}$

Remark 4. Recall, the prices: $p_{t}=p_{0} e^{(\mathfrak{r}-\gamma-\nu) t}, w_{t}=(1-\alpha) e^{\gamma t} p_{t}, r_{t}=p_{t}(R-\mathfrak{r})$ are fully determined by $\mathfrak{r}$ and are omitted from the list of the equilibrium variables.

Remark 5. Number BGE in this economy is finite by [19, thm. 3] since the production function $f(k)=A k^{\alpha}$ satisfies assumption 5 there and is analytic.

The invertibility will hold generically in the following sense: for any choice of parameters out of a generic set [cf. def.3], at any BGE the derivative is invertible. So, to take care of multiple equilibria, we have to consider parameters-equilibrium pairs, or an equilibrium graph:

Definition 8. Let $\mathfrak{G}$ be the cross product of the parameter space, $\wp$, and $P=$ $C_{\infty}^{4} \times L_{\infty, 1}$, containing the equilibrium variables: $(k, y, c, \mathfrak{r}, i)$. The equilibrium graph (restricting attention to BGE) is the subset $G$ of $\mathfrak{G}$ composed of all points satisfying cor. 3 .

To illustrate, we borrow (with slight modifications) from [19] the projection of such graphs into the space of a single parameter $\alpha$ and an equilibrium variable, $1-\mathfrak{r} / R$. The line, of course, indicates combinations of $\alpha$ and $\mathfrak{r}$ that constitute a BGE (while the rest of the parameters are fixed).

Figures $1-4$ show the BGE of economies with $\varphi(s)=\frac{1}{b-a} \mathbb{1}_{[a, b]}(s)$ and reasonable parameters (time unit being 1 lifetime).

Heuristically, what are the "bad points" on the equilibrium graph, i.e., the points where the invertibility fails? (We also call the set of such points exceptional further.) Since $\partial F / \partial k$ equals $\partial \Upsilon / \partial k$ minus the identity, $\partial F / \partial k$ is not invertible if and only if unity is in the spectrum ${ }^{18}$ of $\partial \Upsilon / \partial k$. But the latter is true only if the range of the Fourier transform of the (convolution) kernel of $\partial \Upsilon / \partial k$ contains 1 , so, if it does, this is a "bad point" of the graph. This heuristic, of course, is based on the extension of the Wiener theorem, prop. 2. So, the next step is to inspect $\partial \Upsilon / \partial k$ at a BGE, verify it is a convolution and calculate the Fourier transform of the kernel.

\footnotetext{
${ }^{18} \mathrm{~A}$ complex number $z \in \mathbb{C}$ is in the spectrum of an element $A$ of a Banach algebra if $A-z I$ is not invertible, where $I$ is the unit element of the algebra (cf. e.g., [16, IV, §2, p. 68]).
} 


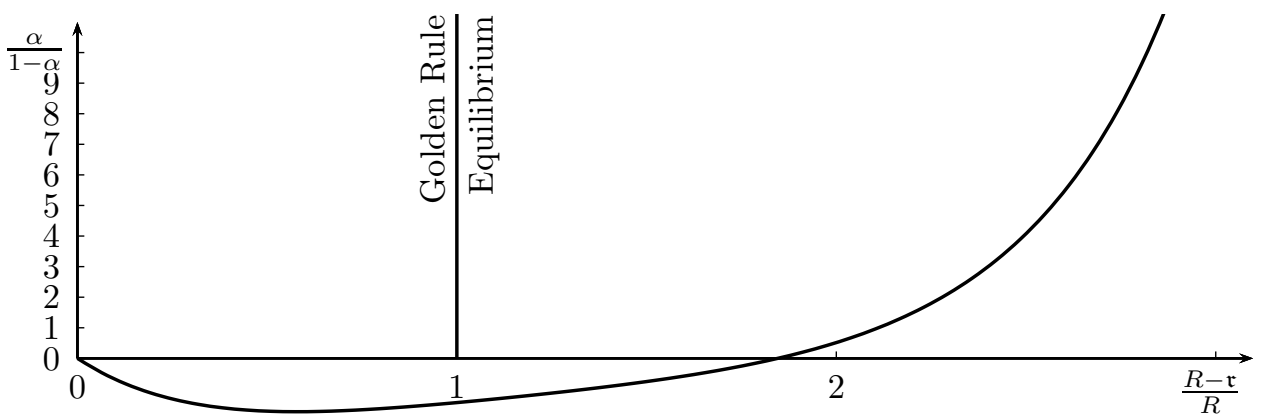

Figure 1. $R=11, \sigma=.5, \eta=2, a=.2, b=.75$. Two equilibria $\forall \alpha$.

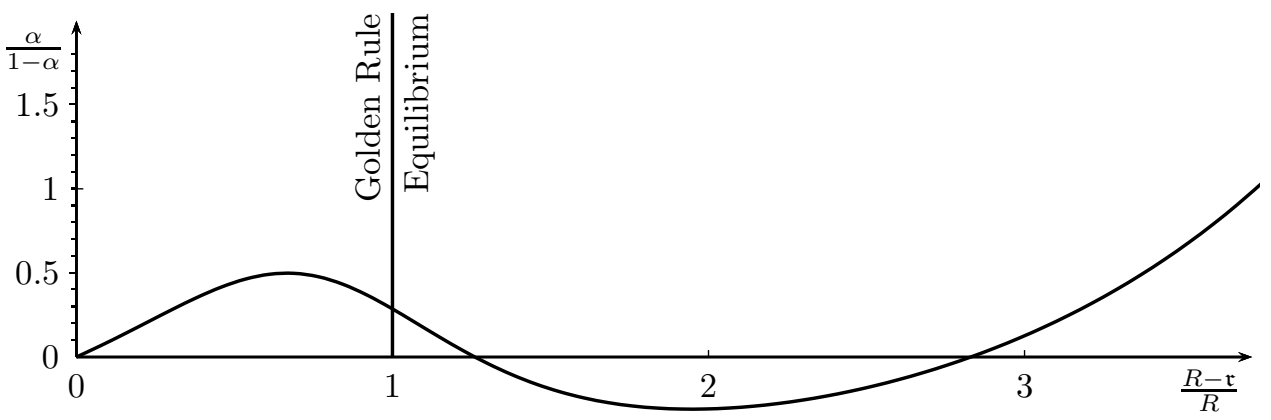

FiguRE 2. $R=11, \sigma=.25, \eta=2, a=.135, b=.5$. Two to four equilibria.

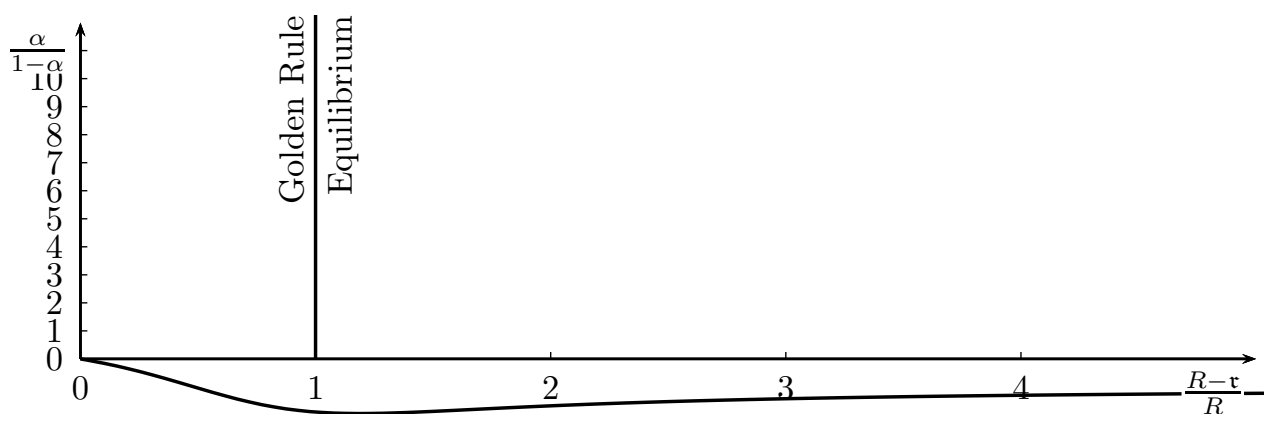

Figure 3. $R=10, \sigma=.25, \eta=2.5, a=.25, b=.75 .1$ equilibrium $\forall \alpha$.

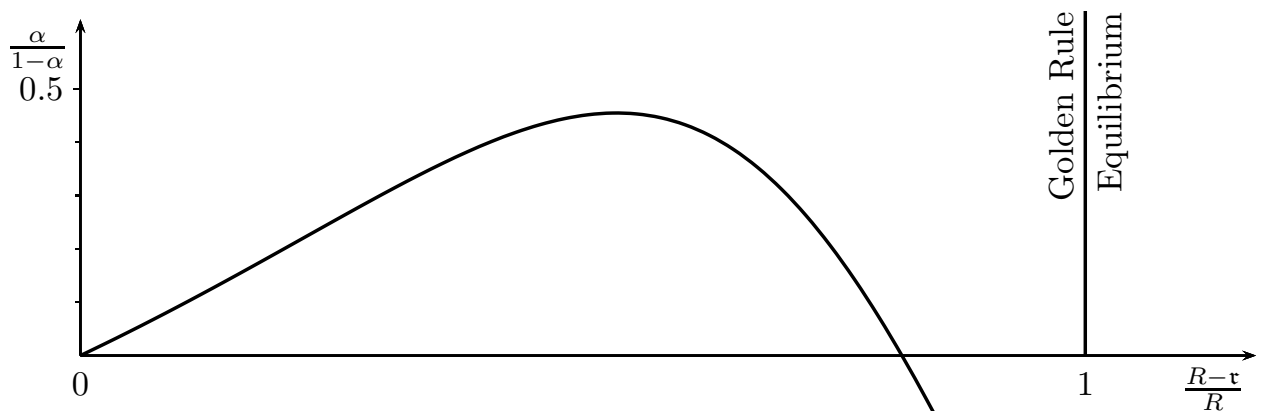

Figure $4 . R=15, \sigma=.24, \eta=1.9, a=.24, b=.55 .1$ or 3 equilibria. 


\subsection{Derivative of the equilibrium map $\frac{\partial \Upsilon}{\partial k}$ at a BGE.}

Notation 6.2. $\psi_{t}=\mathbb{1}_{0 \leq t \leq 1} \int_{t}^{1} e^{\mathfrak{r} s} \varphi_{s} d s, \quad \chi_{t}^{x}=\mathbb{1}_{0 \leq t \leq 1}\left(1-\frac{t \Phi(x t)}{\Phi(x)}\right)$

$$
\begin{aligned}
\Phi(z) & =\frac{e^{z}-1}{z} & \Psi(x, y) & =\frac{\Phi(-x+\mathrm{i} y)}{\Phi(-x)} & v(z) & =\int_{0}^{1} e^{z t} \varphi(d t) \\
\varkappa & =\mathfrak{r} \sigma+\eta & B & =\frac{\alpha}{(1-\alpha)(R-\mathfrak{r})} & C & =\frac{(1-\alpha) \Phi(-\varkappa)}{B \Phi(\mathfrak{r}-\varkappa)}
\end{aligned}
$$

Lemma 2. (i) At a $B G E\left(\frac{\partial y}{\partial k}\right)(\delta k)=(R-\mathfrak{r}) \delta k, \quad\left(\frac{\partial \mathfrak{r}}{\partial y}\right)(\delta y)=(1-\alpha) \frac{\delta y}{k}$.

(ii) The kernel $\mathrm{k}_{k}^{c}(t, z)$ from lemma 1.ic at a BGE is a convolution kernel, ${ }^{19}$ $\mathbb{R}_{k}^{c}(t-z) \stackrel{\text { def }}{=} \mathrm{k}_{k}^{c}(t, z)$.

(iii) The derivative $T=\frac{\partial \Upsilon}{\partial k}\left(k^{*}, 0\right)$ at a $B G E$ is a convolution operator, with kernel $\mathbb{k}_{k}^{k} \in L_{1}^{\lambda} \forall \lambda<R$ having as FT $\widehat{\mathbb{k}}_{k}^{k}=\frac{R-\mathfrak{r}}{R-\mathrm{i} \omega}(1-\widehat{H})$, where $H: w \mapsto$ $\frac{1}{R-\mathrm{r}} \mathbb{k}_{k}^{c}(w)$, so

$$
\begin{aligned}
& \widehat{H}(\omega)=\Phi(-\varkappa+\mathrm{i} \omega) \widehat{Q}(-\omega)-C \sigma v(\mathfrak{r}) \widehat{\chi}^{-\varkappa}(\omega) \\
& \widehat{Q}(\omega)=\frac{C}{\Phi(-\varkappa)}\left[\widehat{\psi}(\omega)+B v(\mathfrak{r}+\mathrm{i} \omega)-(1-\sigma) v(\mathfrak{r}) \widehat{\chi}^{\mathfrak{r}-\varkappa}(\omega)\right] \\
& \widehat{\psi}(\omega)=\frac{1}{\mathrm{i} \omega}(v(\mathfrak{r}+\mathrm{i} \omega)-v(\mathfrak{r})), \quad \widehat{\chi}^{x}(\omega)=\frac{1}{\mathrm{i} \omega}(\Psi(-x, \omega)-1)
\end{aligned}
$$

or, equivalently,

(5) $\widehat{H}(\omega)=\frac{C}{\mathrm{i} \omega}(\Psi(\varkappa, \omega)[(B \mathrm{i} \omega-1) v(\mathfrak{r}-\mathrm{i} \omega)+(1-\sigma) v(\mathfrak{r}) \Psi(\varkappa-\mathfrak{r},-\omega)]+v(\mathfrak{r}) \sigma)$

(iv) $H \in L_{p}([-1,1]), p<\infty$, is norm-continuous on the BGE graph $G$.

Proof. Point i: by lemma 1 and cor. 3 .

(ii): To evaluate $\mathrm{k}_{k}^{c}$ at a BGE we use display $\beth$ in the proof of lemma 1. All of the terms depend on $\mathrm{k}_{E}^{c}$. First, note that with $\phi_{\mathfrak{r}}(s) d s \stackrel{\text { def }}{=} \mathbb{1}_{0 \leq s \leq 1} e^{s \mathfrak{r}} \varphi_{s} d s$, and $h_{-\varkappa}(s) \stackrel{\text { def }}{=} \mathbb{1}_{0 \leq s \leq 1} e^{-\varkappa s}$,

$$
\Phi(\mathfrak{r}-\varkappa) \int \mathrm{k}_{E}^{c}(t, x, x-s) \varphi_{s} d s=\int h_{-\varkappa}(t-x+s) \phi_{\mathfrak{r}}(s) d s=\left(h_{-\varkappa} \star \bar{\phi}_{\mathfrak{r}}\right)(t-x)
$$

So, $\mathrm{k}_{2}^{c}(t, x)$ at a BGE depends only on the difference, $t-x$, hence let $\mathbb{k}_{2}^{c}(t-x) \stackrel{\text { def }}{=}$ $\mathrm{k}_{2}^{c}(t, x)$, then $\mathbb{k}_{2}^{c}(w)=\frac{k}{\Phi(\mathfrak{r}-\varkappa)}\left(h_{-\varkappa} \star \bar{\phi}_{\mathfrak{r}}\right)(w)$. Next,

$$
\begin{aligned}
\mathrm{k}_{1}^{c}(t, z) & =\frac{(1-\alpha) y}{\Phi(\mathfrak{r}-\varkappa)} \iint \mathbb{1}_{z \leq x \leq z+s} h_{-\varkappa}(t-x+s) \phi_{\mathfrak{r}}(s) d s d x= \\
& =\frac{(1-\alpha) y}{\Phi(\mathfrak{r}-\varkappa)} \iint \mathbb{1}_{0 \leq t-z-u \leq s} h_{-\varkappa}(u+s) \phi_{\mathfrak{r}}(s) d s d u
\end{aligned}
$$

which, with $w=t-z$, can be written as

$$
\begin{aligned}
\mathbb{k}_{1}^{c}(w) & \stackrel{\text { def }}{=} \frac{(1-\alpha) y}{\Phi(\mathfrak{r}-\varkappa)} \iint \mathbb{1}_{0 \leq w-u \leq s} h_{-\varkappa}(u+s) \phi_{\mathfrak{r}}(s) d s d u, t=u+s \\
& =\frac{(1-\alpha) y}{\Phi(\mathfrak{r}-\varkappa)} \int h_{-\varkappa}(t) \mathbb{1}_{0 \leq t-w \leq 1} \int \mathbb{1}_{t-w \leq s} \phi_{\mathfrak{r}}(s) d s d t=\frac{(1-\alpha) y}{\Phi(\mathfrak{r}-\varkappa)}\left(h_{-\varkappa} \star \bar{\psi}\right)(w)
\end{aligned}
$$

The third term, $\mathrm{k}_{3}^{c}(t, s)$ evaluated at a BGE is $\frac{(1-\alpha)(\sigma-1) y v(\mathfrak{r})}{\Phi^{2}(\mathfrak{r}-\varkappa)} \iint \mathbb{1}_{0 \leq t-x \leq 1} e^{-\varkappa(t-x)} \mathbb{1}_{x \leq s \leq x+1} \mathbb{1}_{s \leq z \leq x+1} \mathbb{1}_{0 \leq z-x \leq 1} e^{\mathfrak{r}(z-x)} e^{-\varkappa(z-x)} d x d z$

\footnotetext{
${ }^{19}$ When a partial derivative of a map $v$ with respect to a variable $z$ is a convolution, denote the corresponding kernel by $\mathbb{k}_{z}^{v}$ so the derivative is $\left(\frac{\partial v}{\partial z}(\delta z)\right)_{x}=\int \mathbb{k}_{z}^{v}(x-t) \delta z_{t} d t$.
} 
After changing variables: $u=t-x$ and then $x=z-t$, it equals

$$
\frac{(1-\alpha)(\sigma-1) y v(\mathfrak{r})}{\Phi(\mathfrak{r}-\varkappa)} \int \mathbb{1}_{0 \leq u \leq 1} e^{-\varkappa u} \mathbb{1}_{-u \leq s-t \leq 1-u} \frac{e^{(\mathfrak{r}-\varkappa)}-e^{(\mathfrak{r}-\varkappa)(s-t+u)}}{e^{\mathfrak{r}-\varkappa}-1} d u
$$

Now it can be written as a function of $w=t-s$ :

$\mathbb{k}_{3}^{c}(w)=\frac{(1-\alpha)(\sigma-1) y v(\mathfrak{r})}{\Phi(\mathfrak{r}-\varkappa)} \int \mathbb{1}_{0 \leq u \leq 1} e^{-\varkappa u} \chi_{u-w}^{\mathfrak{r}-\varkappa} d u=\frac{(1-\alpha)(\sigma-1) y v(\mathfrak{r})}{\Phi(\mathfrak{r}-\varkappa)}\left(h_{-\varkappa} \star \bar{\chi}^{\mathfrak{r}-\varkappa}\right)(w)$

In a BGE, by cor. 3.i-ii, $\frac{y}{k}=\frac{R-\mathfrak{r}}{\alpha}$, so with $Q(w) \stackrel{\text { def }}{=} \frac{C}{\Phi(-\varkappa)}\left(\psi(w)+\frac{\alpha \phi_{\mathfrak{r}}(w)}{(1-\alpha)(R-\mathfrak{r})}+\right.$ $\left.(\sigma-1) v(\mathfrak{r}) \chi_{w}^{\mathfrak{r}-\varkappa}\right)$

$$
\mathbb{k}_{1}^{c}(w)+\mathbb{k}_{2}^{c}(w)+\mathbb{k}_{3}^{c}(w)=\frac{k}{1-\alpha} h_{-\varkappa} \star \bar{Q}(w)
$$

Next,

$$
\begin{aligned}
\mathrm{k}_{4}^{c}(t, x) & =-\frac{(1-\alpha) y \sigma v(\mathfrak{r})}{\Phi(\mathfrak{r}-\varkappa)} \int \mathbb{1}_{0 \leq t-x \leq 1} \mathbb{1}_{0 \leq t-s \leq 1} \mathbb{1}_{t-1 \leq s \leq x} e^{-\varkappa(t-s)} d s \\
& =-\frac{(1-\alpha) y \sigma v(\mathfrak{r})}{-\varkappa \Phi(\mathfrak{r}-\varkappa)} \mathbb{1}_{0 \leq t-x \leq 1}\left(e^{-\varkappa}-e^{-\varkappa(t-x)}\right) \\
& =-\frac{(1-\alpha) y \sigma v(\mathfrak{r}) \Phi(-\varkappa)}{\Phi(\mathfrak{r}-\varkappa)} \mathbb{1}_{0 \leq t-x \leq 1} \frac{\left(e^{-\varkappa}-e^{-\varkappa(t-x)}\right)}{e^{-\varkappa}-1}= \\
\mathbb{k}_{4}^{c}(t-x) & \stackrel{\text { def }}{=}-\frac{(1-\alpha) y \sigma v(\mathfrak{r}) \Phi(-\varkappa)}{\Phi(\mathfrak{r}-\varkappa)} \chi_{t-x}^{-\varkappa}
\end{aligned}
$$

Using, again, cor. 3.i-ii,

$$
\mathbb{k}_{4}^{c}(w)=-\frac{(1-\alpha) k(R-\mathfrak{r}) \sigma v(\mathfrak{r}) \Phi(-\varkappa)}{\alpha \Phi(\mathfrak{r}-\varkappa)} \chi_{w}^{-\varkappa}
$$

Finally, by eq.(ב), $\mathrm{k}_{k}^{c}(t, x)$ too is a function of $w=t-x$ only and thus can be written as

$$
\begin{aligned}
\mathbb{k}_{k}^{c}(w) & =(R-\mathfrak{r}) \frac{1-\alpha}{k}\left(\frac{k}{1-\alpha}\left(h_{-\varkappa} \star \bar{Q}\right)(w)-\frac{(1-\alpha) k(R-\mathfrak{r}) \sigma v(\mathfrak{r}) \Phi(-\varkappa)}{\alpha \Phi(\mathfrak{r}-\varkappa)} \chi_{w}^{-\varkappa}\right) \\
& =(R-\mathfrak{r})\left(\left(h_{-\varkappa} \star \bar{Q}\right)(w)-C \sigma v(\mathfrak{r}) \chi_{w}^{-\varkappa}\right)
\end{aligned}
$$

Point iii: by the previous point $H=h_{-\varkappa} \star \bar{Q}-W$, where $W=C \sigma \psi_{0} \chi^{-\varkappa}$. Also, by lemma $1, \delta c=\mathbb{k}_{k}^{c} \star \delta k$. Recall also by the same lemma $\delta i=\delta y-\delta c$.

Since $i \mapsto \tilde{k}$ has derivative $\delta \tilde{k}=\xi_{R} \star \delta i$, so, with $\epsilon_{0}$ the unit mass at $0, \delta \tilde{k}=$ $(R-\mathfrak{r})\left(\epsilon_{0}-H\right) \star \xi_{R} \star \delta k$, i.e., $\frac{\partial \Upsilon}{\partial k}$ is indeed a convolution operator with kernel $\mathbb{k}_{k}^{k}=(R-\mathfrak{r}) \xi_{R} \star\left(\epsilon_{0}-H\right)$. Note $\mathbb{k}_{k}^{k}(t-x)=\mathrm{k}_{k}^{k}(t, x)$ (from lemma 1) at a BGE. $\mathbb{k}_{k}^{k} \in L_{1}^{\lambda} \forall \lambda<R$ since $\xi_{R}$ is so and $\epsilon_{0}-H$ has compact support. Finally, taking FT's, $\widehat{\mathbb{k}}_{k}^{k}=\frac{R-\mathfrak{r}}{R-\mathrm{i} \omega}(1-\widehat{H})$.

Observe that, for any $Q, \widehat{\bar{Q}}=\overline{\widehat{Q}}$, so $\widehat{H}=\widehat{h}_{-\varkappa} \overline{\widehat{Q}}-\widehat{W}$.

Now $\widehat{h}_{-\varkappa}(\omega)=\Phi(-\varkappa+\mathrm{i} \omega), \widehat{\phi}_{\mathfrak{r}}(-\omega)=v(\mathfrak{r}-\mathrm{i} \omega)$, and $\psi_{0}=v(\mathfrak{r})$. Hence representation (2), and, by direct computation, formula (5).

Point iv. We first show that $h_{-\varkappa}, Q$ and $W$ are jointly continuous, using the \|\|$_{p}$ topology for $h_{-\varkappa}$ and $W$ and weak* topology for $Q$. For $h_{-\varkappa}$ note that for any converging sequence in $\wp \times \mathbb{R}^{2}$, with limit $\varkappa_{0}, h_{-\varkappa}(s)$ converges uniformly to its limit $\mathbb{1}_{0 \leq s \leq 1} e^{-\varkappa_{0} s}$. The coefficients in the definitions of $Q$ and $W$, i.e., $B, \sigma$, $\Phi(-\varkappa), C, \bar{\psi}_{0}$ are clearly continuous in the parameters and $\mathfrak{r}$, as for any point in $G$ we have $\Phi(-\varkappa)>0,0<\alpha<1$ and $R>\mathfrak{r}$ (by cor. 3.i), so $B>0$. The conclusion

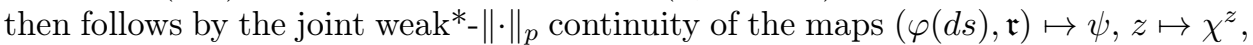
and the weak*-weak* continuity of $(\varphi(d s), \mathfrak{r}) \mapsto \phi_{\mathfrak{r}}(d s)$ on $G$. Next note that the 
map $h_{-\varkappa}, Q \mapsto h_{-\varkappa} \star \bar{Q}$ is $\|\cdot\|_{p^{-}}$continuous with the weak* topology on $Q$ and the $\|\cdot\|_{p}$ topology on $h_{-\varkappa}$ (cf. remark 1.i). ${ }^{20}$

6.3. Generic invertibility. So, now the task of identifying points on the equilibrium graph where the derivative is non-invertible reduces to finding the points where the Fourier transform of convolution kernel $\widehat{\mathbb{k}}_{k}^{k}$ (from lemma 2) of the derivative $\partial \Upsilon / \partial k$ at a BGE returns 1 for some parameter $\omega \in[0, \infty]$. The range of the Fourier transform is the set of complex numbers, $\mathbb{C}$, we are interested in "bad" points for which the real part of the transform, $\Re\left(\widehat{\mathbb{k}}_{k}^{k}\right)$, is unity, while the imaginary part, $\Im\left(\widehat{\mathbb{k}}_{k}^{k}\right)$, is zero for some $\omega$. Here is an illustration.

6.3.1. Illustrating spectra of $\frac{\partial \Upsilon}{\partial k}$ at a BGE. Figs. 5-12 show the spectra of the derivative of the equilibrium map, or the range of $\widehat{\mathbb{k}}_{k}^{k}$, for the four example economies as in fig. 1-4 where the life-time efficiency of labour is zero between 0 and $a$, unity between $a$ and $b$ and zero thereafter, i.e., $\varphi(s)=\frac{1}{b-a} \mathbb{1}_{[a, b]}(s)$.

First, note that spectrum is a line (not an "area") and even if it contains $(1,0)$ (in the complex plain), it is looks like a "rare" occasion: a slight change in parameters should shift the line away from the problematic $(1,0)$. This last claim, of course, has to be proven, and will be established in prop. 3 .

As an aside one could also notice that not all the spectra are in a unit circle, hence one should not expect $\Upsilon$ to be a contraction (in $k$ ) in general, thus making it difficult to find an equilibrium numerically (cf. the discussion in the introduction).

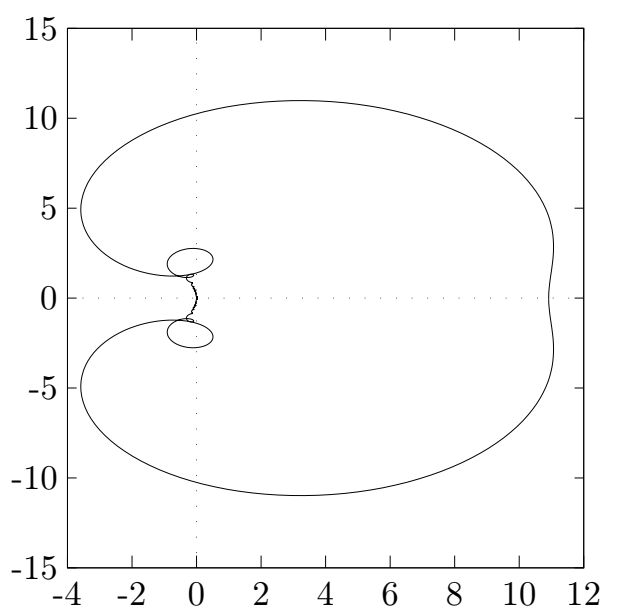

Figure 5. BGE of fig. 1 with $1-\frac{\mathfrak{r}}{R}=2$

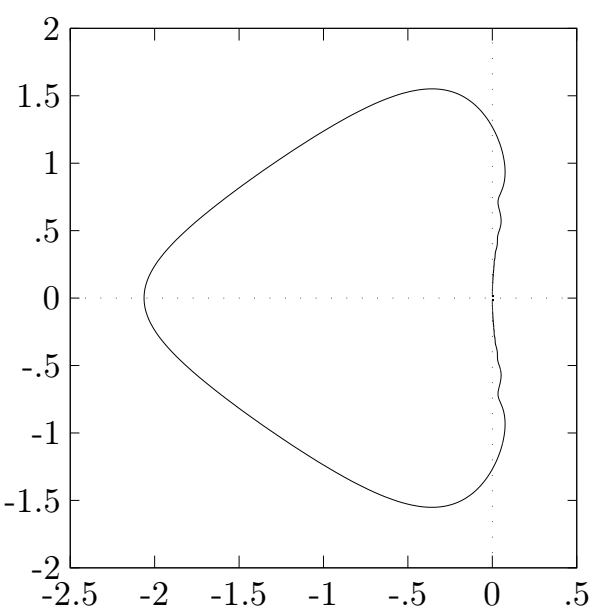

Figure 6. GRE of fig. 1 with $\alpha=.3$

6.3.2. $\frac{\partial F}{\partial k}$ is generically invertible at a BGE. Recall that one of the parameters of the economy is a life-cycle productivity measure $\varphi$, and so a negligible set of parameters (cf. def. 3 ) is defined for a fixed $\varphi$. The proof of the genericity claim (prop. 3) is based on a well-known property of analytic functions on $\mathbb{C}$ : their zeros are "isolated". An extension of this property for $\mathbb{C}^{n}$ case is in lemma 22 in the appendix and this is indeed all we need for our case. We will call a function defined on $\mathbb{C}^{n} \times \Delta([0,1])$ entire (analytic) if it is entire (analytic) on $\mathbb{C}^{n}$ for any fixed distribution $\varphi \in \Delta([0,1])$ and is jointly continuous in all the variables. If the function is initially defined on $\mathbb{R}^{n} \times \Delta([0,1])$, we will use its complex extension by analytic continuation.

\footnotetext{
${ }^{20}$ Note that for continuous distributions $\varphi$ the convergence of $H$ is uniform.
} 


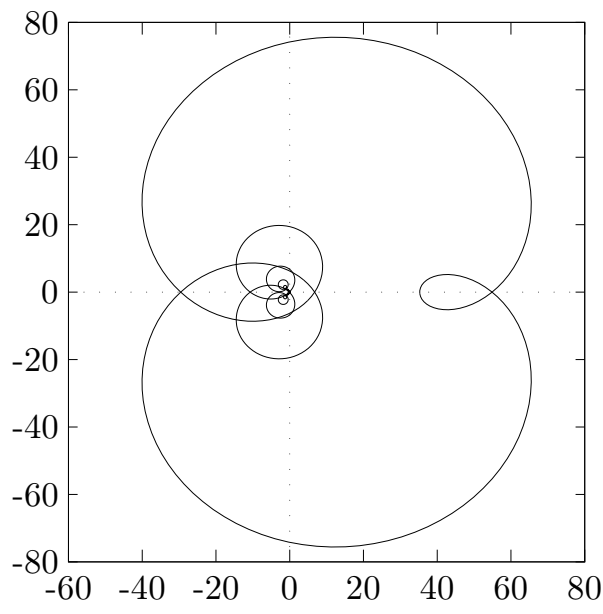

Figure 7. BGE of fig. 2 with $1-\frac{\mathfrak{r}}{R}=3$

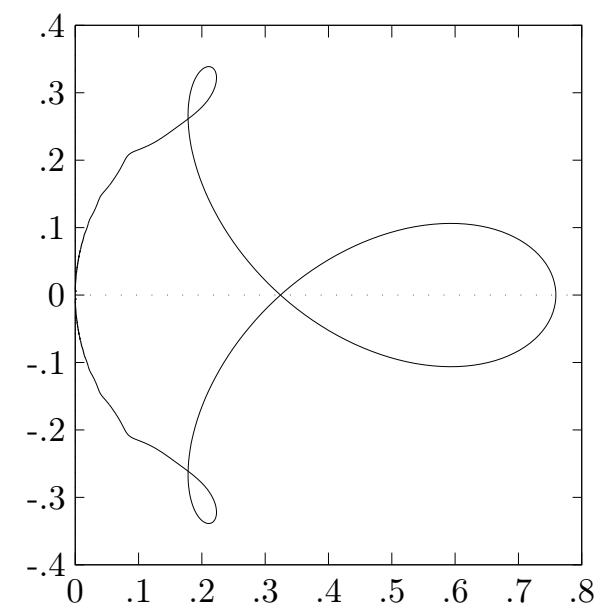

FiguRE 9. BGE of fig. 2 with $1-\frac{\mathfrak{r}}{R}=\frac{1}{2}$

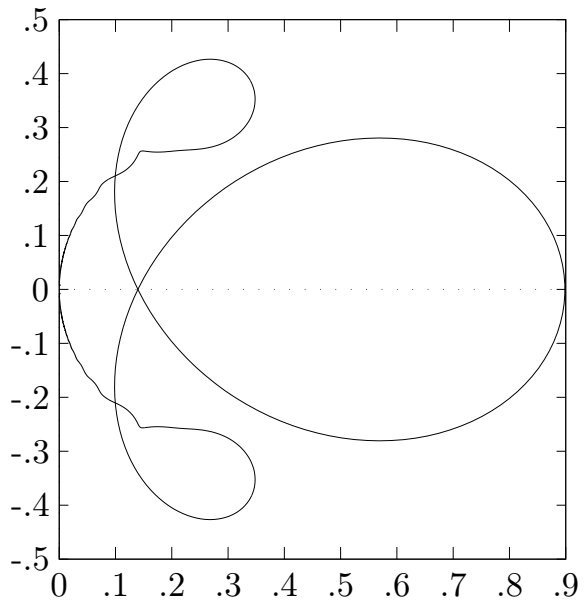

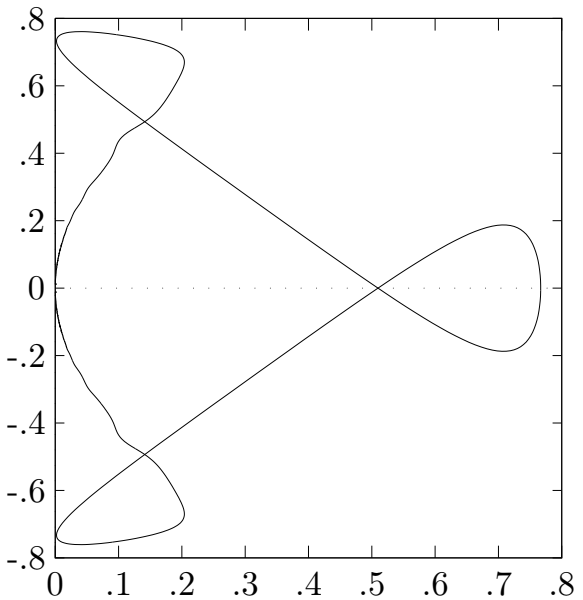

Figure 8. GRE of fig. 2 with $\alpha=.3$

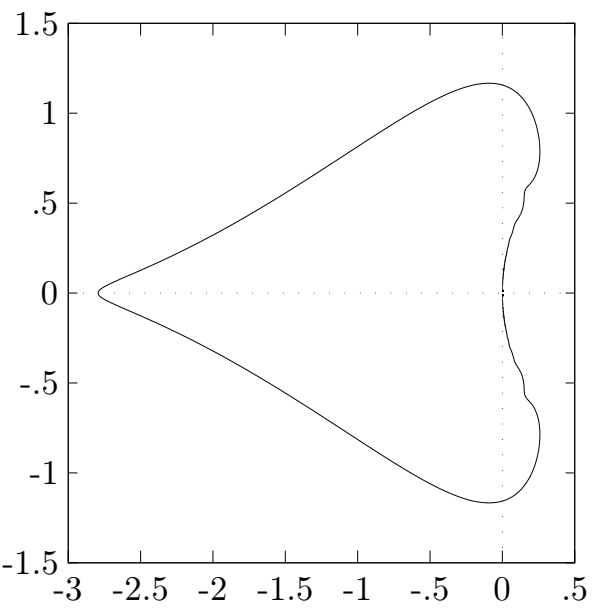

Figure 10. GRE of fig. 3 with $\alpha=.3$

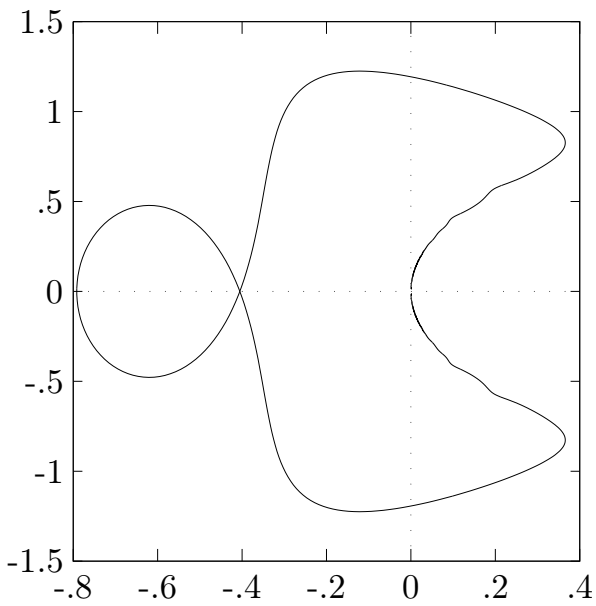

Figure 11. BGE of fig. 4 with $1-\frac{\mathfrak{r}}{R}=\frac{1}{2} \quad$ Figure 12 . GRE of fig. 4 with $\alpha=.3$ 
Proposition 3. The set $\mathcal{G} \subseteq \wp$ where 1 is not a value of $\widehat{\mathbb{k}}_{k}^{k}$ for any BGE is generic. Proof. Multiplying $1-\widehat{\mathbb{k}}_{k}^{k}=0$ by the non-null factor $\frac{R-\mathrm{i} \omega}{C(R-\mathfrak{r})}$ yields $(\mathfrak{r}-\mathrm{i} \omega)(D(\omega)+$ $\left.\frac{1}{C(R-\mathfrak{r})}\right)=0$, where $D(\omega) \stackrel{\text { def }}{=} \frac{\widehat{H}(\omega)}{C(\mathfrak{r}-\mathrm{i} \omega)}$; and hence the exceptional set $N \stackrel{\text { def }}{=} N_{0} \cup \tilde{N}$, where $N_{0}=\left\{g \mid \mathfrak{r}=0, \widehat{\mathbb{K}}_{k}^{k}(0)=1\right\}$, and $\tilde{N}=\left\{g \in G: \exists \omega: \frac{1}{C(\mathfrak{r}-R)}=D(\omega)\right\}$.

Claim 1. If $\mathfrak{r}=0$, the coefficient of $\sigma$ in $D$ equals

$$
\Xi(\omega, \eta)=\frac{1}{\omega^{2}+\eta^{2}}\left[1-\left(\frac{\frac{\sin \frac{\omega}{2}}{\frac{\omega}{2}}}{\frac{\sinh \frac{\eta}{2}}{\frac{\eta}{2}}}\right)^{2}\right]<\infty
$$

$0<\Xi(\omega, \eta) \leq \frac{1}{\omega^{2}+\eta^{2}}$ everywhere, and $\Xi(\omega, \eta) \sim \frac{1}{\omega^{2}+\eta^{2}}$ for $(\omega, \eta) \rightarrow \infty$

Proof. $\Xi(\omega, \eta) \underset{\omega, \eta \rightarrow 0}{\longrightarrow} \frac{1}{12}>0$. Now $\frac{\sin x}{x}$ (resp., $\frac{\sinh x}{x}$ ) is, for $x \neq 0$, in absolute value $<1$ (resp., $>1$ ), so for $(\omega, \eta) \neq(0,0)$, we also get $\Xi(\omega, \eta)>0$; so $\Xi>0$ everywhere. Remains thus only to show that $\Xi(\omega, \eta) \sim \frac{1}{\omega^{2}+\eta^{2}}$ for $(\omega, \eta) \rightarrow \infty$, which follows from $\frac{\sin \omega}{\omega} \underset{\omega \rightarrow \infty}{\longrightarrow} 0$ and $\frac{\sinh \eta}{\eta} \underset{\eta \rightarrow \infty}{\longrightarrow} \infty$.

$N_{0}=\left\{g \mid \mathfrak{r}=0, \frac{1}{C} \widehat{H}(0)=0\right\}$, and since by claim 1 the coefficient of $\sigma$ is $(\mathfrak{r}-\mathrm{i} \omega) \Xi=0$, and since $v(0)=\Psi(\eta, 0)=1$, suffices to express that, at $\omega=0$,

$$
B+\frac{1}{\mathrm{i} \omega}(\Psi(\eta,-\omega)-v(-\mathrm{i} \omega))=0
$$

By (4) in lemma 2 and the definition of $B$, this equation is equivalent to

$$
\widehat{\chi}^{-\eta}(0)-\widehat{\psi}(0)-\frac{\alpha}{(1-\alpha) R}=0
$$

Now $\widehat{\psi}(0)=\int_{0}^{1} s \varphi_{s} d s$ and $\widehat{\chi}^{-\eta}(0)=\frac{1}{\eta}-\frac{1}{e^{\eta}-1} ;$ thus $N_{0}$ is the set of zeros of

$$
W(R, \alpha, \eta, \varphi) \stackrel{\text { def }}{=} \frac{1}{\eta}-\frac{1}{e^{\eta}-1}-\int_{0}^{1} s \varphi_{s} d s-\frac{\alpha}{1-\alpha} \frac{1}{R}
$$

Since $\frac{1}{\eta}-\frac{1}{e^{\eta}-1}$ decreases from 1 to 0 , for any given $\varphi$, there is at most one value of any of the 3 parameters $R, \alpha, \eta$ that fits, given values of the other $2 .{ }^{21}$ So $N_{0}$ is closed and negligible.

To show that $\tilde{N}$ is negligible we establish, first, that the imaginary part of $D(\omega)$ has only a discrete set of zeros as a function of $\omega$ on $G \backslash G_{g}\left(G_{g}\right)$, this set depends on all parameters but $R(\sigma)$, and second that, for those $\omega, \frac{1}{C(\mathfrak{r}-R)}=\Re D(\omega)$ holds only for a discrete set of values $R(\sigma)$. Finally we show $N$ is closed.

Definition 9. For $g \in G, Z(g) \stackrel{\text { def }}{=}\{\omega \in \mathbb{R} \mid \Im D(\omega)=0\}$.

Step 1. The set of $g$ where $Z(g)$ is not discrete is negligible. On $G_{g}, Z(g)$ depends only on $(\eta, \alpha, R, \varphi)$, and on $G \backslash G_{g}, D$ (and hence $\left.Z(g)\right)$ only on $(\eta, \mathfrak{r}, \sigma, \varphi)$.

Proof of step 1. On $G_{g}$, since $\mathfrak{r}=0$, formula 5 of lemma 2 implies $\widehat{H}$ is purely imaginary (and so $\widehat{H} / \mathrm{i} \omega$ is real) iff $(B i \omega-1) v(-\mathrm{i} \omega)$ is real, i.e. iff $\frac{v(-\mathrm{i} \omega)}{1+B \mathrm{i} \omega}$ is real. But given $v(-\mathrm{i} \omega)=\widehat{\varphi}(-\omega)$, so the ratio is a Fourier transform of the convolution of $\bar{\varphi}$ with $B^{-1} \mathbb{1}_{t \leq 0} e^{B^{-1} t}$ (recall $B>0$ ). As $\varphi \supsetneqq 0$ has compact support, the support of the convolution is bounded on one side and unbounded on the other, so the convolution can not be even, hence its FT is not real.

Also, by formula 5 of lemma 2, the imaginary part of $D(\omega)=\frac{\widehat{H}(\omega)}{-C \mathrm{i} \omega}$ is independent of $\sigma$, hence its set of real zeros is so too, and it is discrete by lemma 22 .

Remains to prove the statement on $G \backslash G_{g}$.

\footnotetext{
${ }^{21}$ Since by cor. 3 , in a GRE $\frac{k}{c}=\frac{\alpha}{1-\alpha} \frac{1}{R}$, one can use [19, cor. 16] to show that if $W(R, \alpha, \eta, \varphi)$ is zero then the net assets are zero, $m=0$, in the GRE.
} 
Claim 2. (i) On $G \backslash G_{g}, B=\frac{\Phi(\mathfrak{r}-\varkappa)-\Phi(-\varkappa) v(\mathfrak{r})}{\mathfrak{r} \Phi(\mathfrak{r}-\varkappa)}$ and $C=\frac{\mathfrak{r} / B}{R \frac{\Phi(\mathfrak{r}-\varkappa)}{\Phi(-\varkappa)}-(R-\mathfrak{r}) v(\mathfrak{r})}$, so $D$ only depends on $(\eta, \mathfrak{r}, \sigma, \varphi)$.

(ii) $D$ is $J E$ on $\left(G \backslash G_{g}\right) \times \mathbb{R}$, where the last coordinate is $\omega$, and is the FT of the bounded measure $\frac{1}{C} H \star \ell_{\mathfrak{r}}$ on $[-1,1]$, where $\ell_{\mathfrak{r}}(x)=\operatorname{sign}(\mathfrak{r}) \mathbb{1}_{\mathfrak{r} x>0} e^{-\mathfrak{r} x}$.

Proof. Expressing $\alpha$ as a function of $\mathfrak{r} \neq 0$ using eq. 1 of cor. 3, get $\alpha=\frac{\Gamma(\mathfrak{r})}{1-\Gamma(\mathfrak{r})}$, where $\Gamma(\mathfrak{r}) \stackrel{\text { def }}{=} \frac{1-\digamma(\mathfrak{r}) v(\mathfrak{r})}{\mathfrak{r}}(R-\mathfrak{r})$, then the formulae in (i) follow by notation 6.2 defining $B$ and $C$. Thus the last clause, using also lemma 2.iii.

Point ii. To show that $D$ is entire in $\omega$, note that $H$ is a measure with bounded support, $[-1,1]$, so its Fourier transform is an entire function, i.e., limit of a power series (converging everywhere) with infinite radius of convergence. ${ }^{22}$ As $\widehat{H}$ is entire, the only possible pole of $D$ is at $-i \mathfrak{r}$, but a direct computation, using the formula for $B$ from point (i), shows that $\widehat{H}(-\mathrm{ir})$ is identically zero, so, using [19, lemma 16$]$ with $x=\omega$ and $y=-\mathrm{ir}, D$ is entire.

Since it is the FT of $\frac{1}{C} H \star \ell_{\mathfrak{r}}$ with $\ell_{\mathfrak{r}}(x)=\operatorname{sign}(\mathfrak{r}) \mathbb{1}_{\mathfrak{r} x>0} e^{-\mathfrak{r} x}$, and since this convolution must be proportional to $\ell_{\mathfrak{r}}$ outside $[-1,1]$, it follows that the proportionality factor must be 0 , else the FT would have a pole at $-\mathrm{ir}$. Thus this convolution is carried by $[-1,1]$.

Since $D(\omega)$ is the FT of this convolution, the joint continuity follows from the same property for $H$ (point (iv) of lemma 2 ) and $\ell_{\mathfrak{r}}$.

To establish joint analyticity, note that for any point in $G \backslash G_{g}, \mathfrak{r} \neq 0$, so $\mathfrak{r}-\mathrm{i} \omega \neq 0$. Given equation (5) for $\frac{\widehat{H}}{C}$ in lemma 2 , possible poles are at $\omega=0, \varkappa=2 k \pi$ i for $k \neq 0$ and $\varkappa-\mathfrak{r}=2 k \pi \mathrm{i}$ also for $k \neq 0$. The latter two are far away from $G \backslash G_{g}$, where $\varkappa$ and $\mathfrak{r}$ are real, so remains to prove joint analyticity of $\frac{\widehat{H}}{C}$ at $\omega=0$.

$$
\widehat{H}(\omega) / C=\frac{1}{\mathrm{i} \omega}(\Psi(\varkappa, \omega)((B \mathrm{i} \omega-1) v(\mathfrak{r}-\mathrm{i} \omega)+(1-\sigma) v(\mathfrak{r}) \Psi(\varkappa-\mathfrak{r},-\omega))+v(\mathfrak{r}) \sigma)
$$

Since $B$ and its coefficient are clearly analytic, it suffices to concentrate on $\frac{1}{\mathrm{i} \omega}(\Psi(\varkappa, \omega)((1-\sigma) v(\mathfrak{r}) \Psi(\varkappa-\mathfrak{r},-\omega)-v(\mathfrak{r}-\mathrm{i} \omega))+v(\mathfrak{r}) \sigma)$, which equals $-\Psi(\varkappa, \omega)(1-$ $\sigma) \tilde{\Psi}(\varkappa-\mathfrak{r},-\omega) v(\mathfrak{r})-\Psi(\varkappa, \omega)(1-\sigma) V-\sigma v(\mathfrak{r}-\mathrm{i} \omega) \tilde{\Psi}(\varkappa, \omega)-\sigma V$, with $\tilde{\Psi}(x, y)=$ $\frac{\Psi(x, y)-1}{\mathrm{i} y}, V=\frac{v(\mathfrak{r}-\mathrm{i} \omega)-v(\mathfrak{r})}{\mathrm{i} \omega}$.

So we need that $V$ and $\tilde{\Psi}(\varkappa, \omega)$ are JA at $\omega=0 . V=-\int_{0}^{1} e^{\mathfrak{r} t} t \Phi(-\mathrm{i} \omega t) \varphi(d t)$, and since the integrand is JE, the integral is so too. And $\tilde{\Psi}(\varkappa, \omega)$ is analytic by $[19$, lemma 16] except for poles at $\varkappa=2 k \pi \mathrm{i}, k \neq 0$ (i.e., the poles of $\Psi(-\varkappa, y)$ ).

Claim 3. $\left\{g \in G \backslash G_{g} \mid \Im D(\omega)=0 \forall \omega\right\}$ is negligible.

Proof. By claim 2.ii, $D(\omega)$ is the FT of a bounded measure. To show that $\Im D \neq 0$ it is sufficient to show that the derivative at zero is distinct from zero. Indeed, for a FT of a positive measure the real part is maximised at $\omega=0$, so the derivative at zero has zero real part. This conclusion is preserved for sums and differences of any positive measures, and thus for an arbitrary measure.

Then to prove the claim it is sufficient to show that $\left(\frac{d}{d \omega} D\right)(0)$ is distinct from zero for all but a negligible set of parameters. Given representation 2 of $\widehat{H}$ in lemma 2 , it is affine in $\sigma$ when expressed in terms of $\varphi, \varkappa, \mathfrak{r}, \sigma$ and so $\left(\frac{d}{d \omega} D\right)(0)$ is so too.

It remains to show then that the coefficient of $\sigma$ in $\left(\frac{d}{d \omega} D\right)(0)$ is zero for a negligible set of $(\varphi, \varkappa, \mathfrak{r})$. Let $A \stackrel{\text { def }}{=} \frac{\mathfrak{r}^{2}}{v(\mathfrak{r}) \mathrm{i}}\left(\frac{d^{2}}{d \omega d \sigma} D\right)_{\mid \omega=0}$ : since $\mathfrak{r}^{2} / v(\mathfrak{r})>0$ on $G \backslash G_{g}$, it suffices by lemma 22 to show that $A$ is JA and is not identically zero.

Given $D$ is JA on $G \backslash G_{g}$ by claim $2,\left(\frac{d}{d \omega} D\right)(0)$ is so too. Hence it is so for $\sigma=0$, then the JA property of the coefficient of $\sigma$, and therefore that of $A$ follows. Using

\footnotetext{
${ }^{22}$ Indeed, as the exponential function is entire, the series $\sum_{n} \frac{(z t)^{n}}{n !}$ converges everywhere, so by the Lebesgue's dominated convergence theorem $\widehat{H}$ is $\int e^{z t} H(t) d t=\sum_{n} \frac{z^{n}}{n !} \int t^{n} H(t) d t$, i.e., a power series in $z$ with infinite radius of convergence, $z \in \mathbb{C}$.
} 
again representation 2 of $\widehat{H}$ in lemma $2, A=\left.\mathfrak{r}^{2} \frac{d}{d \omega}\left(\frac{\Psi(\varkappa, \omega) \widehat{\chi}^{\mathfrak{r}-\varkappa}(-\omega)-\widehat{\chi}^{-\varkappa}(\omega)}{\mathrm{i}(\mathfrak{r}-\mathrm{i} \omega)}\right)\right|_{\omega=0}$. So $A(\mathfrak{r}, \varkappa)=\mathfrak{r} \int\left(\left(\frac{\Phi(\varkappa)-1}{\varkappa \Phi(\varkappa)}-t\right) \chi^{\mathfrak{r}-\varkappa}(t)-t \chi^{-\varkappa}(t)\right) d t+\int\left(\chi^{\mathfrak{r}-\varkappa}(t)-\chi^{-\varkappa}(t)\right) d t$. It is not identically zero since, given the identities, $\int \chi^{z}(t) d t=\frac{\Phi(z)-1}{z \Phi(z)}$ and $\int t \chi^{z}(t) d t=$ $\frac{z-2+2 \Phi(-z)}{2 z^{2} \Phi(-z)}, A(1,0)=\frac{e-4}{3(e-1)} \neq 0$.

Claim 4. The subset of $G_{b}$ where $Z(g)$ is not discrete is negligible, in addition, on $G \backslash G_{g} Z(g)$ is independent of $R$.

Proof. Given the representation of $B$ in claim 2.i, $D(\omega)$ is independent of $R$. In view of lemma 22 , given $\Im D$ is real-analytic for real arguments, this implies that the set of zeros of $\Im D(\omega)$ is discrete and is independent of $R$.

This finishes the proof of step 1 .

Step 2. $N$ is negligible in $G$.

Proof. Since $N_{0}$ is negligible, suffices to prove this for $\tilde{N}$. Partition $\tilde{N}$ into two sets: $\tilde{N}^{g}=\tilde{N} \cap G_{g}$ and its complement, $\tilde{N}^{b}$.

For $\tilde{N}^{g}$, given the definition of the exceptional set and the previous step, it suffices to verify that for any $\omega$ in the countable set $Z(g)$ there exists at most one value of $\sigma$ for which the real part of $D$ is equal to $-C R$. This is because $C=\frac{(1-\alpha)^{2} R}{\alpha}$ and $D=\Xi \sigma+$ const with $\Xi>0$ by claim 1 .

Note $\tilde{N}^{b}=\tilde{N}_{1} \cup \tilde{N}_{2}$, where $\tilde{N}_{1}=\left\{g \in G \backslash G_{g} \mid Z(g)\right.$ is not discrete $\}$ and $\tilde{N}_{2}=\left\{g \in G \backslash G_{g} \mid Z(g)\right.$ is discrete, $\left.\exists \omega \in Z(g): \Re D(\omega)=\frac{1}{C(\mathfrak{r}-R)}\right\}$. By step 1, $\tilde{N}_{1}$ is negligible. By claim 2.i, $\frac{1}{C(\mathfrak{r}-R)}=\frac{B}{\mathfrak{r}}\left(\frac{R}{\mathfrak{r}-R} \frac{\Phi(\mathfrak{r}-\varkappa)}{\Phi(-\varkappa)}-v(\mathfrak{r})\right)$, where $B$ is independent of $R$, and, recall, $B$ and $\mathfrak{r} \neq 0$. By step $1, \Re D(\omega)$ does not change with $R$, so there is at most one value of $R$ that satisfies the equality for every $\omega \in Z(g)$. Since $Z(g)$ is discrete, there are at most countably many values of $R$ that satisfy the equality, so $\tilde{N}_{2}$ is negligible.

Step 3. $N$ is closed in $G$.

Proof. Given the previous steps, it remains to show that $N$ is closed. Consider a sequence $g_{n} \in N$ with $g_{n} \rightarrow g_{0}$. Choose corresponding $\omega_{n}$ with $\widehat{\mathbb{K}}_{k}^{k}\left(\omega_{n}, g_{n}\right)=1$. Since $\|\widehat{H}\|$ is bounded on the sequence $g_{n}$ by lemma 2 iv used with $p=1$, and since $R-\mathfrak{r}$ is obviously bounded on the sequence, $\exists K: 1=\left\|\widehat{\mathbb{k}}_{k}^{k}\left(\omega_{n}, g_{n}\right)\right\| \leq \frac{K}{\left\|R_{n}-\mathrm{i} \omega_{n}\right\|}$, so $\omega_{n}$ is bounded. Thus, extracting a convergent subsequence, one can assume $\omega_{n} \rightarrow \omega_{0}$.

By lemma 2.iv, the map $H: G \rightarrow L_{1}$ is continuous, so, composing with FT: $L_{1} \rightarrow$ $C_{\infty}$ (by remark 1.ii), the composite map $\widehat{H}: G \rightarrow C_{\infty}$ is also continuous; hence the joint continuity of $\widehat{H}$ in $(\omega, g)$. Given $R>0, R-\mathrm{i} \omega \neq 0$, so $\widehat{\mathbb{k}}_{k}^{k}$ is also jointly continuous in $(\omega, g)$. This implies then $\widehat{\mathbb{k}}_{k}^{k}\left(\omega_{0}, g_{0}\right)=1$, so $g_{0} \in N$.

To complete the proof of the proposition observe that $G$ is a countable union of compact sets, the intersection of $N$ with each of those is compact and negligible by the previous steps and its projection onto $\wp$, i.e., the set of exceptional parameters, is compact. Remains to show this projection is negligible. This is obvious for $N \cap G_{g}$, since there the projection is basically the identity map. And on the complement, Fubini's theorem ensures that, outside a negligible set of $(R, \eta, \sigma, \varphi)$, the set of exceptional values of $\mathfrak{r}$ is negligible. For fixed $(R, \eta, \sigma, \varphi)$, our projection basically maps $\mathfrak{r}$ to $\alpha$, as in the equilibrium graphs (cf. figures 1-4), and this map is $C^{1}$, thus preserves negligible sets.

Remark 6 . We reduced the problem to show that negligibility is preserved when going from the equilibrium graph to the parameter space to the (trivial) 1-dimensional version of a statement that a $C^{1}$ map from $\mathbb{R}^{n}$ to $\mathbb{R}^{n}$ preserves negligibility (or, more 
generally, replacing $\mathbb{R}^{n}$ above by a $n$-dimensional manifolds with boundary, the first one being a $K_{\sigma}$ ). Such a statement seems easily provable from Sard's theorem and the implicit function theorem; we just didn't find the right reference yet.

It is such a statement that would be the right tool to handle the above problem in general. It is also the one (even its 1-dimensional version) that shows that neglecting above the difference between the equilibrium graph including the $y$ coordinate (as defined) and the graph without it (as used) is immaterial.

Remark 7. On the other hand, our technique to prove genericity relied on the fact that the (discrete) set of FT parameters $\omega$ that make the imaginary part of $\widehat{\mathbb{k}}_{k}^{k}$ zero did not depend on one of the parameters $(R)$, while the second condition (that the real part of $\widehat{\mathbb{k}}_{k}^{k}$ is unity) was satisfied for a finite set of $\omega$ for any given $R$. Thus, the specification of the model is deeply embedded in this part of the argument.

\section{Speed of CONVERGEnCE}

7.1. Establishing the speed of convergence. We assume here to deal with generic equilibria, and investigate the speed of convergence to 0 of the kernel of $\left(\frac{\partial F}{\partial k}\right)^{-1}$, which will later be seen to be also the speed of convergence of perturbed equilibria back to the original equilibrium, cf. rem. 12 .

Next corollary redefines the interval $\Lambda$ : its endpoints indicate the speed of convergence which, by corollary 4 of prop. 2 , can be traced back to the primitives of the model for a given choice of a BGE. We want the kernel $\mathbb{k}_{k}^{k}$ to be invertible: not only in $L_{1}$ but also in $L_{1}^{\lambda}$. So, we can ask what is the $\Lambda$ such that $e^{\lambda t} \mathbb{k}_{k}^{k}(t)$ invertible for $\lambda \in \Lambda$ ? We know by prop. 3 that for a generic point in the equilibrium graph, $\lambda=0$ is in that set. Corollary 4 establishes that the set is an interval (including zero). The upper bound of $\Lambda$ is the highest $\lambda$ for which $e^{\lambda t} \mathbb{k}_{k}^{k}(t)$ is not invertible, i.e., when its Fourier transform returns unity. But Fourier transform of $e^{\lambda t} \mathbb{K}_{k}^{k}(t)$ is a Laplace transform $(\mathcal{L})$ of the original kernel, $\mathbb{k}_{k}^{k}$. So, to rephrase, in order to find the upper bound of $\Lambda$ we have to find the root $z=\lambda+i \omega \in \mathbb{C}$ of the equation $\left(\mathcal{L} \mathbb{k}_{k}^{k}\right)(z)=1$ with the lowest real part $\lambda$. And to find the lower bound of $\Lambda$ we need the highest negative such $\lambda$. Since we also need $\mathbb{k}_{k}^{k} \in L_{1}^{\lambda}$, the upper bound of the interval should, in general, be below $R$ (cf. lemma 2.iii).

Corollary 4. For $z \in \mathbb{C}$ let $b(z) \stackrel{\text { def }}{=} \int_{-1}^{1} e^{z t} \mathbb{k}_{k}^{k}(t) d t\left(=\mathcal{L}\left(\mathbb{k}_{k}^{k}\right)(z)\right)$ with $\mathbb{k}_{k}^{k}$ from lemma 2.iv. the set $D=\{\Re(z) \mid b(z)=1\}$ is closed in $\mathbb{R}$ and discrete. Generically $0 \notin D$. Let then $\Lambda$ be the connected component of 0 in $\mathbb{R} \backslash D$ if $\mathcal{L}\left(\mathbb{k}_{k}^{c}\right)(R)=R-\mathfrak{r}$ and in $\mathbb{R} \backslash(D \cup\{R\})$ else. Then $\frac{\partial F}{\partial k}$ has as inverse in $\bigcap_{\Lambda} \mathcal{W}^{\lambda}$ a convolution operator $g-\mathbf{1}$, and $\int e^{z t} g(t) d t=1+\frac{1}{b(z)-1}$ for $\Re(z) \in \Lambda$.

Proof. For $z \in \mathbb{C}$ let $G(z)=\int_{-1}^{1} e^{z t} H(t) d t$, with $H=\frac{1}{R-\mathfrak{r}} \mathbb{k}_{k}^{c}$ from lemma 2.iv. Since $H$ has support in $[-1,1], G$ is entire and $|G(z)| \leq\|H\|_{1} e^{|\Re(z)|}$, so for $\Re(z)$ bounded $\left\{z \mid G(z)=\frac{z-\mathfrak{r}}{R-\mathfrak{r}}\right\}$ must be compact. By analyticity, it is discrete, hence finite. So its set of real parts, being finite on every bounded set, is closed in $\mathbb{R}$ and discrete. This remains thus true for $D$, which differs from it at most by the removal of 1 point, $R$, because of the division by $R-z$. By prop. 3, generically $0 \notin D$.

The rest follows now by applying prop. 2 , with $f=\mathbb{k}_{k}^{k}\left(=\frac{\partial \Upsilon}{\partial k}\right)$ since $\frac{\partial F}{\partial k}=\mathbb{k}_{k}^{k}-\mathbf{1}$ (prop. 1). The convolution $\mathbb{k}_{k}^{k}$ (lemma 2.iii) of $(R-\mathfrak{r})\left(\epsilon_{0}-H\right)$ (carried by $\left.[-1,1]\right)$ with $\mathbb{1}_{t \geq 0} e^{-R t}$, equals $(R-\mathfrak{r})(1-G(R)) e^{-R t}$ for $t \geq 1$, where $R-\mathfrak{r}>0$. So, if $G(R)=1$ (and so $\mathcal{L}\left(\mathbb{k}_{k}^{c}\right)(R)=R-\mathfrak{r}$ ), $\mathbb{k}_{k}^{k}$ itself has support in $[-1,1]$, and $\widehat{\mathbb{k}}_{k}^{k}$ is entire. Thus, in this case, $\mathbb{k}_{k}^{k} \in L_{1}^{\lambda} \forall \lambda: J=\mathbb{R}$. Else, $\mathbb{k}_{k}^{k} \in L_{1}^{\lambda}$ iff $\left.\lambda<R: J=\right]-\infty, R$. Also $\left(\mathcal{L} \mathbb{k}_{k}^{k}\right)(z)=\frac{R-\mathfrak{r}}{R-z}(1-G(z))$, by analytic continuation, since by lemma 2 .iii $\left(\widehat{\mathbb{k}}_{k}^{k}\right)(\mathrm{i} \omega)$ is given by this formula, and since $b=\mathcal{L} \mathbb{k}_{k}^{k}$ is analytic by prop. 2 and $G$ entire as seen above. 
Corollary 5. The convolution operator $g-\mathbf{1}$ is the inverse of $\frac{\partial F}{\partial k}$ in $\mathbb{L}^{\Lambda}\left(C_{\infty}, C_{\infty}\right)$.

Proof. By cor. 4, it is the inverse in $\bigcap_{\Lambda} \mathcal{W}^{\lambda}$. By lemma 6.iii (in app. A), its operator norm on $L_{p, q}^{\lambda}$ is $\leq\|g-\mathbf{1}\|^{\lambda}$, its norm in $\mathcal{W}^{\lambda}$. Thus $\|g-\mathbf{1}\|_{J} \leq \sup _{\lambda \in J}\|g-\mathbf{1}\|^{\lambda}$. This is finite since $\|g-\mathbf{1}\|^{\lambda}$ is finite and convex.

Corollary 6. Let $\Lambda=] \lambda_{-}, \lambda_{+}\left[\right.$. Then $\left|\lambda_{-}\right|$and $\lambda_{+}$are resp. the speeds of convergence of $g$ to 0 at $-\infty$ and at $+\infty$, in the sense that $\Lambda=\left\{\lambda \mid g \in L_{1}^{\lambda}\right\}$, except that the speed at $+\infty$ may be faster when $\lambda_{+} \notin D \cup\{+\infty\}$.

Proof. $\subseteq$ follows from cor. 4 ; $\supseteq$ from cor. 1 if $G(R)=1$ or $\lambda_{+}<R$.

Since $\lambda_{+} \notin D \cup\{+\infty\}$ means $G(R) \neq 1, \lambda_{+}=R \notin D$, remains thus only to prove $\supseteq$ when $G(R) \neq 1, \lambda_{+}=R \in D$. Then, if, to the contrary, $g \in L_{1}^{R}, \int e^{z t} g(t) d t$ would be continuous on $\{z \mid 0 \leq \Re(z) \leq R\}$, by the dominated convergence theorem. $G$ being continuous, this implies the formula for $\int e^{z t} g(t) d t$ (cor. 4 ), being valid for $\Re(z) \in[0, R[$, holds for $\Re(z) \in[0, R]$, since no division of 0 by 0 can occur, by $G(R) \neq 1$.

In particular, $\int e^{(R+\mathrm{i} \omega) t} g(t) d t=1$ iff $\omega=0$, and, for $\Re(z) \in[0, R],\left|\int e^{z t} g(t) d t\right|<$ $\infty$ implies $S(z) \neq 0: D$ is disjoint from $[0, R]$, contradiction.

Remark 8 . The main result implies that the same holds true for the speeds of convergence of perturbed equilibria towards the original equilibrium at $-\infty$ and $+\infty$.

7.2. Illustrating the speed of convergence. Figs. 13-20 illustrate, for the example economies of section 6.3.1, the rates of convergence $\lambda_{-}$(below the $x$-axis) and $\lambda_{+}$(above the $x$-axis) as a function of $\alpha$ for the GRE and of $x=1-\frac{\mathfrak{r}}{R}\left(=\frac{\alpha Y}{I}\right)$ for the other BGE. $2 \mathrm{~L}$ and $2 \mathrm{H}$ in the captions of fig. 16 and 18 refer to the low and high intervals of $x$ for which BGE exists.

We know from claim 2.ii (cf. also its proof if desired) that $z=\mathfrak{r}$ is always a root for BGE; it is the straight line passing through $(0, R)$ and $(1,0)$, and segments of it are visible e.g. in fig. 16 and 20. Another segment of it is $\lambda_{-}$after the critical point in fig. 18, which does't appear since being $<-60$ it would fall far off the page. Same for the whole of $\lambda_{-}$in fig. 14 .

Critical points correspond to the intersections with the $x$-axis. All but one of them are "trivial", in the sense that they correspond to real roots, which one knew must be there just by looking at the graphs of figs. [19, 1-4]: the local minima and maxima of the BGE curves in those graphs, and the intersection with the GRE line, as well as $\alpha=1$. The one exception is the critical point in fig. 18 ( $x=6.768475$, $z= \pm 8.07776 \mathrm{i}$ ).

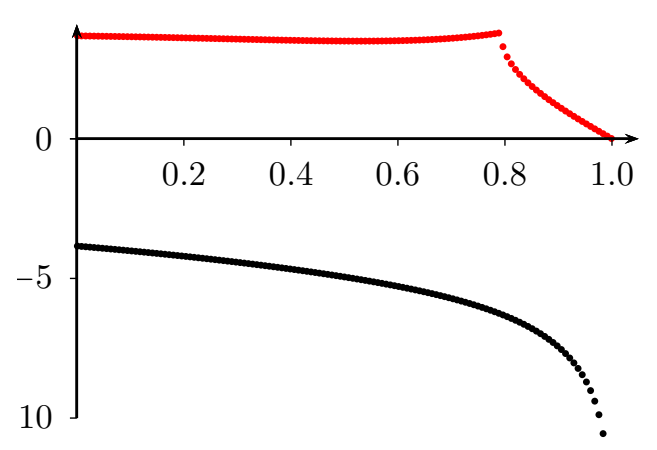

Figure $13 . \lambda_{+}$and $\lambda_{-}$; GRE of ex. 1.

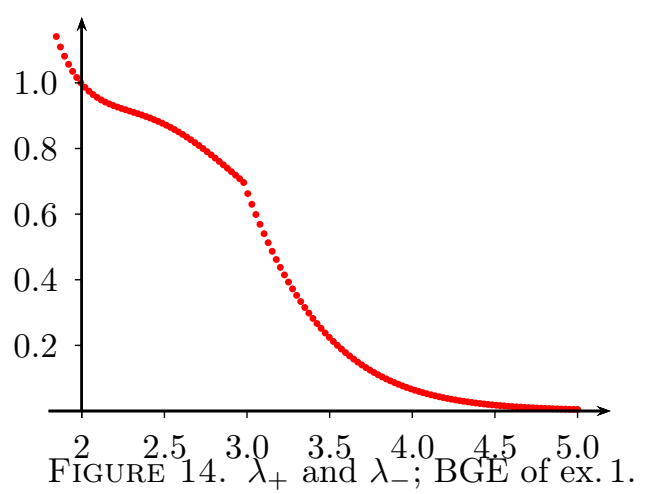


0

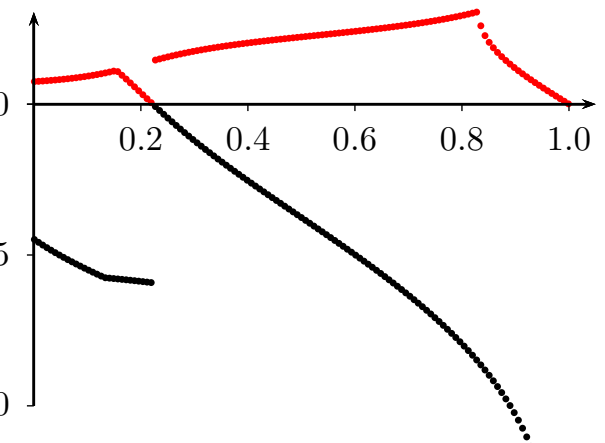

FIGURE 15. $\lambda_{+}$and $\lambda_{-}$; GRE of $\dot{\circ}^{\circ} \cdot 2$.

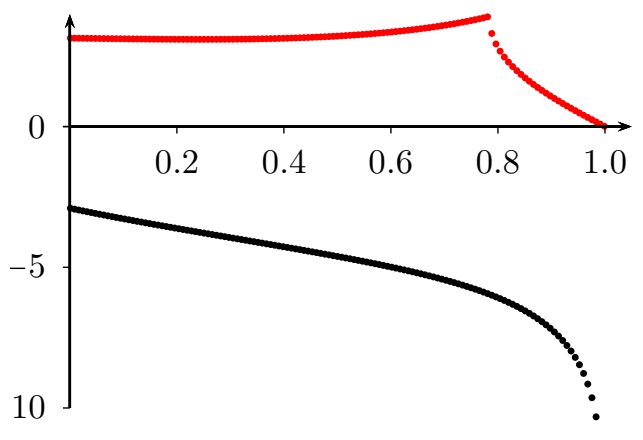

Figure 17. $\lambda_{+}$and $\lambda_{-}$; GRE of ex. 3 .

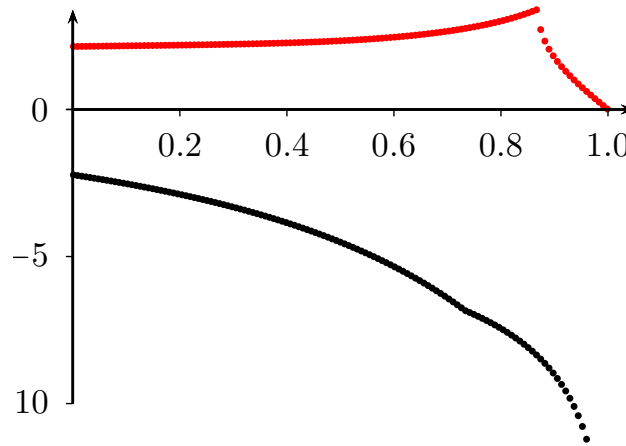

Figure 19. $\lambda_{+}$and $\lambda_{-}$; GRE of èx. 4.

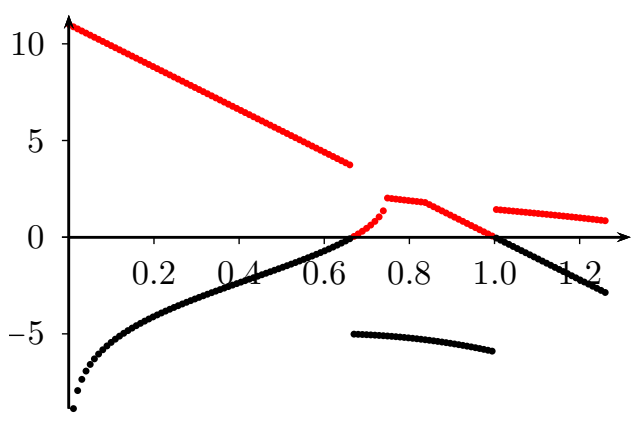

Figure $16 . \lambda_{+}$and $\lambda_{-}$; BGE of ex. 2L.

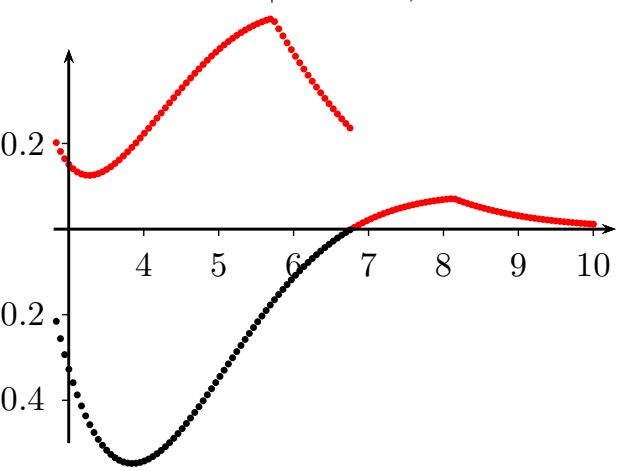

FigURE $18 . \lambda_{+}$and $\lambda_{-}$; BGE of ex. $2 \mathrm{H}$.

\section{USING THE IMPLICIT FUNCTION THEOREM}

The following lemma (3) could have been our main result establishing regularity and stability of solutions, and certainly can be viewed as such, however, we want to present the results in a simpler form (with easier metrics and using standard differentiability concepts). Besides, the other aim is to formulate the results independently of the fixed-point map used, ${ }^{23}$ a.o., to get correct bounds for each variable for its own sake - i.e., "to cover our tracks."

But first, let us use the implicit function theorem, thus combining the results we got up to this point.

Here we consider the solutions (defined in section 3.2) belonging to $P=C_{\infty}^{4} \times$ $L_{\infty, 1}$, where all the spaces are taken with their corresponding families (cf. def. 6). In what follows $\lambda_{+}$and $\lambda_{-}$are as defined in cor. 6 , and the generic set $\mathcal{G}$ - in prop. 3 .

\footnotetext{
${ }^{23}$ For a given equilibrium equation system: indeed the system could be represented, instead of a map from $k$ to $\tilde{k}$, as a map from $i$ to $\tilde{i}$, for example.
} 
Lemma 3. Everywhere in $\mathcal{G}, \exists \delta>0$ and an $\|\cdot\|_{\infty, 1}$ open ball $B$ s.t., for any $B G E$ $\varpi(0)$, there is for $E \in B$ a unique solution $\varpi(E) \in P$ with $\left\|\varpi_{k}(E)-\varpi_{k}(0)\right\| \leq \delta$. Further, $\forall \varepsilon>0 \exists$ an $\|\cdot\|_{\infty, 1}$ open ball $B_{\varepsilon} \subseteq B$ s.t., $\forall B G E \exists$ a compact interval $\Lambda^{\varepsilon} \subseteq \Lambda$ with $\max \left\{\min \left\{0, \lambda_{-}+\varepsilon\right\}, \frac{-1}{\varepsilon}\right\}$ and $\left(\lambda_{+}-\varepsilon\right)^{+}$in its interior, s.t., on $B_{\varepsilon}$ :

(i) $E \mapsto \varpi(E)$ is $S_{\left\{\Lambda^{\varepsilon}\right\}}^{1}$, and $E \mapsto \varpi^{\prime}(E)$ is Lipschitz to $\mathbb{L}_{\left\{\Lambda^{\varepsilon}\right\}}\left(L_{\infty, 1}, P\right)$.

(ii) $\exists K: \forall \lambda \in \Lambda^{\varepsilon}, \forall p,\left\|\varpi_{q}\left(E_{1}\right)-\varpi_{q}\left(E_{2}\right)\right\|_{p}^{\lambda} \leq K\left\|E_{1}-E_{2}\right\|_{p}^{\lambda}$ for any component $q$ of solution $\varpi$.

(iii) $\frac{\partial F}{\partial k}$ has at $\left(E, \varpi_{k}(E)\right)$ an inverse in $\mathbb{L}_{\left\{\Lambda^{\varepsilon}\right\}}\left(C_{\infty}, C_{\infty}\right)$. This is Lipschitz in $E$.

Proof. Suffices to do the proof for a fixed BGE, then to replace $\delta$ and $B_{\varepsilon}$ by their minimum over all (finitely many, [19, thm. 3]) BGE, then to decrease $B_{\varepsilon}$ again to fit the new $\delta$. Note that $\Lambda$ and thus $\Lambda^{\varepsilon}$ depend on the BGE.

By cor. $3, k^{*}>0$ in the BGE, so $O=\left\{k \mid \inf _{t} k_{t}>0\right\}$ is open in $C_{\infty}$ and contains $k^{*}$. By lemma 1.ii $F$ is $S^{1}$ on $O \times L_{\infty, 1}$, and by cor. $5, \frac{\partial F}{\partial k}$ is invertible at the BGE in $\mathbb{L}^{\Lambda}\left(C_{\infty}, C_{\infty}\right)$ on the generic set $\mathcal{G}$ of prop. 3. Thus [20, thm. 3] is applicable and yields an implicit function $\varpi_{k}$ s.t. i and iii hold. By lemma 1, i,c,y, r are $S^{1}$ functions of $k$ and $E$, so by [20, prop. 5] their compositions with $\varpi_{k}$ are also $S^{1}$ functions of $E$ : adding them as additional coordinates to $\varpi$ yields an $S^{1}$ map as stated, since ii follows by applying [20, prop. 3.iv] to further reduce $B_{\varepsilon}$.

Corollary 7. On $B_{\varepsilon}, \varpi_{k}$ is uniformly bounded away from 0 , and all $C_{\infty}$-components are uniformly bounded.

Proof. Since $B$ is bounded, ii implies (with $\lambda=0, p=\infty$ ) that the $C_{\infty}$-components are uniformly bounded. $\varpi_{\mathfrak{r}}$ being uniformly bounded implies by prop. 1.ii and vi that $\varpi_{k}$ is uniformly bounded away from 0 .

Remark 9. We could as well have viewed our fixed point map $\Upsilon$ e.g. as a map from $i$ to $\tilde{\imath}$, rather than from $k$ to $\tilde{k}$; basically everything still goes through in the same way. But one then obtains a weaker 'local uniqueness' result in lemma 3: it would then refer to a $\delta^{\prime}$ neighbourhood of $\varpi_{i}$, and that would be in $L_{\infty, 1}$. The map $i \rightarrow k$ is continuous and injective, but the inverse is not at all continuous, so our present result is definitely sharper (and simpler).

\section{Derivatives ARE GIVEN BY KERNELS}

We first show that the derivatives $\left(\varpi^{\prime}\right)$ are mostly given by properly behaving kernels, and s.t. the statement of the main result (relying on the implicit function theorem) is independent of the fixed-point map used (on $i$, on $k, \ldots$ ).

Definition 10. A kernel operator is a continuous linear map $A$ from $L_{\infty}\left(\mathbb{R}^{k}\right)$ to $C_{\infty}\left(\mathbb{R}^{n}\right)$ s.t., for Borel sets $B_{n} \searrow \emptyset, A\left(\mathbb{1}_{B_{n}}\right) \rightarrow 0$ pointwise.

Remark 10. In the language of dynamic systems, the expression of $\varpi^{\prime}$ by kernels $\mathrm{k}(x ; t, t-s)$ is equivalent to the full system of impulse-responses for all possible (small) impulses: $\mathrm{k}(x ; t, t-s)$, as a function of $x$, is the response to an impulse at $(t, s)$.

9.1. Kernel representation of $\left(\frac{\partial F}{\partial k}\right)^{-1}$ at a BGE. Since we want to formulate our main results in terms of a simpler norm (for the kernels) that expresses directly the exponential convergence aspect, we start by establishing such properties for the kernel of the inverse, $\left(\frac{\partial F}{\partial k}\right)^{-1}$. But first, we need to define the norms. ${ }^{24}$

\footnotetext{
${ }^{24}$ One of the norms has been already introduced in lemma 1.v, but we provide the definitions of both here for easier reference.
} 
Notation 9.1. $\psi_{\lambda, \lambda^{\prime}}(z)=e^{\lambda z}+e^{\lambda^{\prime} z}$, and for a compact interval $J, \psi_{J}=\psi_{\min J \text { max } J}$. For a kernel $\mu(x, d t),\|\mu\|_{J}^{C C}=\sup _{x} \int \psi_{J}(x-t)|\mu(x, d t)|,\left(=\sup _{x}\left(\psi_{J} \star|\mu(x, \cdot)|\right)(x)\right)$. For a kernel $\mathrm{k}(x ; t, s)$, let $\|\mathrm{k}\|_{J}^{L C}=\sup _{x} \operatorname{ess~sup~}_{s, t} \psi_{J}(x-t)|\mathrm{k}(x ; t, s)|$.

$\lambda_{-}^{\varepsilon}=\min \Lambda^{\varepsilon}$, and $\lambda_{+}^{\varepsilon}=\max \Lambda^{\varepsilon}$. $\psi_{\varepsilon}=\psi_{\Lambda^{\varepsilon}}$, for a kernel $\mu(x, d t),\|\mu\|_{\varepsilon}=\|\mu\|_{\Lambda^{\varepsilon}}^{C C}$, and for $\mathrm{h}(x, t),\|\mathrm{h}\|_{\varepsilon}=\|\mathrm{h}(x, t) d t\|_{\varepsilon}$.

Lemma 4. With $\mathrm{k}_{k}^{k}$ the kernel of $\frac{\partial \Upsilon}{\partial k}$ (lemma 1.iv), $\forall\left(E, \varpi_{k}(E)\right.$ ), $\exists$ a bounded $\mathrm{h}(x, t)=\mathrm{h}_{c}(x, t)-\alpha \frac{y_{t}}{k_{t}} \xi_{R}(x-t)$, where $h_{c}(x, t)$ is uniformly continuous in $x$ to $C_{\infty}$, s.t.:

(i) $E \mapsto \mathrm{h}_{(E)}$ is Lipschitz from $B_{\varepsilon}$ to $\|\cdot\|_{\Lambda^{\varepsilon}}^{L C}$.

(ii) $\mathrm{h}$ is the $d z$-a.e.-unique solution of $\mathrm{h}(x, z)+\mathrm{k}_{k}^{k}(x, z)=\int \mathrm{k}_{k}^{k}(x, t) \mathrm{h}(t, z) d t$ (dz-a.e.) s.t. $x \mapsto \mathrm{h}(x, z) d z$ is weak ${ }^{*}$-continuous and bounded. ${ }^{25}$

The same holds for the equation $\mathrm{h}(x, z)+\mathrm{k}_{k}^{k}(x, z)=\int \mathrm{h}(x, t) \mathrm{k}_{k}^{k}(t, z) d t$. Both equations hold everywhere, and the second then defines h everywhere.

(iii) $\mathrm{h}-\mathbf{1}$ is the inverse of $\mathrm{k}_{k}^{k}-\mathbf{1}$ in $\mathbb{L}_{\left\{\Lambda^{\varepsilon}\right\}}\left(C_{\infty}, C_{\infty}\right)$, in $\mathbb{L}_{\left\{\Lambda^{\varepsilon}\right\}}\left(L_{\infty, 1}, L_{\infty, 1}\right)$, and in all $\mathbb{L}\left(L_{p, q}^{\lambda}, L_{p_{f} q}^{\lambda}\right)$ and $\mathbb{L}\left(C_{p}^{\lambda}, C_{p}^{\lambda}\right)\left(\lambda \in \Lambda^{\varepsilon}\right)$.

For $\mu^{k}(x, d z) \stackrel{\text { def }}{=} \mathrm{h}(x, z) d z-\epsilon_{x},\left(\frac{\partial F}{\partial k}\right)^{-1}=\left[g \mapsto \int g(z) \mu(d z)\right]$.

Proof. iii and ii: The inverse $I$ of $\frac{\partial F}{\partial k}$ exists in $\mathbb{L}_{\left\{\Lambda^{\varepsilon}\right\}}\left(C_{\infty}, C_{\infty}\right)$ by lemma 3.iii. By lemma 1.iv-vi, $\frac{\partial \Upsilon}{\partial k}\left(=\frac{\partial F}{\partial k}+\mathbf{1}\right)$, at $\left(\varpi_{k}(E), E\right)$, is described by a bounded kernel $\mathrm{k}_{k}^{k} \in \mathbb{L}^{\Lambda}\left(L_{\infty, 1}, C_{\infty}\right)$, so $\left\|\mathrm{k}_{k}^{k}\right\|_{\Lambda^{\varepsilon}}^{L C}<\infty$. Thus $\mathrm{k}_{k}^{k}$ is continuous to $L_{1}$ : if $x_{n} \rightarrow x$, $\left\|\mathrm{k}_{k}^{k}\right\|_{\varepsilon}<\infty$ implies the tightness of the corresponding measures, and the boundedness of $\mathrm{k}_{k}^{k}$ allows to use the bounded convergence theorem on bounded sets; finally, a.e. convergence is clear from lemma 1 .iv-vi. $\mathrm{k}_{k}^{k}$ is also bounded to $L_{1}$, by $\left\|\mathrm{k}_{k}^{k}\right\|_{\Lambda^{\varepsilon}}^{L C}<$ $\infty$. Apply now lemma 7 to establish existence of the inverse and its properties.

By lemma $7, \mathrm{~h}$ is continuous and bounded to $L_{1}$, and $\mathrm{k}_{k}^{k}$ bounded by lemma 1.vi, so $G(x, z)=\int \mathrm{h}(x, t) \mathrm{k}_{k}^{k}(t, z) d t$ is bounded and continuous (using the continuity properties of $\mathrm{k}_{k}^{k}$ in lemma 1 .vi to get a.e. convergence). Define now h everywhere by $\mathrm{h}=-\mathrm{k}_{k}^{k}+G: \mathrm{h}(x, t)$ is bounded, and, by lemma $1 . \mathrm{iv}$, vi, equals a continuous function minus $\alpha \mathbb{1}_{t \leq x} e^{-R(x-t)} \frac{y_{t}}{k_{t}}$. Finally, the same argument as above for $G$ can now be used for $\int \mathrm{k}_{k}^{k}(x, t) \mathrm{h}(t, z) d t$ : it too is continuous, with this specification of $\mathrm{h}$, hence that equation too holds everywhere.

Denoting the continuous and discontinuous parts of $\mathrm{k}_{k}^{k}$ by $\mathrm{k}_{k}^{k c}$ and $\mathrm{k}_{k}^{k d}$, as in lemma $1, G(x, z)=\int\left(\mathrm{k}_{k}^{k c}(x, t)+\mathrm{k}_{k}^{k d}(x, t)\right) \mathrm{h}(t, z) d t$. Since $\frac{y_{t}}{k_{t}} \mathrm{~h}(t, z)$ is bounded, cor. 10 implies the term with $\mathrm{k}_{k}^{k d}$ is Lipschitz from $x$ to $C_{\infty}$. By lemma $1 . v i, \mathrm{k}_{k}^{k c}$ is so too, and being bounded by a constant times $\xi_{R}(x-t+1)$, it follows that it is uniformly continuous and bounded to $C_{1}$. Thus, h being bounded, $\int \mathrm{k}_{k}^{k c}(x, t) \mathrm{h}(t, z) d t$ is uniformly continuous from $x$ to $C_{\infty}$. Thus so is $G$ itself, and thus also $\mathrm{h}_{c}=-\mathrm{k}_{k}^{k c}+G$.

i: $\left\|\mu^{k}\right\|_{\varepsilon}=1+\|\mathrm{h}\|_{\varepsilon}$ and $\left\|\mu_{1}^{k}-\mu_{2}^{k}\right\|_{\varepsilon}=\left\|\mathrm{h}_{1}-\mathrm{h}_{2}\right\|_{\varepsilon}$; so by lemma 3.iii and prop. 5 $E \mapsto \mathrm{h}_{(E)}$ is Lipschitz to $\mathbb{L}_{\left\{\Lambda^{\varepsilon}\right\}}\left(C_{\infty}, C_{\infty}\right)$. Now the second equation, $\mathrm{h}=-\mathrm{k}_{k}^{k}+\mathrm{hok} k$, can be viewed as an operator equation, where in $\mathrm{h} \circ \mathrm{k}_{k}^{k}$ first $\mathrm{k}_{k}^{k}$ is applied to some $f \in L_{\infty, 1}$, next $\mathrm{h}$ is applied to the result. Indeed, Fubini applies, since, by shrinking a bit $\Lambda^{\varepsilon},|\mathrm{h}| \in \mathbb{L}_{\left\{\Lambda^{\varepsilon}\right\}}\left(C_{\infty}, C_{\infty}\right)$ by prop. 5 and $\left|\mathrm{k}_{k}^{k}\right| \in \mathbb{L}_{\left\{\Lambda^{\varepsilon}\right\}}\left(L_{\infty, 1}, C_{\infty}\right)$ by prop. 6 . Thus $\|\mathrm{h}\|_{\mathbb{L}_{\left\{\Lambda^{\varepsilon}\right\}}\left(L_{\infty, 1}, C_{\infty}\right)} \leq\left[1+\|\mathrm{h}\|_{\mathbb{L}_{\left\{\Lambda^{\varepsilon}\right\}}\left(C_{\infty}, C_{\infty}\right)}\right]\left\|\mathrm{k}_{k}^{k}\right\|_{\mathbb{L}_{\left\{\Lambda^{\varepsilon}\right\}}\left(L_{\infty, 1}, C_{\infty}\right)},<\infty$ by lemma 1.v: $\mathrm{h} \in \mathbb{L}_{\left\{\Lambda^{\varepsilon}\right\}}\left(L_{\infty, 1}, C_{\infty}\right)$. Similarly, going to differences in that equation, i.e., $\delta \mathrm{h}=$ $-\delta \mathrm{k}_{k}^{k}+\delta \mathrm{h} \circ\left(\mathrm{k}_{k}^{k}+\delta \mathrm{k}_{k}^{k}\right)+\mathrm{h} \circ \delta \mathrm{k}_{k}^{k}$, yields $\|\delta \mathrm{h}\|_{\mathbb{L}_{\left\{\Lambda^{\varepsilon}\right\}}\left(L_{\infty, 1}, C_{\infty}\right)} \leq\left\|\delta \mathrm{k}_{k}^{k}\right\|_{\mathbb{L}_{\left\{\Lambda^{\varepsilon}\right\}}\left(L_{\infty, 1}, C_{\infty}\right)}+$ $\|\delta \mathrm{h}\|_{\mathbb{L}_{\left\{\Lambda^{\varepsilon}\right\}}\left(C_{\infty}, C_{\infty}\right)}\left\|\mathrm{k}_{k}^{k}+\delta \mathrm{k}_{k}^{k}\right\|_{\mathbb{L}_{\left\{\Lambda^{\varepsilon}\right\}}\left(L_{\infty}, 1, C_{\infty}\right)}+\|\mathrm{h}\|_{\mathbb{L}_{\left\{\Lambda^{\varepsilon}\right\}}\left(C_{\infty}, C_{\infty}\right)}\left\|\delta \mathrm{k}_{k}^{k}\right\|_{\mathbb{L}_{\left\{\Lambda^{\varepsilon}\right\}}\left(L_{\infty}, 1, C_{\infty}\right)}$. By lemma 1.v, we can reduce $B_{\varepsilon}$ so that $E \mapsto \mathrm{k}_{k(E)}^{k}$ is Lipschitz from $B_{\varepsilon}$ to $\mathbb{L}_{\left\{\Lambda^{\varepsilon}\right\}}\left(L_{\infty, 1}, C_{\infty}\right)$; then the above inequality, together with $E \mapsto \mathrm{h}_{(E)}$ being Lipschitz to $\mathbb{L}_{\left\{\Lambda^{\varepsilon}\right\}}\left(C_{\infty}, C_{\infty}\right)$, implies that $E \mapsto \mathrm{h}_{(E)}$ is Lipschitz from $B_{\varepsilon}$ to $\mathbb{L}_{\left\{\Lambda^{\varepsilon}\right\}}\left(L_{\infty, 1}, C_{\infty}\right)$; use now prop. 6 .

\footnotetext{
${ }^{25}$ Dependence of $h$ on $E$ will be omitted when no ambiguity arises as a result.
} 
Hence $\mathrm{h}$ has the same properties as $\mathrm{k}_{k}^{k}$, so the first equation too can be given the above operator interpretation; so $\mathrm{h}-\mathbf{1}$ is the (left- and right-) inverse of $\mathrm{k}_{k}^{k}-\mathbf{1}$ in $\mathbb{L}\left(L_{\infty, 1}, L_{\infty, 1}\right)$ too - and the same argument applies (going through Fubini again $)$ in all $\mathbb{L}\left(L_{\infty, 1}^{\lambda}, L_{\infty, 1}^{\lambda}\right), \lambda \in \Lambda^{\varepsilon}$. Finally, for $f \in L_{p, q}^{\lambda}$, thus $=e^{-\lambda t} g_{t}$, $g \in L_{p, q}$, we majorise $\mathrm{k}_{k}^{k}$ by its upper bound: $\left\|\mathrm{k}_{k}^{k}(f)\right\|_{p, q}^{\lambda}=\left\|e^{\lambda x} \mathrm{k}_{k}^{k}\left(e^{-\lambda t} g_{t}\right)\right\|_{p, q} \leq$ $\left\|\mathrm{k}_{k}^{k}\right\|_{\Lambda^{\varepsilon}}^{L C}\left\|\frac{e^{\lambda z}}{\psi_{\varepsilon}(z)} \star g\right\|_{p, q} \leq\left\|\mathrm{k}_{k}^{k}\right\|_{\Lambda^{\varepsilon}}^{L C}\left\|\frac{e^{\lambda z}}{\psi_{\varepsilon}(z)}\right\|_{1}\|f\|_{p, q}^{\lambda}$, by [20, thm. 2]. By shrinking again a bit $\Lambda^{\varepsilon},\left\|\frac{e^{\lambda z}}{\psi_{\varepsilon}(z)}\right\|_{1}<\infty$, and hence $\mathrm{k}_{k}^{k}$ (and thus $\mathrm{h}$ too) belong to $\mathbb{L}\left(L_{p, q}^{\lambda}, L_{p, q}^{\lambda}\right.$ ) $\forall \lambda \in \Lambda^{\varepsilon}, \forall p, q$. Since $\mathrm{k}_{k}^{k}-\mathbf{1}$ and $\mathrm{h}-\mathbf{1}$ are inverses on the encompassing space $L_{\infty, 1}^{\lambda}$, it follows they are still inverses in all $\mathbb{L}\left(L_{p, q}^{\lambda}, L_{p, q}^{\lambda}\right)$, and similarly for the $C_{p}^{\lambda}$.

Remark 11. This shows our results on invertibility are quite sharp: either the spectrum of $\mathrm{k}$ at a BGE contains 1, i.e., for some $\omega, e^{\mathrm{i} \omega t}$, and hence the 2-dimensional space of linear combinations of $\cos (\omega t)$ and $\sin (\omega t)$, solves the linearisation of the fixed point problem - so, $\frac{\partial F}{\partial k}$ is not even injective on $C_{\infty}$, the basic space for $k$ - , or there exists a full neighbourhood of 0 , both for $E$ and for $\lambda$, where $\frac{\partial F}{\partial k}$ is invertible, with the same (cf. App. B) inverse $\mathrm{h}-\mathbf{1}$, in all the above operator spaces.

\subsection{Kernel representations of the derivatives of $\varpi$.}

Identifying discontinuous part of each kernel reduces the numerical problem to that of computing their continuous parts (chiefly that of lemma 4, the others are just a matter of integration) ${ }^{26}$ which are everywhere well-defined, thus turning the problem into a "well-posed problem". Else kernels would just be maps to equivalence classes of measurable functions: quite unrealistic to compute.

Lemma 5. The derivative of the $C_{\infty}$-components of $\varpi$ w.r.t. $E$ is a kernel operator, and for $i$ it is a kernel operator of the difference $\varpi_{y}^{\prime}-\varpi_{c}^{\prime}$ plus $x \mapsto \int \delta E_{x, s} d s$.

Each kernel is a function of $(x, t, t-s)$ and is a sum of a continuous function of $x$ and

- for $k: \xi_{R}(x-t)$.

- for $y$ and $\mathfrak{r}$ : the same term, multiplied resp. by $\alpha y_{x} / k_{x}$ and $\alpha(1-\alpha) y_{x} / k_{x}^{2}$.

- for $c: \mathrm{k}_{E}^{c}(x ; t, t-s)$ (cf. lemma 1.ic).

- for $i$ : $\alpha \frac{y_{x}}{k_{x}} \xi(x-t)-\mathrm{k}_{E}^{c}(x ; t, t-s)$.

Those kernels compose properly ${ }^{27}$ with the partial derivatives in lemma 1. Similarly, the kernels of lemmas 1 .viii and 4 compose properly to that of $\varpi_{k}^{\prime}$.

Proof. All the kernels from lemma 1 used in this proof will be evaluated at the solution $\varpi(E)$ corresponding to $E$ from a ball $B$ where lemma 3 applies.

Since $\varpi_{k}^{\prime}=-\left(\frac{\partial F}{\partial k}\right)^{-1} \frac{\partial F}{\partial E}$, by lemmas 1 .iii and 4

$$
\varpi_{k}^{\prime}=\delta E \mapsto\left[x \curvearrowright-\iiint \mathrm{k}_{E}^{k}(z ; t, t-s) \delta E_{t, s} d s d t\left(\mu_{E}^{k}(x)\right)(d z)\right]
$$

By lemma 1.x, Fubini's theorem applies here, i.e., with $\varrho_{E}^{k}$ from lemma 1.viii, $\varpi_{k}^{\prime}=$ $\delta E \mapsto\left[x \curvearrowright-\int \varrho_{E}^{k}\left(\mu^{k}(x) ; t, t-s\right) \delta E_{t, s} d s d t\right]$. Recall, by definition of $\mu^{k}$ in lemma 4.iii, $\mu^{k}(x, d z)=\mathrm{h}(x, z) d z-\epsilon_{x}(z)$, where $\mathrm{h}(x, z) d z=\mathrm{h}_{c}(x, z) d z-\alpha \frac{y_{t}}{k_{t}} \xi_{R}(x-z) d z$ is non-atomic by lemma 4. So, $\varrho_{E}^{k}\left(\mu^{k}(x) ; \cdot, \cdot\right)=\varrho_{E}^{k}(\mathrm{~h}(x, z) d z ; \cdot, \cdot)-\varrho_{E}^{k}\left(\epsilon_{x}(z) ; \cdot, \cdot\right)$.

\footnotetext{
${ }^{26}$ Re-expressing equation 2 in lemma 4 .ii (which defines $\mathrm{h}$ ) with the continuous part $S$ of $\mathrm{h}$ gives $S(x, z)=\int S(x, t) \mathrm{k}(t, z) d t$ plus a known continuous function; solve that equation numerically. The $\|\cdot\|_{\Lambda^{\varepsilon}}^{L C}$ bound of lemma $4 . \mathrm{i}$ should prove very useful for the numerical analysis of this (e.g., local density of a grid); however to investigate appropriate truncations it seems "stability" results for the kernels themselves would be needed. That is beyond our scope here.

${ }^{27}$ I.e., if $\delta X=\sum_{i} A_{i} \delta Y_{i}$ is such an equation, where $X$ and the $Y_{i}$ are equilibrium variables in some spaces $S$ and $S_{i}$, and $A_{i} \in \mathbb{L}\left(S_{i}, S\right)$ is represented by a kernel $a_{i}$ at $\varpi(E)$, then, if $y_{i}$ is the kernel representing $\varpi_{Y_{i}}^{\prime}(E)$ at $\varpi(E), a_{i}$ and $y_{i}$ compose properly as kernels, the composite kernel applied to any $\delta E \in L_{\infty, 1}$ yields the same as $A_{i} \circ \varpi_{Y_{i}}^{\prime}(E)$ (i.e., an appropriate form of Fubini is applicable), and those composite kernels sum exactly to the kernel specified for $\varpi_{X}^{\prime}(E)$.
} 
Then, by lemma 1.ix, $\varrho_{E}^{k}(\mathrm{~h}(x, z) d z ; \cdot, \cdot)$ is continuous in $x$. By definition of $\varrho_{E}^{k}$ and the decomposition $\mathrm{k}_{E}^{k}=\mathrm{k}_{E}^{k d}-\mathrm{k}_{E}^{k c}$ (in lemma 1), $\varrho_{E}^{k}\left(\epsilon_{x} ; \cdot, \cdot\right)=\varrho_{E}^{k d}\left(\epsilon_{x} ; \cdot, \cdot\right)-$ $\varrho_{E}^{k c}\left(\epsilon_{x} ; \cdot, \cdot\right)$. For the kernel of $\varpi_{k}^{\prime}$ it is left to show that the second term is a continuous function of $x$, while the first one is $\xi_{R}(x-t)$ and thus has a discontinuity at $x=t . \varrho_{E}^{k c}\left(\epsilon_{x} ; t, u\right)=\int \mathrm{k}_{E}^{k c}(z, t, u) \epsilon_{x}(z) d z=\mathrm{k}_{E}^{k c}(x, t, u)$, continuous in $x$ by lemma 1.vi. $\varrho_{E}^{k d}\left(\epsilon_{x} ; t\right)=\int \xi_{R}(z-t) \epsilon_{x}(z) d z=\xi_{R}(x-t)$.

Since $\varpi_{y}^{\prime}(\delta E)=\frac{\partial y}{\partial k} \circ \varpi_{k}^{\prime}(\delta E)$ and $\varpi_{\mathfrak{r}}^{\prime}=\frac{\partial \mathfrak{r}}{\partial k} \circ \varpi_{k}^{\prime}$, the result for $y, \mathfrak{r}$ follows from lemma 1.ia-ib.

For $c$ : $\varpi_{c}^{\prime}(\delta E)=\frac{\partial c}{\partial E} \delta E+\frac{\partial c}{\partial k} \circ \varpi_{k}^{\prime}(\delta E)$, hence the kernel of the derivative is, by the above and lemma1.ic, $\mathrm{k}_{E}^{c}(x, t, t-s)-\varrho_{E}^{k}\left(\mathrm{k}_{k}^{c}(x, z) \mu_{E}^{k}(z), t, t-s\right)$. The first term is discontinuous at $x=t+s$ (cf. notation 5.1), while the second term is continuous in $x$ by lemma 1.ic.

For $i$ : the result then follows by lemma 1.ie, indeed, $\varpi_{i}^{\prime}(\delta E)(x)=$

$$
\int\left(\varrho_{E}^{k}\left(\left(\mathrm{k}_{k}^{c}(x, z)-\alpha \frac{y_{x}}{k_{x}}\right) \mu_{E}^{k}(z), t, t-s\right)-\mathrm{k}_{E}^{c}(x ; t, t-s)\right) \delta E_{t, s} d s d t+\int \delta E_{x, s} d s
$$

so the last term is the ("direct shock") part of the derivative that is given by a unit mass at $x, \epsilon_{x}$, (thus not a classical function of $x$, hence not part of the kernel) while the first one is a kernel which (by the above) has a discontinuous part $\mathrm{k}_{E}^{c}$ that has a jump at $x=t+s$.

To verify the proper composition of kernels we need to show that the composition of $\partial \Upsilon / \partial k$ with the derivative $\varpi_{k}^{\prime}$ plus $\partial \Upsilon / \partial E$ equals $\varpi_{k}^{\prime}$. Indeed, using lemma 1.iiiiv,

$$
(\delta \Upsilon(\delta k, \delta E))(x)=\iint \mathrm{k}_{E}^{k}(x ; t, t-s) \delta E_{t, s} d t d s+\int \mathrm{k}_{k}^{k}(x ; u) \delta k_{u} d u
$$

and so the composed kernel is

$$
\mathrm{k}_{E}^{k}(x ; t, t-s)-\int \mathrm{k}_{k}^{k}(x ; u) \int \mathrm{k}_{E}^{k}(z ; t, t-s)\left(h(u, z)-\epsilon_{u}(z)\right)(d z) d u
$$

which, by Fubini, equals

$\mathrm{k}_{E}^{k}(x ; t, t-s)-\int \mathrm{k}_{E}^{k}(z ; t, t-s)\left(\int \mathrm{k}_{k}^{k}(x ; u) h(u, z) d u\right) d z+\iint \mathrm{k}_{E}^{k}(z ; t, t-s) \mathrm{k}_{k}^{k}(x ; u) \epsilon_{u}(z)(d z) d u$

and then, by lemma 4.ii, it equals

$\mathrm{k}_{E}^{k}(x ; t, t-s)-\int \mathrm{k}_{E}^{k}(z ; t, t-s)\left(\mathrm{k}_{k}^{k}(x ; z)+h(x, z)\right) d z+\int \mathrm{k}_{E}^{k}(u ; t, t-s) \mathrm{k}_{k}^{k}(x ; u) d u=$

$\mathrm{k}_{E}^{k}(x ; t, t-s)-\int \mathrm{k}_{E}^{k}(z ; t, t-s) h(x, z) d z=\int \mathrm{k}_{E}^{k}(z ; t, t-s)\left(\epsilon_{x}(z)-h(x, z)\right) d z$

which is the kernel of $\varpi_{k}^{\prime}$ as a function of $(x, t, t-s)$.

\section{Main Results}

10.1. Regularity and stability of solutions. Here we finally present our main results, applying lemma 3 that is based on the implicit function theorem to a solution of the equilibrium system and derive the smoothness properties of each solution $\varpi$ as a function of the exogenous $E$ defined in a neighbourhood $B$ of zero $(E=0)$. For most of what follows we write $\varpi(E)$ to indicate this dependence. Recall that $\varpi(E)$ itself is an array of functions $(k, c, y, \mathfrak{r}, i)$, where the first four are continuous and bounded, and hence will be referred to as $C_{\infty}$ components, while $i \in L_{\infty, 1}$; when referring to a particular component, its name will be used as a subscript, and since all these functions are defined on $\mathbb{R}$, the value returned by the function will be referred to in a usual fashion, so that, e.g., $\left(\varpi_{k}(E)\right)(x)$ will denote that value of capital $(k)$ at time $x$ of a solution (in the neighbourhood 
of BGE $\varpi(0))$ corresponding to endowment path $E$. Likewise, the derivative, $\varpi^{\prime}$, too, depends on $E$, as in lemma 3. Derivative itself, recall, maps $\delta E$, an element of $L_{\infty, 1}$, to the variation in the solution, i.e., a profile $(\delta k, \delta c, \delta y, \delta \mathfrak{r}, \delta i)$. So, e.g., $\left(\left(\varpi_{k}^{\prime}(E)\right)(\delta E)\right)(x)$ denotes the change (variation) in capital $\delta k$ at time $x$ [from the initial level $\left.\left(\varpi_{k}(E)\right)(x)\right]$ as a result of a perturbation $\delta E$ of endowments from their initial level $E$.

Recall, again that the set $\mathcal{G} \subseteq \wp$ is, as in prop. 3 , a generic subset of parameters where $\partial F / \partial k$ is invertible. Also, $\psi_{\varepsilon}$ is as defined in notation 9.1 and so is $\|\cdot\|_{\Lambda^{\varepsilon}}^{L C}$. $\lambda_{+}$and $\lambda_{-}$are from cor. 6 .

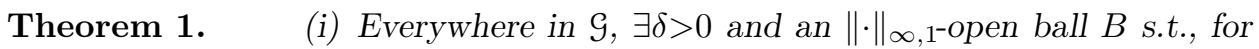
any $B G E \varpi(0), \forall E \in B$, there is a unique solution $\varpi(E) \in P$ with $\| \varpi_{k}(E)-$ $\varpi_{k}(0) \| \leq \delta$, and s.t. $\varpi$ is Fréchet- $C^{1}$ on $B$.

(ii) Further, $\forall \varepsilon>0$ there is an $\|\cdot\|_{\infty, 1}$-open ball $B_{\varepsilon} \subseteq B$ s.t., $\forall B G E$ there is a compact interval $\Lambda^{\varepsilon} \subseteq \Lambda$ with $\max \left\{\min \left\{0, \lambda_{-}+\varepsilon\right\}, \frac{-1}{\varepsilon}\right\}$ and $\left(\lambda_{+}-\varepsilon\right)^{+}$in its interior, s.t., on $B_{\varepsilon}$ :

(a) $E \mapsto \varpi_{q}^{\prime}(E)$ is Lipschitz from $\|\cdot\|_{\infty, 1}$ to $\|\cdot\|_{\Lambda^{\varepsilon}}^{L C}$ for any component $q \in\{k, y, c, \mathfrak{r}, i\}$ of $\varpi$.

(b) $\varpi$ is differentiable in the strong sense: for any component $q \in\{k, y, c, \mathfrak{r}, i\}$ of $\varpi$ there is $L \in \mathbb{R}: \forall x$,

$\left|\left(\varpi_{q}(E+\delta E)\right)(x)-\left(\varpi_{q}(E)\right)(x)-\left(\left(\varpi_{q}^{\prime}(E)\right)(\delta E)\right)(x)\right| \leq L\|\delta E\|_{\infty, 1}\left[\frac{1}{\psi_{\varepsilon}} \star \int|\delta E(\cdot, s)| d s\right](x)$

(c) $\exists L: \forall \lambda \in \Lambda^{\varepsilon}, \forall p,\left\|\varpi_{q}\left(E_{1}\right)-\varpi_{q}\left(E_{2}\right)\right\|_{p}^{\lambda} \leq L\left\|E_{1}-E_{2}\right\|_{p}^{\lambda}$ for any component $q \in\{k, y, c, \mathfrak{r}, i\}$ of $\varpi$.

With $\mathrm{k}_{E}^{q}$ the kernel of the derivative of a $C_{\infty}$-component $q \in\{k, y, c, \mathfrak{r}\}$ of $\varpi, K^{q} \stackrel{\text { def }}{=} \sup _{B_{\varepsilon}}\left\|\mathrm{k}_{E}^{q}\right\|_{\Lambda^{\varepsilon}}^{L C}$ and $\delta E=E_{1}-E_{2}, \forall x$,

$\left|\left(\varpi_{q}\left(E_{1}\right)\right)(x)-\left(\varpi_{q}\left(E_{2}\right)\right)(x)\right| \leq K^{q}\left[\frac{1}{\psi_{\varepsilon}} \star \int|\delta E(\cdot, s)| d s\right](x)$

and with $\mathrm{k}_{E}^{i}$ the kernel (part) of the derivative $\varpi_{i}^{\prime},{ }^{28}$

$$
\left|\left(\varpi_{i}\left(E_{1}\right)\right)(x)-\left(\varpi_{i}\left(E_{2}\right)\right)(x)-\int \delta E_{x, s} d s\right| \leq K^{i}\left[\frac{1}{\psi_{\varepsilon}} \star \int|\delta E(\cdot, s)| d s\right](x)
$$

(d) For $\lambda \in \Lambda^{\varepsilon}, \delta E \mapsto \varpi_{q}(E+\delta E)-\varpi_{q}(E)$ is sequentially continuous from $\left(L_{1}^{\lambda}, \sigma\left(L_{1}^{\lambda}, L_{\infty}^{-\lambda}\right)\right)$ to $C_{1}^{\lambda}$ for any $C_{\infty}$-component $q \in\{k, y, c, \mathfrak{r}, i\}$ of $\varpi$ and to $\left(L_{1}^{\lambda}, \sigma\left(L_{1}^{\lambda}, L_{\infty}^{-\lambda}\right)\right)$ for the $i$-th component.

Remark 12 . While points iia-iib express the regularity aspect (i.e., $C^{1}$ ) in a sharper way, points iic-iid express a very strong form of stability, or "no hysteresis": that the effects of a perturbation decay exponentially at rates $\lambda_{+}$and $\lambda_{-}$at $+\infty$ and $-\infty$.

But, it is point iia which is the basic one, since point iib follows from it and points iic and iid follow from iib: regularity and stability are a single theorem!

Remark 13. (iid) is a "weak" analogue of lemma 3.ii. Observe that, even with $\lambda=0$ and at $E=0$, next result cannot extend to $L_{p, 1}$ instead of $L_{1}$ : shifting $\delta E$ yields then a sequence converging weakly to 0 , while the corresponding equilibria are also obtained by shifting, hence cannot converge to 0 in $C_{p}$.

Proof. (i): By lemma 3 and [20, prop.3.v].

(iia): For the $C_{\infty}$-components use lemma 5 and prop. 6 . The result for $i$ follows too, since the non-kernel part is constant in $E$ and the kernel represents a linear operator from $L_{\infty, 1}\left(\mathbb{R}^{2}\right)$ to $C_{\infty}$ (being the difference of the kernels of $\varpi_{y}^{\prime}$ and $\varpi_{c}^{\prime}$ ).

\footnotetext{
${ }^{28}$ By lemma 5 this kernel is that of $\varpi_{y}^{\prime}-\varpi_{c}^{\prime}$.
} 
(iib): Denote by $\mathrm{k}_{E}$ the kernel of the derivative, $\varpi_{q}(E)$, of a $C_{\infty}$-component $q$ of the solution ${ }^{29}$ evaluated at $E$, each such kernel is well-defined by lemma 5 . Let

$$
g_{z} \stackrel{\text { def }}{=}\left(\varpi_{q}(E+z \delta E)\right)(x)-\left(\varpi_{q}(E)\right)(x)-z \iint \mathrm{k}_{E}(x ; t, t-s) \delta E_{t, s} d s d t
$$

then, since by point i, $\varpi$ is Fréchet and hence Gateaux differentiable,

$$
g_{z}^{\prime}=\iint\left[\mathrm{k}_{E+z \delta E}(x ; t, t-s)-\mathrm{k}_{E}(x ; t, t-s)\right] \delta E_{t, s} d s d t
$$

Hence, $\left|g_{z}^{\prime}-g_{0}^{\prime}\right| \leq \iint\left|\mathrm{k}_{E+z \delta E}(x ; t, s)-\mathrm{k}_{E}(x ; t, s) \| \delta E_{t, s}\right| d s d t$. By point iia and prop. $6, \exists L$ s.t. $\forall x,\left|\mathrm{k}_{E+\delta E}(x ; t, u)-\mathrm{k}_{E}(x ; t, u)\right| \leq L\|\delta E\|_{\infty, 1} \frac{1}{\psi_{\varepsilon}(x-t)}$ a.e. Hence $\left|g_{z}^{\prime}\right| \leq L z\|\delta E\|_{\infty, 1}\left[\frac{1}{\psi_{\varepsilon}} \star \int|\delta E(\cdot, s)| d s\right](x)$. Since $\frac{1}{\psi_{\varepsilon}} \in L_{1, \infty}$, convolution with it $\epsilon$ $\mathbb{L}\left(L_{\infty, 1}, C_{\infty}\right)$ by $[20$, thm. 1.xiii], hence the bound is finite for any finite $z$. Integrate now over $z \in[0,1]$. (iia).

The result for the $i$-th component then follows, exactly as in the proof of point

(iic): The first claim is from lemma 3.ii. For the pointwise bound, replace $\mathrm{k}_{E}^{q}$ by its upper bound and $\delta E$ by $|\delta E|$ in the derivative, and integrate on the segment joining $E_{1}$ and $E_{2}$.

(iid): $B_{\varepsilon}$ is weakly closed in $L_{1}^{\lambda}$ by lemma 21 . For the $C_{\infty}$-components, (iic) implies, by the " $\lambda$-transposition" of $[20$, cor. 6$]$ by lemma 6 (in app. A), that, with $E_{2}=E, E_{1}=E+\delta E$, and $\delta E$ in a weakly compact set in $L_{1}^{\lambda}$, the $\mid \varpi(E+\delta E)-$ $\varpi(E) \mid$ are majorised by a compact family in $C_{1}^{\lambda}$. Conclude by [20, lemma 1] and lemma 21. The result for $i$ follows by lemma 5 .

10.2. From solutions to equilibria. To extend the results of the main theorem to equilibria as well, one has to assure that the solution emerging as a result of a perturbation of policy $(\delta E)$ also satisfies the interiority constraints, i.e., individual consumption is positive and the two inequalities $0<i_{t}<y_{t}$ hold. Hence, in addition to the assumptions of the previous theorem, one has to assure that in the neighbourhood where the theorem holds first, the life-time transfers are not too negative for almost all individuals, so that $\mathscr{N}_{x}$, the normalised individual income, is positive, ${ }^{30}$ and, second, the aggregate transfer is uniformly bounded, and hence the last two inequalities hold.

Theorem 2. $\exists \delta_{1}>0$ such that, $\forall B G E, \mathscr{N}$ of the equilibrium in the neighbourhood of a given $B G E,\left\{E \in B \mid\right.$ ess sup $\left._{x} \int E_{x+s, s}^{-} d s \leq \delta_{1}\right\}$, is uniformly bounded away from 0 on that neighbourhood.

$\delta$ can be chosen in thm. 1 and $\exists \delta_{0}>0$, s.t., on the set where $\left\|\int E ._{s} d s\right\|_{\infty} \leq \delta_{0}$ and

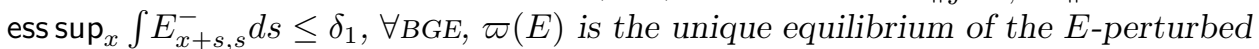
economy s.t. $\left\|\varpi_{k}(E)-\varpi_{k}(0)\right\| \leq \delta$ and $\left\|\varpi_{c}(E)-\varpi_{c}(0)\right\| \leq \delta$.

Proof. By prop. 1.iii-v, for sufficiently small $\delta_{1}, \mathscr{N}_{x} \geq \varepsilon$, since $\varpi_{\mathfrak{r}}$ is bounded and $\varpi_{k}$, thus $\varpi_{y}$, bounded away from 0 .

To show that $\varpi(E)$ is an equilibrium, we need, by prop. 1 , that $\mathscr{N} \geq 0$, and that, if not in the basic model, $0<i_{t}<y_{t}$ a.e., since inf $k_{t}>0$ by cor. 7 .

$\varpi$ is continuous by thm. 1 , so the theorem remains true for any smaller $\delta$, by adjusting $B$. By taking $\delta$ sufficiently small $\varpi_{y}(E)-\varpi_{y}(0)$ and $\varpi_{c}(E)-\varpi_{c}(0)$ will be uniformly small, hence $i_{t}=y_{t}+\int E_{t, s} d s-c_{t}$ implies that $i-i^{*}$ will be uniformly arbitrarily small if $\left\|\int E{ }_{., s} d s\right\|_{\infty} \leq \delta_{0}$ for sufficiently small $\delta_{0}$; in particular, $0<i_{t}<y_{t}$ will hold: $\varpi(E)$ is an equilibrium.

\footnotetext{
${ }^{29}$ So that $q \in\{k, y, c, \mathfrak{r}\}$.

${ }^{30}$ Since it has the same sign as consumption of an individual born at $x$ (cf. sect. 3.2).
} 
Remains the uniqueness part. Since the equality $i_{t}=y_{t}+\int E_{t, x} d s-c_{t}$ holds for any equilibrium, the same argument as above shows that, for $\delta$ and $\delta_{0}$ sufficiently small the inequality $0<i_{t}<y_{t}$ will hold; prop. 1 implies then that the whole equation system must hold, so the equilibrium must be $\varpi(E)$, by the uniqueness statement in thm. 1. And for the basic model, the result follows from $k_{t}>0$ (cor. 7).

Remark 14. Just for $p=\infty$, since $\Lambda^{\varepsilon}$ can be taken as a compact interval approximating $\Lambda$ as close as desired from inside (so with 0 interior), the theorem implies a very strong form of stability (i.e., uniform convergence to the status-quo), towards both $-\infty$ and $+\infty$, "at any exponential rate in $\Lambda$ ".

\subsection{Technical remarks (on the choice of the underlying spaces).}

Remark 15. The usual (strong) definition of a function being $C^{k}$ on a set is that the function has a $C^{k}$ extension to some open set containing the given set. Using this is the only way to distinguish the adequate topologies (for describing continuity, derivatives, etc.) from the region where the implicit function exists. Our results are exactly of this sort, the 'solutions' of thm. 1 being the required extension.

This approach does use the fact that demand extends naturally and smoothly for $M<0$. Nothing is specific to this example there: we mentioned in [18] that homogeneity of utility w.r.t. to consumption goods was essential for balanced growth, and this seems indeed a pure implication of homogeneity. E.g., if labour does not enter the utility function, as here, homogeneity implies directly that demand is positively homogeneous of degree 1 in $M$. The extension used is the obvious one, making demand homogeneous of degree 1 in $M$ : we did not use any accidental feature there of our specification of the model.

Remark 16. Conditions for regularity of the BGE's w.r.t. variations in the parameters are trivial: it suffices that when restricting all functions in $\Upsilon$ in prop. 1 to be constants, at each BGE $\frac{d \tilde{k}}{d k} \neq 1$, i.e., equivalently $\widehat{\mathbb{k}}_{k}^{k}(0) \neq 1$. In particular, on the generic set $\mathcal{G}$, regularity w.r.t. variations in the parameters also holds.

Corollary 8. On the set of E's described in thm. 2, the $k, y, \mathfrak{r}$ components of $\varpi$ have values in a $\tau\left(C_{\infty}, M\right)$ compact set. If the ess sup condition in thm. 2 were strengthened to ess $\sup _{x} \int\left|E_{x+s, s}\right| d s \leq \delta_{0}$, the whole map $\varpi$ in thm. 1 would become compact-valued on this domain.

Proof. As seen above, in that case the $\varpi_{i}$ are bounded in $L_{\infty}$, so, with the weak* topology, they live in a compact metric space. On that space, first $k$, then $y, \mathfrak{r}$, then the others are, as in the proof of lemma 21, continuous functions of $i$. If further the $\int\left|E_{x+s, s}\right| d s$ are bounded in $L_{\infty}$, this implies that the $\mathscr{N}_{1, x}$ are so, and one can then do a similar argument for the remaining components using in addition the relative compactness of the $\mathscr{N}_{1, x}$.

Remark 17 . The second case in the corollary suggests to reinterpret $E_{x+s, s} d s$ as a measure $\mu_{x}(d s)$ on $[0,1]$ (or a single measure on $\mathbb{R} \times[0,1]$ ), with the weak topology of the dual of $L_{1}^{C([0,1])}(\mathbb{R})$. The second constraint identifies a ball in this space, compact and metrisable in this duality; and the first constraint defines then a closed subset, inducing the $\sigma\left(L_{\infty}, L_{1}\right)$ topology on the $\int E_{., s} d s$. The equilibrium equations obviously still make sense with such endowments, since they imply prices are even $C^{1}$, and this would make $\varpi$ a continuous function on a compact metric space.

\section{Conclusions}

We have demonstrated that "comparative statics" around a BGE in an overlapping generations model in continuous time with respect to a fully anticipated transfer is feasible. Perhaps, the most time-consuming part of the work is to calculate the 
derivative of the equilibrium map, but we provided an array of auxiliary statement in the appendix for some shortcuts both in calculation and in showing the required smoothness $\left(S^{1}\right)$ of the derivative. Although tedious, such work has a high payoff: the implicit function theorem assures not only the existence of the one-to-one map between the "policy parameter" (transfer of endowments) and the resulting equilibrium in the neighbourhood of the chosen BGE, but also the Lipschitz property of its derivative, hence assuring the first order approximation of that map given by the derivative is "of high quality." Moreover, the same theorem also yields exponential convergence of the equilibrium variables back to the status-quo (initial BGE) and we provide a way to calculate (using numerical methods if needed) the maximal speed of such convergence in both directions in time.

\section{Appendix A. Auxiliary statements: Concepts And tools}

Lemma 6. Denote by $\nu^{\star}$ the convolution with $\nu$.

(i) For $\nu \in M^{\lambda}$, next diagram commutes:

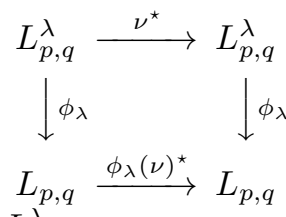

(ii) For $\mu, \nu \in M^{\lambda}, \mu^{\star} \circ \nu^{\star}=(\mu \star \nu)^{\star}$ on all $L_{p, q}^{\lambda}$.

(iii) $\|\nu \star f\|_{p, q}^{\lambda} \leq\|\nu\|^{\lambda}\|f\|_{p, q}^{\lambda}$.

(iv) $M^{\lambda}$ injects with algebra homomorphisms of norm 1 into the operator algebras on all $L_{p, q}^{\lambda}$ and $C_{p}^{\lambda}$; and $\phi_{\lambda}: M^{\lambda} \rightarrow M$ is an algebra-isomorphism.

(v) $\|f g\|_{p, q}^{\lambda_{1}+\lambda_{2}} \leq\|f\|_{p_{1}, q_{1}}^{\lambda_{1}}\|g\|_{p_{2}, q_{2}}^{\lambda_{2}}$ for $\frac{1}{p_{1}}+\frac{1}{p_{2}}=\frac{1}{p}, \frac{1}{q_{1}}+\frac{1}{q_{2}}=\frac{1}{q}$

Proof. i is clear. ii follows by juxtaposing 2 such diagrams, convolution being well defined on $M^{\lambda}$ (def. 7). iii and $\mathrm{v}$ follow from the case $\lambda=0$ ([20, theorems 2.ii and 1.xiii]), and iv from ii and iii.

\section{A.1. Kernels.}

Lemma 7. For a topological space $T$, and a $\sigma$-finite measure $\nu$ on the Baire $\sigma$-field of $T$, let $\mathrm{k}: T \rightarrow L_{1}(\nu)$ be bounded, continuous, and have values in a separable subspace of $L_{1}(\nu)$. If $\mathbf{1}-\mathrm{k}$ is invertible in $\mathbb{L}\left(C_{\infty}, C_{\infty}\right)$, the inverse is of the same form $\mathbf{1}-\mathrm{h}$, where $\mathrm{h}$ is also bounded and continuous to $L_{1}(\nu) . \mathrm{h}$ and $\mathrm{k}$ can be chosen jointly (Baire-)measurable; then $\mathrm{h}$ is the unique solution of $\mathrm{h}(x, \cdot)=$ $-\mathrm{k}(x, \cdot)+\int \mathrm{h}(t, \cdot) \mathrm{k}(x, t) \nu(d t) \nu$-a.e. s.t. $x \mapsto \mathrm{h}(x, t) \nu(d t)$ is weak ${ }^{*}$-continuous and bounded. The same holds for the equation $\mathrm{h}(x, \cdot)=-\mathrm{k}(x, \cdot)+\int \mathrm{k}(t, \cdot) \mathrm{h}(x, t) \nu(d t)$.

Proof. Replace $\nu$ by an equivalent finite measure. The inverse must be a bounded weak*-continuous map $\mu$ from $X$ to $C_{\infty} *$. Since k has values in a separable subspace of $L_{1}(\nu)$, [9] implies that it has, for some separable sub- $\sigma$-algebra of the Baire $\sigma$-field, a jointly measurable version $\mathrm{k}(x, t) \nu(d t)$. Then $(\mathbf{1}-\mathrm{k}) \mu=\mathbf{1}$ becomes $\int \mu(t, \cdot)\left(\epsilon_{x}(t)-\mathrm{k}(x, t) \nu(d t)\right)=\epsilon_{x}$, i.e., $\mu(x, \cdot)=\epsilon_{x}+\int \mu(t, \cdot) \mathrm{k}(x, t) \nu(d t)$. Let $\xi=$ $\int \mu(t, \cdot) \nu(d t)$, viewed as a measure on the Stone space $X$ of $T$ (the space of characters of $\left.C_{\infty}\right)$ : the measures in the right hand integral are for all $x$ absolutely continuous w.r.t. $\xi$, so one can write $\mu(x, d z)=\epsilon_{x}-\mathrm{l}(x, z) \xi(d z)$, where again $\mathrm{l}(x, \cdot)$ is normcontinuous and bounded to $L_{1}(\xi), \mu$ being bounded. The separability asumption on $\mathrm{k}$ implies that, for some sequence $x_{n}$, the $\mathrm{k}\left(x_{n}, \cdot\right)$ are dense in the set of all $\mathrm{k}(x, \cdot)$ in $L_{1}(\nu)$. Thus the $\mathrm{l}(x, z) \xi(d z)$ lie again in a separable subspace of $L_{1}(\xi)$; since such a subspace is generated by a separable sub- $\sigma$-algebra, one can choose by [9] 1 to be jointly Baire measurable. In terms of 1 , the equation for $\mu$ becomes $\mathrm{l}(x, z) \xi(d z)=-\mathrm{k}(x, z) \nu(d z)+\left(\int \mathrm{k}(x, t) \mathrm{l}(t, z) \nu(d t)\right) \xi(d z)$, where now $\nu$ is also viewed as a measure on $X$, and $\mathrm{k}$ and $\mathrm{l}$ are extended in an arbitrary Baire-measurable 
way to $X \times X$, but still the equation holds only for $x \in T$. There is a use of Fubini's theorem for the last term; since $\mathrm{k}$ and $\mathrm{l}$ are jointly measurable, it just needs $\int|\mathrm{k}(x, t)||\mathrm{l}(t, z)| \nu(d t) \xi(d z)<\infty$. Since $\mathrm{l}$ is bounded to $L_{1}(\xi)$ and $\mathrm{k}$ to $L_{1}(\nu)$, this holds. This equation for 1 is an equality of 2 measures: so if one kills the singular part of $\xi$ w.r.t. $\nu$, the equality is still satisfied; thus, let $\xi^{a}$ denote the non-singular part of $\xi$ w.r.t. $\nu$, then $\mu^{a}(x, d z) \stackrel{\text { def }}{=} \epsilon_{x}-1(x, z) \xi^{a}(d z)$ is also a (right)inverse of $\mathbf{1}-\mathrm{k}$, since the continuity and boundedness of 1 implies that $x \mapsto \mu^{a}(x, \cdot)$ is weakly-continuous and bounded. By the uniqueness of the inverse, one has thus $\mu=\mu^{a}$. With $f$ a density of $\xi^{a}$ w.r.t. $\nu$ and $\mathrm{h}(x, z)=\mathrm{l}(x, z) f(z)$ one has thus $\mathrm{h}$ jointly measurable, $\mu(x, d z)=\epsilon_{x}-\mathrm{h}(x, z) \nu(d z), x \mapsto \mathrm{h}(x, \cdot)$ is bounded and continuous to $L_{1}(\nu)$, and $\mathrm{h}$ is the solution of the equation $\forall x, \mathrm{~h}(x, z)=-\mathrm{k}(x, z)+$ $\int \mathrm{k}(x, t) \mathrm{h}(t, z) \nu(d t) \nu(d z)$-a.e.

For the uniqueness statement: $x \mapsto \mathrm{h}(x, t) \nu(d t)$ weak*-continuous and bounded ensures $\mu \in \mathbb{L}\left(C_{\infty}, C_{\infty}\right)$, and the equation expresses that $\mu$ is a right inverse of $1-\mathrm{k}$. Doing the same with the left inverse yields the other equation.

Remark 18. This lemma generalises to kernels a substantial part of Wiener's theorem (used previously in prop. 2, and which is a core point of our whole argument): that inverses tend to have similar properties (lie "in the same space") as the inversee.

Proposition 4. Let $A$ be a kernel operator. Then $\exists \mathrm{k}: \mathbb{R}^{n} \times \mathbb{R}^{m} \rightarrow \mathbb{R}$ Borel s.t. $\forall f \in L_{\infty}, \forall s \in \mathbb{R},[A(f)](s)=\int \mathrm{k}(s, t) f(t) d t$. Further $\sup _{s} \int|\mathrm{k}(s, t)| d t=\|A\|<\infty$, and $A$ is continuous under the Mackey topologies $\tau\left(L_{\infty}, L_{1}\right)$ and $\tau\left(C_{\infty}, M\right)$.

Proof. Let $k_{s}: L_{\infty} \rightarrow \mathbb{R}: f \mapsto[A(f)](s): k_{s} \in L_{\infty}^{*}$, and the pointwise convergence condition ensures then $k_{s} \in L_{1}$. Doob's [9] classical martingale argument yields then a jointly borel version $\mathrm{k}(s, t)$. The first point in the 'further' clause is obvious; it allows to use Fubini's theorem to obtain $\int[A(f)](s) \varsigma(d s)=\int \mathrm{k}(\varsigma, t) f(t) d t \forall f \in$ $L_{\infty}, \forall \varsigma \in M$ where $\mathrm{k}(\varsigma, t)=\int \mathrm{k}(s, t) \varsigma(d s)$. This implies that $A^{t}: \varsigma \mapsto \mathrm{k}(\varsigma, \cdot)$ is $\sigma\left(M, C_{\infty}\right)-\sigma\left(L_{1}, L_{\infty}\right)$ continuous, and thus $A$, by duality, Mackey continuous.

Corollary 9. For a kernel $\mathrm{k}$ on $\mathbb{R}^{2}$, let $\|\mathrm{k}\|^{*}=\| y \mapsto$ ess $\sup _{x, t} \mathbb{1}_{|t-x+y| \leq \frac{1}{2}}\left|\mathrm{k}_{x, t}\right| \|_{1}$. $\exists K: \forall p, \mathrm{k}, f,\left\|\int \mathrm{k}_{x, t} f_{t} d t\right\|_{p, \infty} \leq K\|\mathrm{k}\|^{*}\|f\|_{p, 1}$.

Proof. Let $h_{y}=\operatorname{essup}_{t}\left|\mathrm{k}_{t+y, t}\right|$ and apply [20, prop. 2], using [0,1] for $B$ on $\mathbb{R}$.

Lemma 8. Let $h: \mathbb{R} \rightarrow\left(L_{\infty}, \sigma\left(L_{\infty}, L_{1}\right)\right): x \mapsto h_{x}$ be bounded and continuous, with sup-norm $\|h\|_{\infty}$, and s.t. $h_{x}(s)$ is null for $|s|>a$. For $f \in L_{p, 1}^{\lambda}, F: x \mapsto$ $\int f(x+s) h_{x}(s) d s \in C_{p}^{\lambda}$, and $\|F\|_{p}^{\lambda} \leq K_{\lambda}\|h\|_{\infty}\|f\|_{p, 1}^{\lambda}$, where $K_{\lambda}=K(1+2 a) e^{|\lambda| a}$.

Remark 19. In particular, $\mathrm{k}_{x, t}=h_{x}(x-t)$ is a kernel operator.

Proof. $a>0$, else the result is trivial. For a jointly borel version of $h[9]$, let $\mathrm{k}_{x, t}=e^{\lambda(x-t)} h_{x}(t-x)$. Cor. 9, applied to $\phi_{\lambda}(f)$ and $\phi_{\lambda}(F)$, implies $\|F\|_{p}^{\lambda} \leq$ $K\|\mathrm{k}\|^{*}\|f\|_{p, 1}^{\lambda}$, with $\|\mathrm{k}\|^{*}=\left\|y \mapsto \operatorname{ess} \sup _{x, s} e^{\lambda s} \mathbb{1}_{|s-y| \leq \frac{1}{2}}\left|h_{x}(-s)\right|\right\|_{1}, \leq\|h\|_{\infty} \| y \mapsto$ $\sup _{s} \mathbb{1}_{|s-y| \leq \frac{1}{2},|s| \leq a} e^{\lambda s} \|_{1}$. The latter norm equals $e^{|\lambda| a}+\frac{1}{\lambda}\left[e^{\lambda a}-e^{-\lambda a}\right]$; use then $\frac{e^{x}-e^{-x}}{x} \leq 1+e^{|x|}$.

Remains thus to prove continuity of $F .|F(x+\varepsilon)-F(x)| \leq\|h\|_{\infty} \int_{x-a}^{x+a} \mid f(t+$ $\varepsilon)-f(t)|d t+| \int_{-a}^{a} f(x+s)\left(h_{x+\varepsilon}(s)-h_{x}(s)\right) d s \mid$. Since $f \in L_{p, 1}^{\lambda}$ implies $f$ is locally integrable, the first term converges to 0 by the continuity property in [20, thm. 2.iii] (taking $\mu$ as a unit mass at $-\varepsilon$ ), and the second by definition of $\sigma\left(L_{\infty}, L_{1}\right)$, hence the continuity.

Corollary 10. For $\xi_{R}(x)=\mathbb{1}_{x \geq 0} e^{-R x}(R>0)$ and $g \in L_{\infty}(\mathbb{R} \times \Omega)$, let $h_{x}(\omega)=$ $\left(\xi_{R} \star g(\cdot, \omega)\right)(x)$. Then $x \mapsto h_{x}$ is Lipschitz to $L_{\infty}(\Omega)$.

Proof. Since $z \mapsto \mathbb{S}_{z} \xi_{R}: \mathbb{R} \rightarrow L_{1}$ is Lipschitz, use [20, prop. 2]. 


\section{A.2. Elementary operations.}

Lemma 9. The product from $L_{\infty} \times L_{\infty, 1}$ to $L_{\infty, 1}$ is $S^{1}$.

Proof. By lemma 6.v, $\|f g\|_{p, 1}^{\lambda} \leq\|f\|_{p, \infty}^{\lambda}\|g\|_{\infty, 1}$ and $\|f g\|_{p, 1}^{\lambda} \leq\|f\|_{\infty}\|g\|_{p, 1}^{\lambda}$. Thus in [20, prop. 4], using there $\varphi$ for the product map, the $\left\|\varphi^{i}\right\|_{\alpha}$ are all $\leq 1$, so $\|\varphi\|_{S}$ is too.

Remark 20. $L_{\infty}$, as a Banach algebra under multiplication, turns all $L_{p, q}^{\lambda}$ spaces and their finite intersections into $L_{\infty}$-modules, and the operator norm on $L_{\infty}$ induced by any of those equals the $L_{\infty}$-norm. The same holds for $C_{\infty}$ and the $C_{p}^{\lambda}$ spaces. In particular, with $A=\{(\lambda, p, q) \mid 1 \leq p, q \leq \infty\}$, and any $\mathcal{A}$, the Banach families $L_{\infty}$ with the $\|\cdot\|_{p, q}^{\lambda}$ and $C_{\infty}$ with $\|\cdot\|_{p}^{\bar{\lambda}}$ satisfy as Banach-algebras all conditions of [20, cor. 8].

Lemma 10. The product on $L_{\infty}(\mathbb{R} \times[0,1])$ and on $C_{\infty}(\mathbb{R} \times[0,1])$ is $S^{1}$.

Proof. Same as previous proof (or: [20, cor.8] and rem. 20).

Lemma 11. The tensor product (product of measure spaces, and functions thereon) $\operatorname{maps} L_{p, q}^{\lambda_{1}}\left(\mathbb{R}^{n}\right) \times L_{p, q}^{\lambda_{2}}\left(\mathbb{R}^{k}\right) \rightarrow L_{p, q}^{\left(\lambda_{1}, \lambda_{2}\right)}\left(\mathbb{R}^{n} \times \mathbb{R}^{k}\right)$, with $\|f g\|_{p, q}^{\left(\lambda_{1}, \lambda_{2}\right)} \sim\|f\|_{p, q}^{\lambda_{1}}\|g\|_{p, q}^{\lambda_{2}}$.

Proof. Reduce to $\lambda_{i}=0$ by the definition and use Fubini's theorem.

Corollary 11. $f \mapsto f g: L_{\infty, 1}\left(\mathbb{R}^{n}\right) \rightarrow L_{\infty, 1}\left(\mathbb{R}^{n} \times \mathbb{R}^{k}\right)$ is $S^{1}$ iff $g \in L_{1}$.

Proof. By lemma 11 and [20, thm. 1.viii].

Remark 21. This will be used for the product $y \varphi$ in the proof of lemma 1.ic. It shows that there is no hope to make this map jointly $S^{1}$ in any reasonable sense, so some special treatment or new inventiveness will be needed when dealing also with regularity (differentiability) issues w.r.t. parameters like $\varphi$. Possibly treating them just as Banach spaces rather than families (like $E$ ); but then we'll need more about the interaction between those 2 structures.

Lemma 12. For $n>0$ and $\mu \in M, f \mapsto \int f(x, s) \mu(d s): C_{\infty}\left(\mathbb{R}^{n+1}\right) \rightarrow C_{\infty}\left(\mathbb{R}^{n}\right)$ is $S^{1}$.

Proof. $\left\|\int f(x, s) \mu(d s)\right\|_{p, \infty}^{\lambda} \leq\|\mu\| \sup _{s}\left\|x \mapsto e^{\lambda x_{1}} f(x, s)\right\|_{p, \infty} \leq\|\mu\|\|f\|_{p, \infty}^{\lambda}$, by the trivial inequality $\|x \mapsto g(x, s)\|_{p, \infty} \leq\|g\|_{p, \infty} \forall s$.

Lemma 13. For $n>0, h: L_{\infty, 1}\left(\mathbb{R}^{n+1}\right) \rightarrow L_{\infty, 1}\left(\mathbb{R}^{n}\right): f \mapsto \int_{0}^{1} f(x, s) d s$ is $S^{1}$.

Proof. Let $B$ be an open ball in $\mathbb{R}^{n}$, and $B^{\prime}=B \times[-1,1] \subseteq \mathbb{R}^{n+1}$. For the induced norms [[20, thm. 1.ii] — note the constants are independent of $p, q]$, if $p<\infty$, $\left(\|h(f)\|_{p, 1}^{\lambda}\right)^{p}=\int\left(\int_{x+B} e^{\lambda y_{1}}\left|\int_{0}^{1} f(y, s) d s\right| d y\right)^{p} d x \leq \int\left(\int_{x+B} \int_{0}^{1} e^{\lambda y_{1}}|f(y, s)| d s d y\right)^{p} d x$ $\leq \int\left(\int_{(x, s)+B^{\prime}} e^{\lambda z_{1}}|f(z)| d z\right)^{p} d x \forall s \in[0,1]$, thus $\leq \int\left(\int_{(x, s)+B^{\prime}} e^{\lambda z_{1}}|f(z)| d z\right)^{p} d x d s$ $=\int\left(\int_{y+B^{\prime}} e^{\lambda z_{1}}|f(z)| d z\right)^{p} d y=\left(\|f\|_{p, 1}^{\lambda}\right)^{p}$. Also $\|h(f)\|_{\infty, 1}^{\lambda}=\sup _{x \in \mathbb{R}^{n}} \int_{x+B} e^{\lambda y_{1}}$ $\left|\int_{0}^{1} f(y, s) d s\right| d y \leq \sup _{x \in \mathbb{R}^{n+1}} \int_{x+B^{\prime}} e^{\lambda z_{1}}|f(z)| d z=\|f\|_{\infty, 1}^{\lambda}$. Thus $\left\|h^{i}\right\|_{\alpha} \leq 1$ (notation of [20, prop. 4]) and so $\|h\|_{S} \leq 1$; hence the result by [20, prop. 4].

Corollary 12. $h$ of lemma 13 is in $\mathbb{L}\left(L_{p, 1}^{\lambda}\left(\mathbb{R}^{n+1}\right), L_{p, 1}^{\lambda}\left(\mathbb{R}^{n}\right)\right)$.

Lemma 14. $T:(f, g) \mapsto T_{f, g}: x \curvearrowright \int_{-a}^{a} f_{x, s} g_{x+s, s} d s$ is $S^{1}$ from $L_{\infty} \times L_{\infty, 1}$ to $L_{\infty, 1}$.

Proof. Since $g \mapsto\left[(x, s) \curvearrowright \mathbb{1}_{|s| \leq|a|} g_{x+s, s}\right]$ is clearly $S^{1}$ from $L_{\infty, 1}$ to itself $\left(\|\cdot\|_{p, 1}^{\lambda}\right.$ changes by at most a factor $e^{|\lambda a|}$ ), the result follows from lemmas 9 , then 13 .

Lemma 15. $T:(f, g) \mapsto \int_{-a}^{a} f_{x, s} g_{x+s} d s$ is $S^{1}$ from $L_{\infty} \times L_{\infty, 1}$ to $L_{\infty}$.

Let $L C_{\infty}=\left\{f: \mathbb{R} \rightarrow\left(L_{\infty}, \sigma\left(L_{\infty}, L_{1}\right)\right) \mid x \mapsto f_{x}\right.$ is bounded and continuous $\}$. It maps to a closed subspace of $L_{\infty}\left(\mathbb{R}^{2}\right)$. Then $T$ is $S^{1}$ from $L C_{\infty} \times L_{\infty, 1}$ to $C_{\infty}$. 


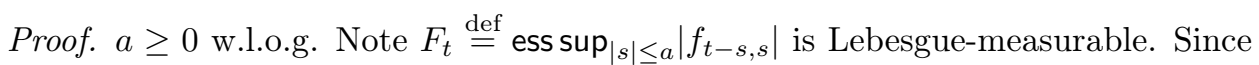
$|T| \leq \int_{x-a}^{x+a} F_{t}\left|g_{t}\right| d t,\|T\|_{p, \infty}^{\lambda} \leq K_{\lambda}\|F g\|_{p, 1}^{\lambda}$ by lemma 8 , and the last norm is bounded by both $\|F\|_{\infty}\|g\|_{p, 1}^{\lambda}$ and $\|F\|_{p, \infty}^{\lambda}\|g\|_{\infty, 1}$ using lemma 6.v (in app. A). Since, for any measurable set $B$, ess sup $\mathbb{1}_{B} F=\operatorname{ess} \sup \mathbb{1}_{x+s \in B,|s| \leq a}\left|f_{x, s}\right|,\|F\|_{p, \infty}^{\lambda}=\|f\|_{p, \infty}^{\lambda}$, up to a factor $e^{|\lambda| a}$, when defining the latter norm by the measurable set $|x+s| \leq 1,|s| \leq a$ ([20, thm.1.ii)]. Thus $\forall S \in \mathcal{A} \exists K_{S}$ s.t., for $(\lambda, p) \in S,\|T\|_{p, \infty}^{\lambda}$ is bounded by both $K_{S}\|f\|_{\infty}\|g\|_{p, 1}^{\lambda}$ and $K_{S}\|f\|_{p, \infty}^{\lambda}\|g\|_{\infty, 1}$ : by [20, prop. 4], the bilinear map $(f, g) \mapsto T$ has finite \|\|$\cdot\|\|_{S}$-norm for all $S \in \mathcal{A}$, hence is $S^{1}$.

The rest follows now from lemma 8 , using a jointly Borel version of $f$, cf. [9].

\section{A.3. Composition with $C^{1}$ functions on $L_{\infty}$.}

Lemma 16. For $O$ open in $\mathbb{R}^{n}$, let $\tilde{O}$ be the interior in $L_{\infty}^{\mathbb{R}^{n}}$ of $\left\{g \in L_{\infty}^{\mathbb{R}^{n}} \mid g_{t} \in O \forall t\right\}$. If $f: O \rightarrow \mathbb{R}$ is differentiable with locally Lipschitz derivative, then $\tilde{f}: \tilde{O} \rightarrow$ $L_{\infty}: g \mapsto f \circ g$ is $S^{1}$, with $D_{g}: L_{\infty}^{\mathbb{R}^{n}} \rightarrow L_{\infty}: \delta g \mapsto\left(t \curvearrowright\left\langle\nabla f_{g_{t}}, \delta g_{t}\right\rangle\right)$ as derivative at $g$. The same holds when replacing $L_{\infty}$ by $C_{\infty}$.

Proof. Since $g \in \tilde{O},\left(\frac{\partial f}{\partial x^{i}}\right)_{g_{t}} \in L_{\infty}$, thus $D_{g} \in \mathbb{L}\left(L_{\infty}^{\mathbb{R}^{n}}, L_{\infty}\right)$. With $K$ the (compact) closure of $\left\{g_{t}\right\}$, modulo null sets, apply [20, prop. 3.i] to $f$ (which is $S^{1}$ ): $\exists \varepsilon>0, \exists L$ : $\|\delta g\|_{\infty} \leq \varepsilon \Rightarrow\left\|f\left(g_{t}+\delta g_{t}\right)-f\left(g_{t}\right)-\left\langle\nabla f_{g_{t}}, \delta g_{t}\right\rangle\right\|_{\infty} \leq L\left(\|\delta g\|_{\infty}\right)^{2}$, thus differentiability.

Remains the local Lipschitz aspect of $g \mapsto D_{g}$. Use the arbitrary given norm on $\mathbb{R}^{n}$ to define the sup-norm of $\delta g$ or $g \in L_{\infty}^{\mathbb{R}^{n}}$, and its dual to define the norm of gradients on $\mathbb{R}^{n}$; by rem. 20 , the operator norm on $D_{g}$ is then the sup-norm, also on $C_{\infty} \cap C_{p}^{\lambda}$. By [20, lemma 4], $\exists \delta>0, L$ s.t. $x \mapsto \nabla_{x} f$ has Lipschitz constant $L$ on the $\delta$-neighbourhood of $K$; so, for $\left\|g_{i}-g\right\|_{\infty}<\delta,\left\|D_{g_{1}}-D_{g_{2}}\right\|_{\infty} \leq L\left\|g_{1}-g_{2}\right\|_{\infty}$.

Clearly $\tilde{f}(g)$ and $D_{g}(\delta g)$ are continuous if $g$ and $\delta g$ are so.

Remark 22. Another instructive way to proceed would be to use [20, cor. 8] (cf. rem. 20), to get immediately the result for $f$ polynomial (by [20, prop. 5]); remains then to develop the tools to handle the approximation argument.

Lemma 17. For $\vartheta \in L_{\infty}$ with bounded support, let $k_{x, t}=\vartheta_{t-x} \exp \int_{x}^{t} g_{y} d y$. Then $g \mapsto k$ is $S^{1}$ from $L_{\infty, 1}$ to $L_{\infty}$.

Proof. Let Supp $\vartheta \subseteq[-a, a] . \quad G: g \curvearrowright\left[(x, t) \mapsto G_{g}(x, t) \stackrel{\text { def }}{=} \mathbb{1}_{|x-t| \leq a} \int_{x}^{t} g_{y} d y\right]$ is linear, and by lemma 8, letting $F_{x}=\int_{x-a}^{x+a}\left|g_{y}\right| d y,\|F\|_{p, \infty}^{\lambda} \leq K_{\lambda}\|g\|_{p, 1}^{\lambda}$; so, since $\left|G_{g}(x, t)\right| \leq \mathbb{1}_{|x-t| \leq a} F_{x},\left\|G_{g}\right\|_{p, \infty}^{\lambda} \leq K_{\lambda}\|g\|_{p, 1}^{\lambda}$. $K_{\lambda}$ being bounded on each $S \in \mathcal{A}$, $G \in \mathbb{L}^{\Lambda}\left(L_{\infty, 1}, L_{\infty}\right)$, so $G$ is $S^{1}$ from $L_{\infty, 1}$ to $L_{\infty}$. Conclude by [20, prop. 5], since $G \mapsto k$ is $S^{1}$ from $L_{\infty}$ to itself, by lemma 16 and then rem. 20 and [20, cor. 8].

Lemma 18. With $k$ of lemma 17, let $T:(g, h) \mapsto T_{g, h}: x \curvearrowright \int k_{x, t} h_{t, t-x} d t$, and $T_{g, h}^{\prime}:(\delta g, \delta h) \curvearrowright\left[x \mapsto T_{g, \delta h}(x)+\int \psi_{x, t} \delta g_{t} d t\right]$ with $\psi_{x, t}=\int_{t}^{(t-x) \cdot(+\infty)} k_{x, y} h_{y, y-x} d y$. Then $T$ is $S^{1}$ from $L_{\infty, 1} \times L_{\infty, 1}$ to $L_{\infty, 1}$, with derivative $T^{\prime}$.

Proof. The $S^{1}$ aspect follows by [20, prop. 5] and lemmas 17 and 14 .

As to $T^{\prime}$, the partial w.r.t. $h$ is obvious; the other is a priori $\int k_{x, t} h_{t, t-x} \int_{x}^{t} \delta g_{y} d y d t$, going to the stated form is then an elementary change of order of integration.

Lemma 19. Assume $h_{t, s}=z_{t}$ in lemma 18. Then $T$ is $S^{1}$ from $L_{\infty, 1} \times L_{\infty, 1}$ to $C_{\infty}$. Proof. Follows by [20, prop. 5] and lemmas 17 and 15 .

Lemma 20. For $\lambda<R, i \mapsto k$ is $\left(L_{p, 1}^{\lambda}, C_{p}^{\lambda}\right)$-continuous, with norm $<K\left(1+\frac{1}{R-\lambda}\right)$. It is sequentially continuous from $L_{1}^{\lambda}$ with the weak topology to $C_{1}^{\lambda}$. 
Proof. Let $g_{t}=e^{\lambda t} i_{t}, G_{t}=e^{\lambda t} k_{t}$ : then $G_{t}=e^{-(R-\lambda) t} \int_{-\infty}^{t} e^{(R-\lambda) s} g_{s} d s ;$ since $\lambda<R$, suffices to consider the case $\lambda=0$. Further, continuity of $k$ is obvious. [20, prop. 2.i] implies the bound since $k=i \star\left[e^{-R t} \mathbb{1}_{t \geq 0}\right]$ and $\left\|e^{-R t} \mathbb{1}_{t \geq 0}\right\|_{1, \infty}=1+\frac{1}{R}$. Similarly [20, cor.6] implies the second statement.

A.4. Simpler norms for $\mathbb{L}_{\Lambda}\left(C_{\infty}, C_{\infty}\right)$ and $\mathbb{L}_{\Lambda}\left(L_{\infty, 1}, C_{\infty}\right)$.

Proposition 5. For $Q \in \mathbb{L}\left(C_{\infty}, C_{\infty}\right)$, represented by a kernel $\mu(x, d t)$, and $J$ a compact interval, $\|Q\|_{J}$ is stronger than $\|\mu\|_{J}^{C C}$.

If $J^{\prime}$ is interior to $J,\|\mu\|_{J}^{C C}$ is stronger than $\|Q\|_{J^{\prime}}$.

Proof. Replacing the $\|\cdot\|_{p, q}^{\lambda}$ in the definition of the $\|\cdot\|_{J}$ operator-norm by their definition in terms of $\|\cdot\|_{p, q}$ (notation 4.2 and 9.1) yields $\|Q\|_{J}=\sup _{\lambda \in J} \sup _{p \in[1, \infty]} \sup _{\|f\|_{p, \infty} \leq 1} N$, with $N=\left\|\int e^{\lambda(x-t)} f_{t} \mu(x, d t)\right\|_{p, \infty}$. Fix $p=\infty$; the norm in $N$ is $\geq \sup _{x}$, since $Q \in \mathbb{L}\left(C_{\infty}, C_{\infty}\right):\|Q\|_{J} \geq \sup _{x} \sup _{\lambda \in J} \sup _{\|f\|_{\infty} \leq 1} \int e^{\lambda(x-t)} f_{t} \mu(x, d t),=\sup _{x} \sup _{\lambda \in J}$ $\int e^{\lambda(x-t)}|\mu(x, d t)|$. Restrict now $\lambda$ to $\min J$ and $\max J$, and add.

Assume $\left(\psi_{J} \star|\mu(x, \cdot)|\right)(x) \leq K \forall x$, and $J^{\prime} \pm \varepsilon \subseteq J$. Let $F(z)=1 / \psi_{-\varepsilon, \varepsilon}(z)$ : for $\lambda \in J^{\prime}, e^{\lambda z} / \psi_{J}(z) \leq F(z)$. Thus, with $H(x, d t)=\psi_{J}(x-t)|\mu(x, d t)|,\|H(x, \cdot)\|_{1} \leq$ $K$ and $N \leq\left\|\int F(x-t)\left|f_{t}\right| H(x, d t)\right\|_{p, \infty} \leq K\left\|\sup _{t} F(x-t)\left|f_{t}\right|\right\|_{p, \infty}$. Since $F \in L_{1, \infty}$, the result follows by [20, cor. 2].

Proposition 6. For a kernel $\mathrm{k}(x ; t, u)$ representing some $Q \in \mathbb{L}\left(L_{\infty, 1}\left(\mathbb{R}^{2}\right), C_{\infty}\right)$, $\|Q\|_{J}$ is stronger than $\|\mathrm{k}\|_{J}^{L C}$.

If $J^{\prime}$ is interior to $J$, and $\mathrm{k}=0$ for $|s|>a,\|\mathrm{k}\|_{J}^{L C}$ is stronger than $\|Q\|_{J^{\prime}}$.

Proof. $\|Q\|_{J}=\sup _{\lambda \in J, p \in[1, \infty]} \sup _{\|f\|_{p, 1} \leq 1}\left\|\iint e^{\lambda(x-t)} \mathrm{k}(x ; t, t-s) f_{t, s} d s d t\right\|_{p, \infty}$, by notation 4.2 and 9.1 .

Therefore $\|Q\|_{J} \geq\|\mathrm{k}\|^{\prime} \stackrel{\text { def }}{=} \sup _{\lambda \in J} \sup _{\|f\|_{\infty, 1} \leq 1}\left\|\iint e^{\lambda(x-t)} \mathrm{k}(x ; t, t-s) f_{t, s} d s d t\right\|_{\infty, \infty}$, $=\sup _{x} \sup _{\lambda \in J} \sup _{\|f\|_{\infty, 1} \leq 1} \iint e^{\lambda(x-t)} \mathrm{k}(x ; t, t-s) f_{t, s} d s d t$. By the converse in [20, thm. 1.xiii] this is equivalent to $\sup _{x} \sup _{\lambda \in J}\left\|e^{\lambda(x-t)} \mathrm{k}(x ; t, u)\right\|_{1, \infty}$. Since, for $\lambda \in J$, $e^{\lambda z} \leq \psi_{J}(z),{ }^{31}$ the triangle inequality implies $\|\mathrm{k}\|^{\prime}$ is still equivalent to $\sup _{x} \| \psi_{J}(x-$ $t) \mathrm{k}(x ; t, u) \|_{1, \infty}$, which is stronger than $\|\mathrm{k}\|_{J}^{L C}$ by $[20$, thm. 1.viii].

With $g(z)=1 / \psi_{J}(z),\left|\mathrm{k}_{x ; t, u}\right| \leq\|\mathrm{k}\|_{J}^{L C} g(x-t) .\left\|\iint e^{\lambda(x-t)} \mathrm{k}(x ; t, t-s) f_{t, s} d s d t\right\|_{p, \infty}$ clearly increases when replacing $\mathrm{k}$ and $f$ by their absolute values; so, by the above formula, $\|\mathrm{k}\|_{J^{\prime}} \leq\|\mathrm{k}\|_{J}^{L C} \sup _{\lambda \in J^{\prime}, p \in[1, \infty]} \sup _{\|f\|_{p, 1} \leq 1}\left\|\phi_{\lambda}(g) \star h(f)\right\|_{p, \infty}$, where $h: L_{\infty, 1}\left(\mathbb{R}^{2}\right) \rightarrow L_{\infty, 1}(\mathbb{R}): f \mapsto \int_{-a}^{a} f(x, s) d s ;$ and by notation $4.2, \phi_{\lambda}(g)=[t \mapsto$ $\left.e^{\lambda t} g(t)\right]$. Since $\|f\|_{p, 1} \leq 1$, by cor. $12\|h(f)\|_{p, 1}<\infty$, so by [20, prop. 2] $\exists K \in \mathbb{R}$ s.t. $\left\|\phi_{\lambda}(g) \star h(f)\right\|_{p, \infty} \leq K\left\|\phi_{\lambda}(g)\right\|_{1, \infty}$. Thus $\|\mathrm{k}\|_{J^{\prime}} \leq K\|\mathrm{k}\|_{J}^{L C} \sup _{\lambda \in J^{\prime}}\|g\|_{1, \infty}^{\lambda}$, and the latter sup is clearly finite.

\section{A.5. The weak topologies.}

Lemma 21. Closed balls $B$ in $L_{\infty, 1}$ are " $\lambda$-closed": for $f_{\alpha} \in B, f_{\alpha}-f_{\alpha_{0}} \rightarrow g$ $\sigma\left(L_{\infty, 1}^{\lambda}, L_{1, \infty}^{-\lambda}\right)$ implies $f_{\alpha} \rightarrow g+f_{\alpha_{0}}$ in $\left(B, \sigma\left(L_{\infty, 1}, L_{1, \infty}\right)\right)$. $\varpi$ is sequentially continuous on $\left(B, \sigma\left(L_{\infty, 1}, L_{1, \infty}\right)\right)$ - for the $C_{\infty}$-components, to the topology of uniform convergence on compact sets, and for the others, to $\sigma\left(L_{\infty, 1}, L_{1, \infty}\right)$.

Proof. $\lambda$-closedness follows from $B=\left\{f \mid\|h\|_{\infty} \leq 1 \Rightarrow\left\langle f, h \mathbb{1}_{[x-1, x]}\right\rangle \leq R_{B} \forall x\right\}$.

For the sequential continuity, let $E_{n} \rightarrow E_{\infty}$ in $\left(B, \sigma\left(L_{\infty, 1}, L_{1, \infty}\right)\right)$. By cor. 7 , the $C_{\infty}$-components of $\varpi\left(E_{n}\right)$ are uniformly bounded; extract a $\sigma\left(L_{\infty}, L_{1}\right)$-convergent subsequence. Then since $i=E+y-c, i_{n} \rightarrow i_{\infty} \sigma\left(L_{\infty, 1}, L_{1, \infty}\right)$. So, since clearly $\mathbb{1}_{s \leq t} e^{R s} \in L_{1, \infty}, k_{n} \rightarrow k_{\infty}$ pointwise by prop. 1.x. By the local uniform integrability of the $i_{n}$, the variation of the $k_{n}$ over short intervals tends locally uniformly to 0 ; in particular, the $k_{n}$ are equicontinuous, hence $k_{n} \rightarrow k_{\infty}$ uniformly on compact

\footnotetext{
${ }^{31}$ Recall, by notation $9.1, \psi_{\lambda, \lambda^{\prime}}(z)=e^{\lambda z}+e^{\lambda^{\prime} z}$, and for a compact interval $J, \psi_{J}=\psi_{\min J \max J}$.
} 
sets. The same holds thus by prop. $1 . \mathrm{i}$ and ii also for $y_{n} \rightarrow y_{\infty}$ and $f_{n} \rightarrow f_{\infty}$, since $k$ is uniformly bounded away from 0 (cor.7). The same holds then for $c$ too, going through the successive points of prop. $1\left(\mathscr{N}, \mathscr{N}_{1}, \mathscr{B}\right.$ and $\mathscr{B}_{1}$ converge $\sigma\left(L_{\infty, 1}, L_{1, \infty}\right)$, the others uniformly on compact sets): either use an argument like for $k$, or that a weakly convergent sequence in $L_{1}$ converges uniformly on Mackeycompact subsets of $L_{\infty}$. Finally, the above also shows that the limit variables $E_{\infty}, i_{\infty}, k_{\infty}, y_{\infty} \ldots$ solve the equation system in prop. 1 . Thus, by the uniqueness part in lemma $3,\left(i_{\infty}, k_{\infty}, y_{\infty}, \ldots\right)=\varpi\left(E_{\infty}\right)$. Hence the limit is independent of the chosen subsequence, so the convergence holds for the original sequence.

\section{Appendix B. Discontinuity, in terms of $\lambda$, of the InVERsion}

To see better the nature of the difficulty, why we obtain speeds of convergence only for $\lambda \in \Lambda$, and not $\forall \lambda \leq R$, consider the kernels $\varphi_{\alpha}(x)=\operatorname{sign}(\alpha) \mathbb{1}_{\alpha x>0} e^{-\alpha x}$ for $\alpha \neq 0$. They are a simplified version of $\mathbb{k}_{k}^{k}$, with its main qualitative features. We get $\widehat{\varphi}_{\alpha}=y \mapsto \frac{1}{\alpha-\mathrm{i} y}$, hence $\widehat{\varphi}_{\alpha}-\widehat{\varphi}_{\beta}+(\alpha-\beta) \widehat{\varphi}_{\alpha} \widehat{\varphi}_{\beta}=0$, so $\varphi_{\alpha}-\varphi_{\beta}+(\alpha-\beta) \varphi_{\alpha} \star \varphi_{\beta}=0$, and thus, with $f=A \varphi_{\alpha}$, denoting by $g_{0}$ the solution in $L_{1}$ of the convolution equation $f+g=f \star g$ (i.e., $\mathbb{1}-g_{0}$ is the inverse in the Wiener algebra of $\mathbb{1}-f, \mathbb{1}$ denoting the identity): $g_{0}=-A \varphi_{\alpha-A}$ for $A \neq \alpha$.

Observe that $\phi_{\lambda}\left(\varphi_{\alpha}\right) \in L_{1}$ - i.e., $\varphi_{\alpha} \in L_{1}^{\lambda}-$ iff $\alpha(\alpha-\lambda)>0$, and then $\phi_{\lambda}\left(\varphi_{\alpha}\right)=\varphi_{\alpha-\lambda}$ and if $\alpha(\alpha-\lambda) \leq 0$ then $\phi_{\lambda}\left(\varphi_{-\alpha}\right)=\varphi_{\alpha-\lambda}$. Thus, by the inversion formula applied to $f=A \varphi_{\lambda} \in L_{1}^{\bar{\lambda}}$, for such $\lambda$ the solution in $L_{1}^{\lambda}$ of our convolution equation equals $g_{0}$ if $(A-\alpha)(A-\alpha+\lambda)>0$, and for $(A-\alpha)(A-\alpha+\lambda)<0$ equals $g_{*}=-A \varphi_{A-\alpha}$, so $g_{0}-g_{*}=A \operatorname{sign}(A-\alpha) e^{(A-\alpha) x}$. In particular for each $\lambda$ at most 1 of $g_{0}$ and $g_{*}$ belongs to $L_{1}^{\lambda}$, since an exponential belongs to no such space. Note there exists $\lambda$ such that $f$ and $g_{*} \in L_{1}^{\lambda}$ (i.e., $\alpha(\alpha-\lambda)>0$ and $(A-\alpha)(A-\alpha+\lambda)<0$ ) iff $\alpha A>0$ and $A \neq \alpha$. Assume this henceforth.

We have thus 2 solutions $g_{0}$ and $g_{*}$ of our convolution equation, so the difference, hence $e^{(A-\alpha) x}$, belongs to $\operatorname{Ker}(\mathbb{1}-f)$ in $L_{\infty}^{\alpha-A}$. And $\mathbb{1}-f$ is trivially invertible on $L_{1}^{\lambda} \cap L_{\infty}$ if the inverse on $L_{1}^{\lambda}$ equals $g_{0}$, i.e., for $(A-\alpha)(A-\alpha+\lambda)>0$. Else, if $(A-\alpha)(A-\alpha+\lambda) \leq 0$, note that $e^{(A-\alpha) x}$ is a continuous linear functional on $L_{1}^{\lambda} \cap L_{\infty}$, and denote by $K$ its kernel: $K=\left\{h \in L_{1}^{\lambda} \cap L_{\infty} \mid \int h(x) e^{(A-\alpha) x} d x=0\right\}$. Clearly $K$ is the set where $g_{0}$ and $g_{*}$ coincide, hence the inverses of $\mathbb{1}-f, \mathbb{1}-g_{0}$ on $L_{\infty}$ and $\mathbb{1}-g_{*}$ on $L_{1}^{\lambda}$, coincide on $h \in L_{1}^{\lambda} \cap L_{\infty}$ iff $h \in K$; thus the image of $L_{1}^{\lambda} \cap L_{\infty}$ by $\mathbb{1}-f$ equals $K$. And for $h \notin K,\left(\mathbb{1}-g_{0}\right)(h) \notin L_{1}^{\lambda}$ and $\left(\mathbb{1}-g_{*}\right)(h) \notin L_{\infty}$.

\section{Appendix C. Zeros of a multivariate anAlytic FunCtion}

Lemma 22. Let $f: O \rightarrow \mathbb{R}$ be analytic and non-null, where $O$ is open and connected in $\mathbb{R}^{n}$. Then the set of zeros of $f$ is closed and negligible.

Remark 23. The same statement holds with the same proof replacing $\mathbb{R}$ by $\mathbb{C}$.

Remark 24. The conclusion can obviously be strengthened to 0 measure for any measure whose conditionals on any factor given the other factors are non-atomic.

Proof. For $n=0$ the statement is trivial. Proceeding by induction, let the statement hold for $n-1$. Assume first $O$ is a product of two open connected sets $X \times Y$, $X \in \mathbb{R}^{n-1}, Y \in \mathbb{R}$. By assumption there is a point $\left(x^{0}, y^{0}\right) \in X \times Y$ at which $f$ is non-null. Then by the induction hypothesis the set of zeros in $X$ of $f\left(x, y^{0}\right)$, $Z_{y_{0}} \subset X$, is closed and has measure zero. For any fixed $x \in X \backslash Z_{y_{0}}, f(x, y)$ is an analytic function defined on $Y$, non-zero (at $y_{0}$ ), thus the set of its zeros, $Z_{x}$, is discrete. The set of zeros of $f$ on $X \times Y$ then is a union of $\left\{\left(x, y_{0}\right): x \in Z_{y_{0}}\right\}$ and $\left\{(x, y): y \in Z_{x}\right\}$, both of measure zero. For general $O$, cover then $O$ with countably many products of the form $X \times Y$; since we know the set of zeros is closed in $O$, it suffices to show that its intersection with each of those product sets has measure 
0 . This follows from our previous argument provided $f$ does not vanish identically on any of those product sets. But if it did, connexity of $O$ would imply by analytic continuation that $f$ vanishes everywhere on $O$.

\section{REFERENCES}

[1] Allais, M. (1947): Economie et intérêt. Imprimerie Nationale, Paris.

[2] Burke, Jonathan L. (1990): "A benchmark for comparative dynamics and determinacy in overlapping-generations economies". Journal of Economic Theory, vol. 52(2), pp. 268-303, ISSN 0022-0531, doi:10.1016/0022-0531(90)90034-H. URL http://www.sciencedirect.com/ science/article/pii/002205319090034H.

[3] CAss, D. and M. E. YAARI (1967): "Individual Saving, Aggregate Capital Accumulation, and Efficient Growth". In Essays on the Theory of Optimal Economic Growth, edited by Shell, K., pp. 233-268, MIT Press, Cambridge (MA).

[4] Chichilnisky, Graciela and Ylaging ZhOU (1998): "Smooth infinite economies". Journal of Mathematical Economics, vol. 29, pp. 27-42.

[5] D'Albis, H. and E. Augeraud-VÉron (2009): "Competitive growth in a life-cycle model: existence and dynamics". International Economic Review, vol. 50(2), pp. 459-484, ISSN 1468-2354.

[6] D'Albis, Hippolyte and Emmanuelle Augeraud-VÉron (2007): "Balanced cycles in an OLG model with a continuum of finitely-lived individuals". Economic Theory, vol. 30, pp. 181-186.

[7] De La Croix, David and Philippe Michel (2002): A theory of economic growth: dynamics and policy in overlapping generations. Cambridge University Press.

[8] Demichelis, S. and H. M. Polemarchakis (2007): "The determinacy of equilibrium in economies of overlapping generations". Economic Theory, vol. 32(3), pp. 3461-475, doi:10.1007/s00199-006-0134-2.

[9] Doob, J. L. (1953): Stochastic Processes. John Wiley \& Sons, Inc., New York.

[10] Erosa, Andrès and Martin Gervais (2002): "Optimal Taxation in Life-Cycle Economies". Journal of Economic Theory, vol. 105, pp. 338-369.

[11] Geanakoplos, John D. and Herakles M. Polemarchakis (1991): "Overlapping Generations". In Handbook of Mathematical Economics, edited by Hildenbrand, Werner and Hugo Sonnenschein, vol. 4, chap. 35, pp. 18991960, Elsevier.

[12] Gel'Fand, Israel M. and Georgeii E. Shilov (1959): Obobshennuye funkzii $i$ deistviya nad nimi. 2nd edn., Fizmatgiz, Moscow.

[13] Jörgens, Konrad (1982): Linear Integral Operators. Pitman Advanced Publishing Program, Boston, London, Melbourne. Translated by G.F. Roach from Lineare Integral operatoren, 1970.

[14] Kenoe, Timothy J. and David K. Levine (March 1985): "Comparative Statics and Perfect Foresight in Infinite Horizon Economies". Econometrica, vol. 53(2), pp. 433-453.

[15] Kotlikoff, Laurence J. (2002): "Generational Policy". In Handbook of Public Economics, edited by Auerbach, Alan J. and Martin Feldstein, vol. 4, chap. 27, pp. 1873-1932, Elsevier.

[16] LAng, Serge (1969): Real analysis. Addison-Wesley.

[17] Mertens, Jean-François and Anna Rubinchik (2009): "Regularity and stability of equilibria in an overlapping generations model with exogenous growth". 
Discussion Paper 2009/5, CORE, Université Catholique de Louvain, Louvainla-Neuve, Belgium. URL http://econ.haifa.ac.il/ arubinchik/papers/ Reg_Stab_OLG.pdf.

[18] - (2012): "Intergenerational equity and the discount rate for policy analysis". Macroeconomic Dynamics, vol. 16, pp. 61-93. URL http://journals. cambridge.org/repo_A84Ar9FF.

[19] - (2013): "Equilibria in an overlapping generations model with transfer policies and exogenous growth". Economic Theory, vol. 54(3), pp. 537-595.

[20] - (2014): "Essential properties of $L_{p, q}$ spaces (the amalgams) and the implicit function theorem for equilibrium analysis in continuous time". Journal of Mathematical Economics. Forthcoming.

[21] Muller, Walter J. III and Michael Woodford (1988): "Determinacy of Equilbrium in Stationary Economies with Both Finite and Infinite Lived Consumers". Journal of Economic Theory, vol. 46, pp. 255-290.

[22] Samuelson, Paul A. (1958): "An Exact Consumption-Loan Model of Interest with or without the Social Contrivance of Money". The Journal of Political Economy, vol. 66(6), pp. 467-482.

[23] Shannon, Chris and William R. Zame (2002): "Quadratic Concavity and Determinacy of Equilibrium". Econometrica, vol. 70(2), pp. 631-662. 